Supporting Information for

\title{
Perfluoroalkylation of Terminal Alkynes with Perfluoroalkyl Iodides Catalyzed by an Iron Salt
}

Wei Li, Changfa Liang, Baogui Luo, Zhenhui Wang, Hengyuan Li, Xiaofeng Li, Huanjian Yang, Huaifeng $\mathrm{Li}^{*}$

State Key Laboratory for Chemistry and Molecular Engineering of Medicinal Resources, School of Chemistry and Pharmaceutical Sciences, Guangxi Normal University, Guilin 541004, China

*E-mail: huaifengli@gxnu.edu.cn 


\section{Table of Contents}

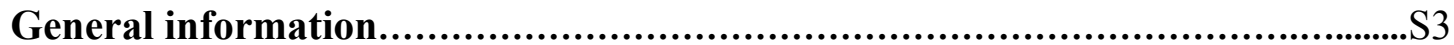

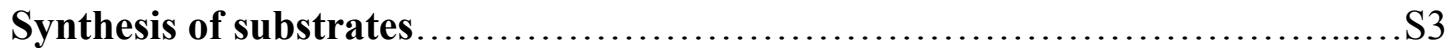

General procedure for iron-catalyzed perfluoroalkylation reaction..............S4

Procedure for the irradiation experiment.................................... 4

Procedure for gram-scale reaction ..................................... 4

Procedure for radical clock experiment..................................... 5

Table S1. Additional optimization data...................................... S5

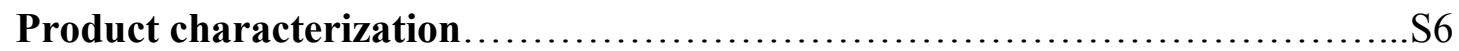

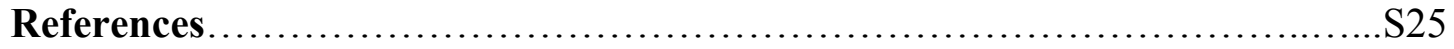

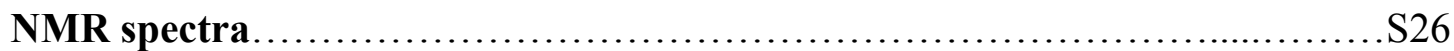

Figure S1. Crude ${ }^{19}$ F NMR of TEMPO-C $\mathbf{F}_{\mathbf{4}} \mathrm{F}_{\mathbf{9}}$ adduct.................................. 67

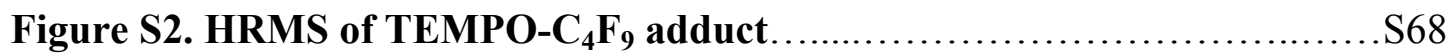




\section{General information}

Unless otherwise noted, all reagents and solvents were obtained commercially and used without further purification. Column chromatography on silica gel (300-400 mesh) was carried out using technical grade $60-90{ }^{\circ} \mathrm{C}$ petroleum ether and analytical grade EtOAc (without further purification). ${ }^{1} \mathrm{H},{ }^{13} \mathrm{C}$ and ${ }^{19} \mathrm{~F}$ NMR spectra were recorded on a $400 \mathrm{MHz}$ or $600 \mathrm{MHz}$ spectrometer. Chemical shifts were reported in ppm. ${ }^{1} \mathrm{H}$ NMR spectra were referenced to $\mathrm{CDCl}_{3}(7.26 \mathrm{ppm})$, and ${ }^{13} \mathrm{C}$ NMR spectra were referenced to $\mathrm{CDCl}_{3}(77.0 \mathrm{ppm})$. Peak multiplicities were designated by the following abbreviations: $\mathrm{s}$, singlet; d, doublet; $\mathrm{t}$, triplet; $\mathrm{m}$, multiplet; brs, broad singlet and $J$, coupling constant in Hz. The HRMS spectrum was measured by micromass QTOF2 Quadrupole/Time of Flight Tandemmass spectrometer with electron spray ionization.

\section{Synthesis of substrates}

All of the alkynes $\mathbf{1}$ (except $\mathbf{1 r}$ and $\mathbf{1} \mathbf{u}$ ) and perfluoroalkylated reagents $\mathbf{2}$ belong to known compounds, which were either purchased from Energy Chemical, Innochem or Sigma-Aldrich.

The unknown $N$-methyl- $N$-phenylhept-6-ynamide $\mathbf{1 r}$ was prepared with the following procedure: A mixture of 6-heptynoic acid (63 mg, $0.5 \mathrm{mmol})$ and oxalyl chloride $(127 \mu \mathrm{L}, 1.5 \mathrm{mmol})$ in dry $\mathrm{CH}_{2} \mathrm{Cl}_{2}(5 \mathrm{~mL})$ was stirred at room temperature for $4 \mathrm{~h}$. Then the solvent and oxalyl chloride were removed under reduced pressure. $\mathrm{N}$ Methylaniline (53.5 mg, $0.5 \mathrm{mmol}), \mathrm{Et}_{3} \mathrm{~N}(139 \mathrm{uL}, 1.0 \mathrm{mmol})$ and dry $\mathrm{CH}_{2} \mathrm{Cl}_{2}(5 \mathrm{~mL})$ were added and the mixture was stirred for $4 \mathrm{~h}$ at room temperature. The reaction mixture was then diluted with $1 \mathrm{M} \mathrm{HCl}(\mathrm{aq})$, washed with brine, dried over $\mathrm{MgSO}_{4}$, filtered and concentrated. The residue was purified with silica gel chromatography (petroleum ether/ethyl ether $=10: 1)$ to provide pure product $(102 \mathrm{mg}, 95 \%)$ as a pale yellow oil.

The unknown Febuxostat-tethered alkyne 1u was prepared with the following procedure: A mixture of Febuxostat $(158 \mathrm{mg}, 0.5 \mathrm{mmol})$ and oxalyl chloride $(127 \mu \mathrm{L}$, $1.5 \mathrm{mmol})$ in dry $\mathrm{CH}_{2} \mathrm{Cl}_{2}(5 \mathrm{~mL})$ was stirred at room temperature for $4 \mathrm{~h}$. Then the solvent and oxalyl chloride were removed under reduced pressure. 4-Ethynylaniline 
(58.6 mg, $0.5 \mathrm{mmol}), \mathrm{Et}_{3} \mathrm{~N}(139 \mathrm{uL})$ and dry $\mathrm{CH}_{2} \mathrm{Cl}_{2}(5 \mathrm{~mL})$ were added and the mixture was stirred for $4 \mathrm{~h}$ at room temperature. The reaction mixture was then diluted with $1 \mathrm{M}$ $\mathrm{HCl}(\mathrm{aq})$, washed with brine, dried over $\mathrm{MgSO}_{4}$, filtered and concentrated. The residue was purified with silica gel chromatography (petroleum ether/ethyl ether $=4: 1$ ) to provide pure product (170 $\mathrm{mg}, 82 \%)$ as a white solid.

\section{General procedure for iron-catalyzed perfluoroalkylation reaction}

Under $\mathrm{N}_{2}$ atmosphere, a $25 \mathrm{~mL}$ sealed tube equipped with a stir bar was charged with 1 (0.2 mmol, 1 equiv) and 2 (0.4 mmol, 2 equiv), $\mathrm{FeCl}_{2} \bullet 4 \mathrm{H}_{2} \mathrm{O}(0.04 \mathrm{mmol}, 8 \mathrm{mg}, 0.2$ equiv), $\mathrm{Cs}_{2} \mathrm{CO}_{3}(0.6 \mathrm{mmol}, 196 \mathrm{mg}, 3$ equiv) and $2 \mathrm{~mL}$ of $t \mathrm{BuOH}$. The reaction mixture was stirred and heated at $70^{\circ} \mathrm{C}$ (oil bath). After 8 hours, the reaction mixture was cooled to room temperature. The resulting mixture was diluted with ethyl acetate and water. Following phase separation, the aqueous layer was extracted with ethyl acetate. The combined organic layers were washed with brine, dried over anhydrous $\mathrm{MgSO}_{4}$, and evaporated under reduced pressure (rotary evaporator). The residue was purified by column chromatography to give the desired product.

\section{Procedure for the irradiation experiment}

A $25 \mathrm{~mL}$ Schlenk flask equipped with a stir bar was charged with $1 \mathrm{a}(0.2 \mathrm{mmol}, 22$ $\mu \mathrm{L}, 1$ equiv) and $2 \mathrm{a}\left(0.4 \mathrm{mmol}, 70 \mu \mathrm{L}, 2\right.$ equiv), $\mathrm{Cs}_{2} \mathrm{CO}_{3}(0.6 \mathrm{mmol}, 196 \mathrm{mg}, 3$ equiv) and $2 \mathrm{~mL}$ of $t \mathrm{BuOH}$. The vessel was evacuated and backfilled with $\mathrm{N}_{2}$ three times. The tube was screw-capped and stirred at $70{ }^{\circ} \mathrm{C}$ (oil bath) under irradiation of $30 \mathrm{~W}$ blue LED (distance app. $5 \mathrm{~cm}$ ) for 8 hours. Then, the reaction mixture was cooled to room temperature. The resulting mixture was diluted and detected by ${ }^{19} \mathrm{~F}$ NMR.

\section{Procedure for gram-scale reaction}

Under $\mathrm{N}_{2}$ atmosphere, a $100 \mathrm{~mL}$ sealed tube equipped with a stir bar was charged with $\mathbf{1 d}(6.3 \mathrm{mmol}, 1.0 \mathrm{~g})$ and $\mathbf{2 a}(12.6 \mathrm{mmol}, 2.2 \mathrm{~mL}), \mathrm{FeCl}_{2} \bullet 4 \mathrm{H}_{2} \mathrm{O}(1.3 \mathrm{mmol}, 249$ $\mathrm{mg}), \mathrm{Cs}_{2} \mathrm{CO}_{3}(18.9 \mathrm{mmol}, 6.2 \mathrm{~g})$ and $60 \mathrm{~mL}$ of $t \mathrm{BuOH}$. The reaction mixture was stirred and heated at $70{ }^{\circ} \mathrm{C}$ (oil bath) for 8 hours, then the reaction mixture was cooled to room 
temperature. The resulting mixture was diluted with ethyl acetate and water. Following phase separation, the aqueous layer was extracted with ethyl acetate. The combined organic layers were washed with brine, dried over anhydrous $\mathrm{MgSO}_{4}$, and evaporated under reduced pressure (rotary evaporator). The residue was purified by column chromatography to afford pure product $\mathbf{3 d}$ as a pale yellow oil $(1.91 \mathrm{~g}, 81 \%)$.

\section{Procedure for radical clock experiment}

Under $\mathrm{N}_{2}$ atmosphere, a $25 \mathrm{~mL}$ sealed tube equipped with a stir bar was charged with 1d $(0.2 \mathrm{mmol}, 32 \mathrm{mg})$ and $2 \mathrm{a}(0.4 \mathrm{mmol}, 70 \mu \mathrm{L}), \mathrm{FeCl}_{2} \bullet 4 \mathrm{H}_{2} \mathrm{O}(0.04 \mathrm{mmol}, 8 \mathrm{mg})$, $\mathrm{Cs}_{2} \mathrm{CO}_{3}(0.6 \mathrm{mmol}, 196 \mathrm{mg})$ and $2 \mathrm{~mL}$ of $t \mathrm{BuOH}$. Subsequently, $\alpha$-cyclopropylstyrene $10(0.2 \mathrm{mmol}, 29 \mathrm{mg})$ was added. The reaction mixture was stirred and heated at $70{ }^{\circ} \mathrm{C}$ (oil bath). After 8 hours, the reaction mixture was cooled to room temperature. The resulting mixture was diluted with ethyl acetate and water. Following phase separation, the aqueous layer was extracted with ethyl acetate. The combined organic layers were washed with brine, dried over anhydrous $\mathrm{MgSO}_{4}$, and evaporated under reduced pressure (rotary evaporator). The residue was purified by column chromatography to afford pure product 11 as a pale yellow oil (27 mg, 37\%).

Table S1. Additional optimization data ${ }^{a}$

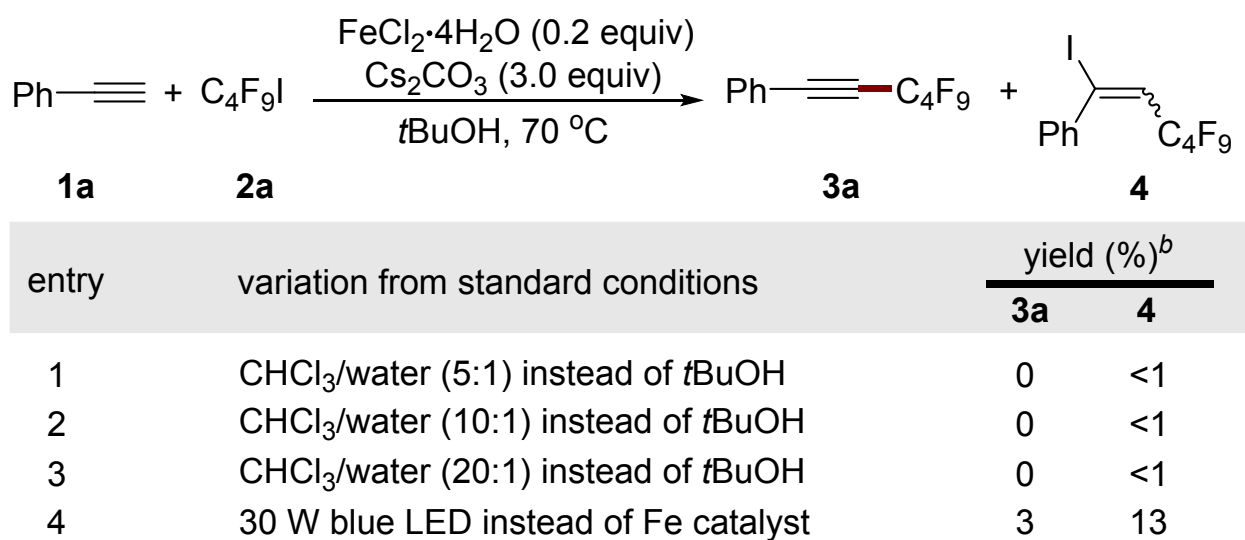

${ }^{a}$ Condition: 1a $(0.2 \mathrm{mmol}), 2 \mathrm{a}(0.4 \mathrm{mmol}), \mathrm{tBuOH}(2.0 \mathrm{~mL}), 70^{\circ} \mathrm{C}, 8 \mathrm{~h}$, under $\mathrm{N}_{2}$. ${ }^{b 19} \mathrm{~F}$ NMR yield with $\mathrm{PhCF}_{3}$ as the internal standard.

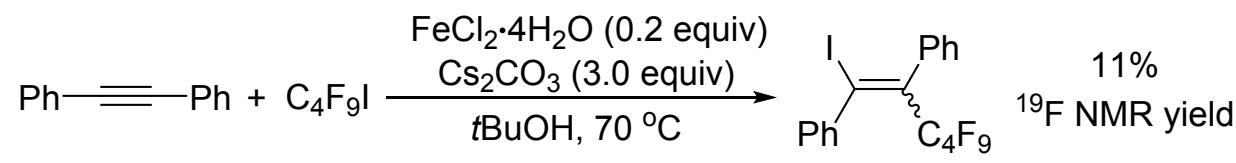




\section{Product characterization}<smiles>FC(F)(F)C#Cc1ccccc1</smiles>

(perfluorohex-1-yn-1-yl)benzene (3a). The general procedure for iron-catalyzed perfluoroalkylation reaction as described previously was followed to synthesize 3a from 1a $(0.2 \mathrm{mmol}, 22 \mu \mathrm{L})$ and $\mathbf{2 a}(0.4 \mathrm{mmol}, 70 \mu \mathrm{L}), \mathrm{FeCl}_{2} \bullet 4 \mathrm{H}_{2} \mathrm{O}(0.04 \mathrm{mmol}, 8 \mathrm{mg})$, $\mathrm{Cs}_{2} \mathrm{CO}_{3}(0.6 \mathrm{mmol}, 196 \mathrm{mg})$ and $2 \mathrm{~mL}$ of $t \mathrm{BuOH}$. The reaction mixture was stirred and heated at $70{ }^{\circ} \mathrm{C}$ (oil bath) for 8 hours, followed by a workup. The residue was purified by flash column chromatography on silica gel (petroleum ether) to afford 3a (49 mg, $76 \%$ yield $)$ as a pale yellow oil. This compound is known. ${ }^{1}{ }^{1} \mathbf{H ~ N M R}\left(600 \mathrm{MHz}, \mathrm{CDCl}_{3}\right)$ $\delta$ 7.50-7.47 (m, 2H), $7.42(\mathrm{t}, J=7.6 \mathrm{~Hz}, 1 \mathrm{H}), 7.33(\mathrm{t}, J=7.8 \mathrm{~Hz}, 2 \mathrm{H}) .{ }^{19}$ F NMR $(565$ $\left.\mathrm{MHz}, \mathrm{CDCl}_{3}\right) \delta-81.0(\mathrm{t}, J=9.6 \mathrm{~Hz}, 3 \mathrm{~F}),-97.4(\mathrm{~m}, 2 \mathrm{~F}),-123.4(\mathrm{~m}, 2 \mathrm{~F}),-125.4(\mathrm{~m}, 2 \mathrm{~F})$.<smiles>FC(F)(F)C#Cc1ccc(-c2ccccc2)cc1</smiles>

4-(perfluorohex-1-yn-1-yl)-1,1'-biphenyl (3b). The general procedure for ironcatalyzed perfluoroalkylation reaction as described previously was followed to synthesize $\mathbf{3 b}$ from $\mathbf{1 b}(0.2 \mathrm{mmol}, 36 \mathrm{mg})$ and $\mathbf{2 a}(0.4 \mathrm{mmol}, 70 \mu \mathrm{L}), \mathrm{FeCl}_{2} \bullet 4 \mathrm{H}_{2} \mathrm{O}(0.04$ mmol, $8 \mathrm{mg}), \mathrm{Cs}_{2} \mathrm{CO}_{3}(0.6 \mathrm{mmol}, 196 \mathrm{mg})$ and $2 \mathrm{~mL}$ of $t \mathrm{BuOH}$. The reaction mixture was stirred and heated at $70{ }^{\circ} \mathrm{C}$ (oil bath) for 8 hours, followed by a workup. The residue was purified by flash column chromatography on silica gel (petroleum ether) to afford 3b (58 mg, 73\% yield) as a white solid. This compound is known. ${ }^{1} \mathbf{H}$ NMR (600 MHz, $\left.\mathrm{CDCl}_{3}\right) \delta 7.54(\mathrm{~s}, 4 \mathrm{H}), 7.50(\mathrm{~d}, J=7.0 \mathrm{~Hz}, 2 \mathrm{H}), 7.38(\mathrm{t}, J=7.7 \mathrm{~Hz}, 2 \mathrm{H}), 7.34-7.28(\mathrm{~m}$, 1H). ${ }^{19}$ F NMR $\left(565 \mathrm{MHz}, \mathrm{CDCl}_{3}\right) \delta-81.0(\mathrm{t}, J=9.5 \mathrm{~Hz}, 3 \mathrm{~F}),-97.3(\mathrm{~m}, 2 \mathrm{~F}),-123.5(\mathrm{~m}$, 2F), -125.4 (m, 2F). 


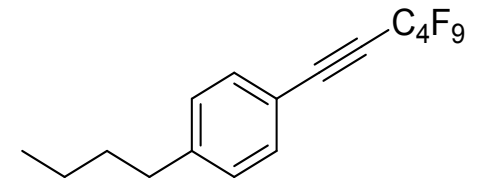

1-(perfluorohex-1-yn-1-yl)-4-butylalkylbenzene (3c). The general procedure for iron-catalyzed perfluoroalkylation reaction as described previously was followed to synthesize 3c from $\mathbf{1 c}(0.2 \mathrm{mmol}, 32 \mathrm{mg})$ and $\mathbf{2 a}(0.4 \mathrm{mmol}, 70 \mu \mathrm{L}), \mathrm{FeCl}_{2} \bullet 4 \mathrm{H}_{2} \mathrm{O}(0.04$ mmol, $8 \mathrm{mg}), \mathrm{Cs}_{2} \mathrm{CO}_{3}(0.6 \mathrm{mmol}, 196 \mathrm{mg})$ and $2 \mathrm{~mL}$ of $t \mathrm{BuOH}$. The reaction mixture was stirred and heated at $70{ }^{\circ} \mathrm{C}$ (oil bath) for 8 hours, followed by a workup. The residue was purified by flash column chromatography on silica gel (petroleum ether) to afford 3c (61 mg, 81\% yield) as a pale yellow oil. ${ }^{1} \mathbf{H}$ NMR $\left(600 \mathrm{MHz}, \mathrm{CDCl}_{3}\right) \delta 7.39(\mathrm{~d}, J=$ $8.1 \mathrm{~Hz}, 2 \mathrm{H}), 7.13(\mathrm{~d}, J=8.2 \mathrm{~Hz}, 2 \mathrm{H}), 2.56(\mathrm{t}, J=7.8 \mathrm{~Hz}, 2 \mathrm{H}), 1.59-1.43(\mathrm{~m}, 2 \mathrm{H}), 1.29-$ $1.23(\mathrm{~m}, 2 \mathrm{H}), 0.85(\mathrm{t}, J=7.4 \mathrm{~Hz}, 3 \mathrm{H}) .{ }^{13} \mathbf{C}\left\{{ }^{1} \mathbf{H}\right\} \mathbf{N M R}\left(151 \mathrm{MHz}, \mathrm{CDCl}_{3}\right) \delta 146.7,132.5$ (t, $J=2.6 \mathrm{~Hz}), 128.8,118.4-116.4$ [m, $\left.-\left(\mathrm{CF}_{2}\right) \mathrm{CF}_{3}\right], 115.6(\mathrm{t}, J=3.1 \mathrm{~Hz}), 114.7-105.7$ [m, $\left.-\left(\mathrm{CF}_{2}\right) \mathrm{CF}_{3}\right], 92.8(\mathrm{t}, J=6.4 \mathrm{~Hz}), 74.1(\mathrm{t}, J=36.0 \mathrm{~Hz}), 35.8,33.2,22.3,13.9 .{ }^{19} \mathbf{F}$ NMR $\left(565 \mathrm{MHz}, \mathrm{CDCl}_{3}\right) \delta-81.0(\mathrm{t}, J=9.7 \mathrm{~Hz}, 3 \mathrm{~F}),-97.1(\mathrm{t}, J=9.1 \mathrm{~Hz}, 2 \mathrm{~F}),-123.4$ (m, 2F), -125.5 (m, 2F). HRMS (EI) m/z: [M] $]^{+}$Calcd for $\mathrm{C}_{16} \mathrm{H}_{13} \mathrm{~F}_{9}{ }^{+}: 376.0868$; Found 376.0875 .

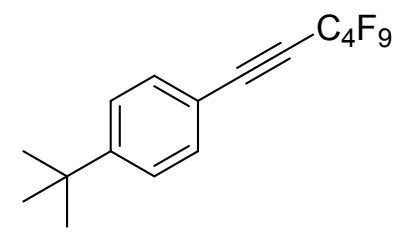

1-(perfluorohex-1-yn-1-yl)-4-tert-butylkylbenzene (3d). The general procedure for iron-catalyzed perfluoroalkylation reaction as described previously was followed to synthesize 3d from $\mathbf{1 d}(0.2 \mathrm{mmol}, 32 \mathrm{mg})$ and $\mathbf{2 a}(0.4 \mathrm{mmol}, 70 \mu \mathrm{L}), \mathrm{FeCl}_{2} \bullet 4 \mathrm{H}_{2} \mathrm{O}(0.04$ mmol, $8 \mathrm{mg}), \mathrm{Cs}_{2} \mathrm{CO}_{3}(0.6 \mathrm{mmol}, 196 \mathrm{mg})$ and $2 \mathrm{~mL}$ of $t \mathrm{BuOH}$. The reaction mixture was stirred and heated at $70{ }^{\circ} \mathrm{C}$ (oil bath) for 8 hours, followed by a workup. The residue was purified by flash column chromatography on silica gel (petroleum ether) to afford 3d (63 mg, 84\% yield) as a pale yellow oil. This compound is known. ${ }^{1} \mathbf{H}$ NMR (600 $\left.\mathrm{MHz}, \mathrm{CDCl}_{3}\right) \delta 7.42(\mathrm{~d}, J=8.4 \mathrm{~Hz}, 2 \mathrm{H}), 7.34(\mathrm{~d}, J=8.5 \mathrm{~Hz}, 2 \mathrm{H}), 1.24(\mathrm{~s}, 9 \mathrm{H}) .{ }^{19} \mathbf{F}$ NMR $\left(565 \mathrm{MHz}, \mathrm{CDCl}_{3}\right) \delta-81.0(\mathrm{t}, J=9.6 \mathrm{~Hz}, 3 \mathrm{~F}),-97.1(\mathrm{~m}, 2 \mathrm{~F}),-123.4(\mathrm{~m}, 2 \mathrm{~F}),-$ 


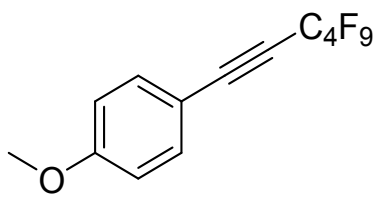

1-methoxy-4-(perfluorohex-1-yn-1-yl)benzene (3e). The general procedure for ironcatalyzed perfluoroalkylation reaction as described previously was followed to synthesize 3e from $\mathbf{1 e}(0.2 \mathrm{mmol}, 26 \mathrm{mg})$ and $\mathbf{2 a}(0.4 \mathrm{mmol}, 70 \mu \mathrm{L}), \mathrm{FeCl}_{2} \bullet 4 \mathrm{H}_{2} \mathrm{O}(0.04$ mmol, $8 \mathrm{mg}), \mathrm{Cs}_{2} \mathrm{CO}_{3}(0.6 \mathrm{mmol}, 196 \mathrm{mg})$ and $2 \mathrm{~mL}$ of $t \mathrm{BuOH}$. The reaction mixture was stirred and heated at $70{ }^{\circ} \mathrm{C}$ (oil bath) for 8 hours, followed by a workup. The residue was purified by flash column chromatography on silica gel (petroleum ether) to afford 3e (60 mg, 86\% yield) as a pale yellow oil. This compound is known. ${ }^{1} \mathbf{H}$ NMR (600 $\left.\mathrm{MHz}, \mathrm{CDCl}_{3}\right) \delta 7.41(\mathrm{~d}, J=8.8 \mathrm{~Hz}, 2 \mathrm{H}), 6.81(\mathrm{~d}, J=8.8 \mathrm{~Hz}, 2 \mathrm{H}), 3.76(\mathrm{~s}, 3 \mathrm{H}) .{ }^{19} \mathbf{F}$ NMR $\left(565 \mathrm{MHz}, \mathrm{CDCl}_{3}\right) \delta-81.1(\mathrm{t}, J=9.7 \mathrm{~Hz}, 3 \mathrm{~F}),-96.8(\mathrm{t}, J=9.1 \mathrm{~Hz}, 2 \mathrm{~F}),-123.4$ $(\mathrm{m}, 2 \mathrm{~F}),-125.5(\mathrm{~m}, 2 \mathrm{~F})$.<smiles>Fc1ccc(C#CC(F)(F)F)cc1</smiles>

1-fluoro-4-(perfluorohex-1-yn-1-yl)benzene (3f). The general procedure for ironcatalyzed perfluoroalkylation reaction as described previously was followed to synthesize 3f from $\mathbf{1 f}(0.2 \mathrm{mmol}, 24 \mathrm{mg})$ and $\mathbf{2 a}(0.4 \mathrm{mmol}, 70 \mu \mathrm{L}), \mathrm{FeCl}_{2} \bullet 4 \mathrm{H}_{2} \mathrm{O}(0.04$ mmol, $8 \mathrm{mg}), \mathrm{Cs}_{2} \mathrm{CO}_{3}(0.6 \mathrm{mmol}, 196 \mathrm{mg})$ and $2 \mathrm{~mL}$ of $t \mathrm{BuOH}$. The reaction mixture was stirred and heated at $70{ }^{\circ} \mathrm{C}$ (oil bath) for 14 hours, followed by a workup. The residue was purified by flash column chromatography on silica gel (petroleum ether) to afford $3 \mathrm{f}$ (38 mg, 56\% yield) as a pale yellow oil. This compound is known. ${ }^{1}{ }^{1} \mathbf{H}$ NMR $\left(600 \mathrm{MHz}, \mathrm{CDCl}_{3}\right) \delta$ 7.51-7.48 (m, 2H), $7.04(\mathrm{t}, J=8.6 \mathrm{~Hz}, 2 \mathrm{H}) .{ }^{19} \mathbf{F}$ NMR $(565 \mathrm{MHz}$, $\left.\mathrm{CDCl}_{3}\right) \delta-81.0(\mathrm{t}, J=9.5 \mathrm{~Hz}, 3 \mathrm{~F}),-97.5(\mathrm{t}, J=8.9 \mathrm{~Hz}, 2 \mathrm{~F}),-105.5(\mathrm{~s}, 1 \mathrm{~F}),-123.4(\mathrm{~m}$, $2 \mathrm{~F}),-125.4(\mathrm{~m}, 2 \mathrm{~F})$. 
(1)

1-amido-4-(perfluorohex-1-yn-1-yl)benzene (3g). The general procedure for ironcatalyzed perfluoroalkylation reaction as described previously was followed to synthesize $\mathbf{3 g}$ from $\mathbf{1 g}(0.2 \mathrm{mmol}, 23 \mathrm{mg})$ and $\mathbf{2 a}(0.4 \mathrm{mmol}, 70 \mu \mathrm{L}), \mathrm{FeCl}_{2} \bullet 4 \mathrm{H}_{2} \mathrm{O}(0.04$ mmol, $8 \mathrm{mg}), \mathrm{Cs}_{2} \mathrm{CO}_{3}(0.6 \mathrm{mmol}, 196 \mathrm{mg})$ and $2 \mathrm{~mL}$ of $t \mathrm{BuOH}$. The reaction mixture was stirred and heated at $70{ }^{\circ} \mathrm{C}$ (oil bath) for 8 hours, followed by a workup. The residue was purified by flash column chromatography on silica gel (petroleum ether/ethyl ether $=40: 1)$ to afford $\mathbf{3 g}\left(42 \mathrm{mg}, 62 \%\right.$ yield) as a pale yellow oil. This compound is known. ${ }^{2}$ ${ }^{1} \mathbf{H}$ NMR $\left(600 \mathrm{MHz}, \mathrm{CDCl}_{3}\right) \delta 7.27(\mathrm{~d}, J=8.5 \mathrm{~Hz}, 2 \mathrm{H}), 6.54(\mathrm{~d}, J=8.6 \mathrm{~Hz}, 2 \mathrm{H}), 3.93$ (s, 2H). ${ }^{19}$ F NMR $\left(565 \mathrm{MHz}, \mathrm{CDCl}_{3}\right) \delta-81.0(\mathrm{t}, J=9.8 \mathrm{~Hz}, 3 \mathrm{~F}),-96.2(\mathrm{t}, J=8.8 \mathrm{~Hz}$, 2F), -123.4 (m, 2F), -125.4 (m, 2F).

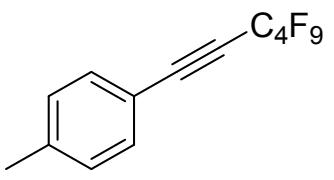

1-methyl-4-(perfluorohex-1-yn-1-yl)benzene (3h). The general procedure for ironcatalyzed perfluoroalkylation reaction as described previously was followed to synthesize $3 \mathbf{h}$ from $\mathbf{1 h}(0.2 \mathrm{mmol}, 23 \mathrm{mg})$ and $\mathbf{2 a}(0.4 \mathrm{mmol}, 70 \mu \mathrm{L}), \mathrm{FeCl}_{2} \bullet 4 \mathrm{H}_{2} \mathrm{O}(0.04$ mmol, $8 \mathrm{mg}), \mathrm{Cs}_{2} \mathrm{CO}_{3}(0.6 \mathrm{mmol}, 196 \mathrm{mg})$ and $2 \mathrm{~mL}$ of $t \mathrm{BuOH}$. The reaction mixture was stirred and heated at $70{ }^{\circ} \mathrm{C}$ (oil bath) for 8 hours, followed by a workup. The residue was purified by flash column chromatography on silica gel (petroleum ether) to afford 3h (50 mg, 75\% yield) as a pale yellow oil. This compound is known. ${ }^{3} \mathbf{1}$ H NMR (600 $\left.\mathrm{MHz}, \mathrm{CDCl}_{3}\right) \delta 7.38(\mathrm{~d}, J=7.9 \mathrm{~Hz}, 2 \mathrm{H}), 7.13(\mathrm{~d}, J=7.8 \mathrm{~Hz}, 2 \mathrm{H}), 2.32(\mathrm{~s}, 3 \mathrm{H}) .{ }^{19} \mathbf{F}$ NMR $\left(565 \mathrm{MHz}, \mathrm{CDCl}_{3}\right) \delta-81.0(\mathrm{t}, J=9.6 \mathrm{~Hz}, 3 \mathrm{~F}),-97.1(\mathrm{~m}, 2 \mathrm{~F}),-123.4(\mathrm{~m}, 2 \mathrm{~F}),-$ $125.5(\mathrm{~m}, 2 \mathrm{~F})$ 
(c)

1-chloro-4-(perfluorohex-1-yn-1-yl)benzene (3i). The general procedure for ironcatalyzed perfluoroalkylation reaction as described previously was followed to synthesize 3i from $\mathbf{1 i}(0.2 \mathrm{mmol}, 27 \mathrm{mg})$ and $\mathbf{2 a}(0.4 \mathrm{mmol}, 70 \mu \mathrm{L}), \mathrm{FeCl}_{2} \bullet 4 \mathrm{H}_{2} \mathrm{O}(0.04$ mmol, $8 \mathrm{mg}), \mathrm{Cs}_{2} \mathrm{CO}_{3}(0.6 \mathrm{mmol}, 196 \mathrm{mg})$ and $2 \mathrm{~mL}$ of $t \mathrm{BuOH}$. The reaction mixture was stirred and heated at $70{ }^{\circ} \mathrm{C}$ (oil bath) for 8 hours, followed by a workup. The residue was purified by flash column chromatography on silica gel (petroleum ether) to afford $3 \mathbf{i}\left(42 \mathrm{mg}, 60 \%\right.$ yield) as a pale yellow oil. ${ }^{1} \mathbf{H}$ NMR $\left(600 \mathrm{MHz}, \mathrm{CDCl}_{3}\right) \delta 7.43(\mathrm{~d}, J=$ $8.3 \mathrm{~Hz}, 2 \mathrm{H}), 7.32(\mathrm{~d}, J=8.4 \mathrm{~Hz}, 2 \mathrm{H}) .{ }^{19} \mathbf{F} \mathbf{N M R}\left(565 \mathrm{MHz}, \mathrm{CDCl}_{3}\right) \delta-81.0(\mathrm{t}, J=9.6$ $\mathrm{Hz}, 3 \mathrm{~F}),-97.7$ (m, 2F), -123.4 (m, 2F), -125.4 (m, 2F).<smiles>Nc1ccccc1C#CC(F)(F)F</smiles>

1-amido-2-(perfluorohex-1-yn-1-yl)benzene (3l). The general procedure for ironcatalyzed perfluoroalkylation reaction as described previously was followed to synthesize 31 from 11 (0.2 mmol, $23 \mathrm{mg})$ and $\mathbf{2 a}(0.4 \mathrm{mmol}, 70 \mu \mathrm{L}), \mathrm{FeCl}_{2} \bullet 4 \mathrm{H}_{2} \mathrm{O}(0.04$ mmol, $8 \mathrm{mg}), \mathrm{Cs}_{2} \mathrm{CO}_{3}(0.6 \mathrm{mmol}, 196 \mathrm{mg})$ and $2 \mathrm{~mL}$ of $t \mathrm{BuOH}$. The reaction mixture was stirred and heated at $70{ }^{\circ} \mathrm{C}$ (oil bath) for 8 hours, followed by a workup. The residue was purified by flash column chromatography on silica gel (petroleum ether/ethyl ether $=40: 1)$ to afford $\mathbf{3 l}$ ( $47 \mathrm{mg}, 70 \%$ yield) as a pale yellow oil. This compound is known. ${ }^{2}$ ${ }^{1}$ H NMR $\left(600 \mathrm{MHz} \mathrm{CDCl}_{3}\right) \delta$ 7.30-7.24 (m, 1H), 7.20-7.14 (m, 1H), $6.64(\mathrm{t}, J=7.3$ $\mathrm{Hz}, 2 \mathrm{H}), 4.18(\mathrm{~s}, 2 \mathrm{H}) .{ }^{19} \mathbf{F}$ NMR $\left(565 \mathrm{MHz}, \mathrm{CDCl}_{3}\right) \delta-81.0(\mathrm{t}, J=9.7 \mathrm{~Hz}, 3 \mathrm{~F}),-96.5$ $(\mathrm{m}, 2 \mathrm{~F}),-123.1(\mathrm{~m}, 2 \mathrm{~F}),-125.3(\mathrm{~m}, 2 \mathrm{~F})$.<smiles>FC(F)(F)C#Cc1ccccc1Cl</smiles>

1-chloro-2-(perfluorohex-1-yn-1-yl)benzene (3m). The general procedure for iron- 
catalyzed perfluoroalkylation reaction as described previously was followed to synthesize $\mathbf{3 m}$ from $\mathbf{1 m}(0.2 \mathrm{mmol}, 27 \mathrm{mg})$ and $\mathbf{2 a}(0.4 \mathrm{mmol}, 70 \mu \mathrm{L}), \mathrm{FeCl}_{2} \bullet 4 \mathrm{H}_{2} \mathrm{O}$ (0.04 mmol, $8 \mathrm{mg}), \mathrm{Cs}_{2} \mathrm{CO}_{3}(0.6 \mathrm{mmol}, 196 \mathrm{mg})$ and $2 \mathrm{~mL}$ of $t \mathrm{BuOH}$. The reaction mixture was stirred and heated at $70{ }^{\circ} \mathrm{C}$ (oil bath) for 8 hours, followed by a workup. The residue was purified by flash column chromatography on silica gel (petroleum ether) to afford $\mathbf{3 m}$ (36 mg, 51\% yield) as a pale yellow oil. This compound is known. ${ }^{3}$ ${ }^{1} \mathbf{H}$ NMR $\left(600 \mathrm{MHz}, \mathrm{CDCl}_{3}\right) \delta 7.51(\mathrm{dd}, J=7.8,1.6 \mathrm{~Hz}, 1 \mathrm{H}), 7.40(\mathrm{dd}, J=8.2,1.3 \mathrm{~Hz}$, $1 \mathrm{H}), 7.35(\mathrm{td}, J=7.8,1.6 \mathrm{~Hz}, 1 \mathrm{H}), 7.23(\mathrm{td}, J=7.6,1.3 \mathrm{~Hz}, 1 \mathrm{H}) .{ }^{19} \mathbf{F}$ NMR $(565 \mathrm{MHz}$, $\left.\mathrm{CDCl}_{3}\right) \delta-81.0(\mathrm{t}, J=9.5 \mathrm{~Hz}, 3 \mathrm{~F}),-97.9(\mathrm{~m}, 2 \mathrm{~F}),-123.2(\mathrm{~m}, 2 \mathrm{~F}),-125.3(\mathrm{~m}, 2 \mathrm{~F})$.

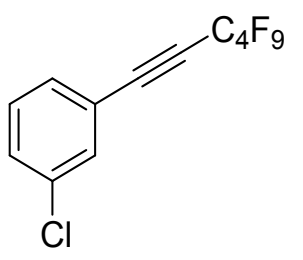

1-chloro-4-(perfluorohex-1-yn-1-yl)benzene (3n). The general procedure for ironcatalyzed perfluoroalkylation reaction as described previously was followed to synthesize 3n from $\mathbf{1 n}(0.2 \mathrm{mmol}, 27 \mathrm{mg})$ and $\mathbf{2 a}(0.4 \mathrm{mmol}, 70 \mu \mathrm{L}), \mathrm{FeCl}_{2} \bullet 4 \mathrm{H}_{2} \mathrm{O}(0.04$ mmol, $8 \mathrm{mg}), \mathrm{Cs}_{2} \mathrm{CO}_{3}(0.6 \mathrm{mmol}, 196 \mathrm{mg})$ and $2 \mathrm{~mL}$ of $t \mathrm{BuOH}$. The reaction mixture was stirred and heated at $70^{\circ} \mathrm{C}$ (oil bath) for 8 hours, followed by a workup. The residue was purified by flash column chromatography on silica gel (petroleum ether) to afford 3n (45 mg, 63\% yield) as a pale yellow oil. ${ }^{1} \mathbf{H}$ NMR $\left(600 \mathrm{MHz}, \mathrm{CDCl}_{3}\right) \delta 7.48(\mathrm{t}, J=$ $1.7 \mathrm{~Hz}, 1 \mathrm{H}), 7.41-7.37(\mathrm{~m}, 2 \mathrm{H}), 7.28(\mathrm{t}, J=7.9 \mathrm{~Hz}, 1 \mathrm{H}) .{ }^{13} \mathbf{C}\left\{{ }^{1} \mathbf{H}\right\}$ NMR $(151 \mathrm{MHz}$, $\left.\mathrm{CDCl}_{3}\right) \delta 134.7,132.3(\mathrm{t}, J=2.5 \mathrm{~Hz}), 131.5,130.6(\mathrm{t}, J=2.7 \mathrm{~Hz}), 130.0,120.1(\mathrm{t}, J=$ $3.2 \mathrm{~Hz}), 118.5-116.4\left[\mathrm{~m},-\left(\mathrm{CF}_{2}\right) \mathrm{CF}_{3}\right], 111.5-105.5\left[\mathrm{~m},-\left(\mathrm{CF}_{2}\right) \mathrm{CF}_{3}\right], 90.5(\mathrm{t}, J=6.3 \mathrm{~Hz})$, $75.5(\mathrm{t}, J=36.4 \mathrm{~Hz}) .{ }^{19} \mathbf{F}$ NMR $\left(565 \mathrm{MHz}, \mathrm{CDCl}_{3}\right) \delta-81.0(\mathrm{t}, J=9.9 \mathrm{~Hz}, 3 \mathrm{~F}),-97.9(\mathrm{~m}$, 2F), $-123.4(\mathrm{~m}, 2 \mathrm{~F}),-125.4(\mathrm{~m}, 2 \mathrm{~F})$. HRMS (ESI) m/z: $[\mathrm{M}+\mathrm{H}]^{+}$Calcd for $\mathrm{C}_{12} \mathrm{H}_{5} \mathrm{ClF}_{9}{ }^{+}$: 354.9931; Found 354.9938. 
<smiles>COc1cc(C#CC(F)(F)F)cc(OC)c1</smiles>

1,3-dimethoxy-5-(perfluorohex-1-yn-1-yl)benzene (3o). The general procedure for iron-catalyzed perfluoroalkylation reaction as described previously was followed to synthesize $3 \mathbf{o}$ from $1 \mathbf{0}(0.2 \mathrm{mmol}, 32 \mathrm{mg})$ and $\mathbf{2 a}(0.4 \mathrm{mmol}, 70 \mu \mathrm{L}), \mathrm{FeCl}_{2} \bullet 4 \mathrm{H}_{2} \mathrm{O}(0.04$ mmol, $8 \mathrm{mg}), \mathrm{Cs}_{2} \mathrm{CO}_{3}(0.6 \mathrm{mmol}, 196 \mathrm{mg})$ and $2 \mathrm{~mL}$ of $t \mathrm{BuOH}$. The reaction mixture was stirred and heated at $70{ }^{\circ} \mathrm{C}$ (oil bath) for 8 hours, followed by a workup. The residue was purified by flash column chromatography on silica gel (petroleum ether/ethyl ether $=40: 1)$ to afford $3 \mathbf{0}\left(46 \mathrm{mg}, 61 \%\right.$ yield) as a pale yellow oil. This compound is known. ${ }^{1}$ ${ }^{1} \mathbf{H}$ NMR $\left(600 \mathrm{MHz}, \mathrm{CDCl}_{3}\right) \delta 6.61(\mathrm{~d}, J=2.3 \mathrm{~Hz}, 2 \mathrm{H}), 6.50(\mathrm{t}, J=2.3 \mathrm{~Hz}, 1 \mathrm{H}), 3.73$ $(\mathrm{s}, 6 \mathrm{H}) .{ }^{19} \mathbf{F}$ NMR $\left(565 \mathrm{MHz}, \mathrm{CDCl}_{3}\right) \delta-81.0(\mathrm{t}, J=9.7 \mathrm{~Hz}, 3 \mathrm{~F}),-97.4(\mathrm{t}, J=8.3 \mathrm{~Hz}$, 2F), $-123.3(\mathrm{~m}, 2 \mathrm{~F}),-125.4(\mathrm{~m}, 2 \mathrm{~F})$.

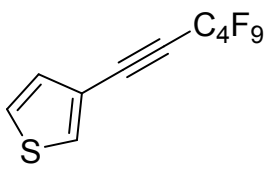

3-(perfluorohex-1-yn-1-yl)thiophene (3p). The general procedure for iron-catalyzed perfluoroalkylation reaction as described previously was followed to synthesize $\mathbf{3 p}$ from $1 \mathbf{p}(0.2 \mathrm{mmol}, 22 \mathrm{mg})$ and $\mathbf{2 a}(0.4 \mathrm{mmol}, 70 \mu \mathrm{L}), \mathrm{FeCl}_{2} \bullet 4 \mathrm{H}_{2} \mathrm{O}(0.04 \mathrm{mmol}, 8 \mathrm{mg})$, $\mathrm{Cs}_{2} \mathrm{CO}_{3}(0.6 \mathrm{mmol}, 196 \mathrm{mg})$ and $2 \mathrm{~mL}$ of $t \mathrm{BuOH}$. The reaction mixture was stirred and heated at $70{ }^{\circ} \mathrm{C}$ (oil bath) for 8 hours, followed by a workup. The residue was purified by flash column chromatography on silica gel (petroleum ether) to afford $\mathbf{3 p}$ (35 mg, $54 \%$ yield $)$ as a pale yellow oil. This compound is known. ${ }^{1}{ }^{1} \mathbf{H}$ NMR $\left(600 \mathrm{MHz}, \mathrm{CDCl}_{3}\right)$ $\delta$ 7.73-7.63 (m, 1H), $7.29(\mathrm{dd}, J=5.0,3.0 \mathrm{~Hz}, 1 \mathrm{H}), 7.15(\mathrm{dd}, J=5.1,1.2 \mathrm{~Hz}, 1 \mathrm{H}) .{ }^{19} \mathbf{F}$ NMR $\left(565 \mathrm{MHz}, \mathrm{CDCl}_{3}\right) \delta-81.0(\mathrm{t}, J=9.7 \mathrm{~Hz}, 3 \mathrm{~F}),-97.2(\mathrm{t}, J=9.5 \mathrm{~Hz}, 2 \mathrm{~F}),-123.3$ $(\mathrm{m}, 2 \mathrm{~F}),-125.4(\mathrm{~m}, 2 \mathrm{~F})$ 


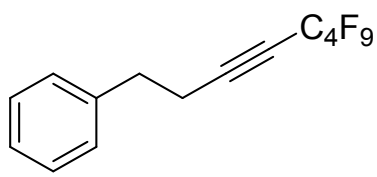

$(1,1,1,2,2,3,3,4,4-n o n a f l u o r o)$-4-phenyl-1-butyne (3q). The general procedure for iron-catalyzed perfluoroalkylation reaction as described previously was followed to synthesize 3q from 1q $(0.2 \mathrm{mmol}, 26 \mathrm{mg})$ and $\mathbf{2 a}(0.4 \mathrm{mmol}, 70 \mu \mathrm{L}), \mathrm{FeCl}_{2} \bullet 4 \mathrm{H}_{2} \mathrm{O}(0.04$ mmol, $8 \mathrm{mg}), \mathrm{Cs}_{2} \mathrm{CO}_{3}(0.6 \mathrm{mmol}, 196 \mathrm{mg})$ and $2 \mathrm{~mL}$ of $t \mathrm{BuOH}$. The reaction mixture was stirred and heated at $70{ }^{\circ} \mathrm{C}$ (oil bath) for 14 hours, followed by a workup. The residue was purified by flash column chromatography on silica gel (petroleum ether) to afford 3q (14 mg, 20\% yield) as a pale yellow oil. ${ }^{1} \mathbf{H}$ NMR $\left(600 \mathrm{MHz}, \mathrm{CDCl}_{3}\right) \delta 7.32$ (t, $J=7.5 \mathrm{~Hz}, 2 \mathrm{H}), 7.26-7.23(\mathrm{~m}, 1 \mathrm{H}), 7.21(\mathrm{~d}, J=6.8 \mathrm{~Hz}, 2 \mathrm{H}), 2.90(\mathrm{t}, J=7.5 \mathrm{~Hz}, 2 \mathrm{H})$, 2.67-2.63 (m, 2H). ${ }^{19} \mathbf{F}$ NMR $\left(565 \mathrm{MHz}, \mathrm{CDCl}_{3}\right) \delta-81.0(\mathrm{t}, J=9.6 \mathrm{~Hz}, 3 \mathrm{~F}),-96.7(\mathrm{~m}$, 2F), -123.4 (m, 2F), -125.5 (m, 2F). ${ }^{13} \mathbf{C}\left\{{ }^{1} \mathbf{H}\right\}$ NMR (151 MHz, $\left.\mathrm{CDCl}_{3}\right)$ 8139.2, 128.8, 128.5, 126.9, 118.4-115.2 [m, $\left.-\left(\mathrm{CF}_{2}\right) \mathrm{CF}_{3}\right], 109.5-106.7\left[\mathrm{~m},-\left(\mathrm{CF}_{2}\right) \mathrm{CF}_{3}\right], 94.4$ (t, $J=6.1$ Hz), $68.1(\mathrm{t}, J=35.8 \mathrm{~Hz}), 33.5(\mathrm{t}, J=2.3 \mathrm{~Hz}), 20.8(\mathrm{t}, J=2.5 \mathrm{~Hz})$. HRMS (EI) m/z: $[\mathrm{M}]^{+}$Calcd for $\mathrm{C}_{14} \mathrm{H}_{9} \mathrm{~F}_{9}{ }^{+}:$348.0555; Found 348.0567.<smiles>C#CC(C)C(=O)N(C)c1ccccc1</smiles>

$N$-methyl- $N$-phenylhept-6-ynamide (1r). The general procedure for the synthesis of substrate 1r as described previously was followed to synthesize it. The product (102 mg, 95\% yield) as a pale yellow oil was purified with silica gel chromatography (petroleum ether/ethyl ether = 10:1). ${ }^{1} \mathbf{H}$ NMR $\left(600 \mathrm{MHz}, \mathrm{CDCl}_{3}\right) \delta 7.42(\mathrm{t}, J=7.7 \mathrm{~Hz}$, 2H), 7.35 (t, $J=7.4 \mathrm{~Hz}, 1 \mathrm{H}), 7.22-7.13$ (m, 2H), 3.26 (s, 3H), 2.11-2.08 (m, 4H), 1.90 $(\mathrm{t}, J=2.6 \mathrm{~Hz}, 1 \mathrm{H}), 1.71-1.66(\mathrm{~m}, 2 \mathrm{H}), 1.55-1.38(\mathrm{~m}, 2 \mathrm{H}) .{ }^{13} \mathbf{C}\left\{{ }^{1} \mathbf{H}\right\}$ NMR $(151 \mathrm{MHz}$, $\left.\mathrm{CDCl}_{3}\right) \delta 172.8,144.1,129.8,127.8,127.3,84.2,68.3,37.3,33.5,28.0,24.6,18.2$. HRMS (ESI) m/z: $[\mathrm{M}+\mathrm{Na}]^{+}$Calcd for $\mathrm{C}_{14} \mathrm{H}_{17} \mathrm{NNaO}^{+}(\mathrm{M}+\mathrm{Na})^{+}$: 238.1208; Found 238.1202 . 
<smiles>CC(C#CC(F)(F)F)C(=O)N(C)c1ccccc1</smiles>

8,8,9,9,10,10,11,11,11-nonafluoro- $N$-methyl- $N$-phenylundec-6-ynamide (3r). The general procedure for iron-catalyzed perfluoroalkylation reaction as described previously was followed to synthesize $3 \mathbf{r}$ from $1 \mathbf{r}(0.2 \mathrm{mmol}, 43 \mathrm{mg})$ and $\mathbf{2 a}(0.4 \mathrm{mmol}$, $70 \mu \mathrm{L}), \mathrm{FeCl}_{2} \bullet 4 \mathrm{H}_{2} \mathrm{O}(0.04 \mathrm{mmol}, 8 \mathrm{mg}), \mathrm{Cs}_{2} \mathrm{CO}_{3}(0.6 \mathrm{mmol}, 196 \mathrm{mg})$ and $2 \mathrm{~mL}$ of $t \mathrm{BuOH}$. The reaction mixture was stirred and heated at $70{ }^{\circ} \mathrm{C}$ (oil bath) for 8 hours, followed by a workup. The residue was purified by flash column chromatography on silica gel (petroleum ether/ethyl ether $=10: 1)$ to afford $3 \mathbf{r}(22 \mathrm{mg}, 25 \%$ yield $)$ as a pale yellow oil. ${ }^{1} \mathbf{H}$ NMR $\left(400 \mathrm{MHz}, \mathrm{CDCl}_{3}\right) \delta$ 7.45-7.35 (m, 2H), 7.37-7.27 (m, 1H), 7.19$7.16(\mathrm{~m}, 2 \mathrm{H}), 3.27(\mathrm{~s}, 3 \mathrm{H}), 2.29(\mathrm{~m}, 2 \mathrm{H}), 2.10(\mathrm{t}, J=7.3 \mathrm{~Hz}, 2 \mathrm{H}), 1.71-1.64(\mathrm{~m}, 2 \mathrm{H})$, 1.56-1.49 (m, 2H). ${ }^{19} \mathbf{F}$ NMR $\left(565 \mathrm{MHz}, \mathrm{CDCl}_{3}\right) \delta-81.0(\mathrm{t}, J=9.6 \mathrm{~Hz}, 3 \mathrm{~F}),-96.5(\mathrm{~m}$, 2F), -123.4 (m, 2F), -125.5 (m, 2F). ${ }^{13} \mathbf{C}\left\{{ }^{1} \mathbf{H}\right\}$ NMR (151 MHz, $\left.\mathrm{CDCl}_{3}\right) \delta 172.5,144.1$, 130.0, 128.0, 127.4, 118.6-116.3 [m, $\left.-\left(\mathrm{CF}_{2}\right) \mathrm{CF}_{3}\right], 110.3-105.3\left[\mathrm{~m},-\left(\mathrm{CF}_{2}\right) \mathrm{CF}_{3}\right], 95.0(\mathrm{t}$, $J=6.2 \mathrm{~Hz}), 67.4(\mathrm{t}, J=35.8 \mathrm{~Hz}), 37.5,33.5,27.0(\mathrm{t}, J=2.3 \mathrm{~Hz}), 24.6,18.5(\mathrm{t}, J=2.6$ Hz). HRMS (ESI) m/z: $[\mathrm{M}+\mathrm{H}]^{+}$Calcd for $\mathrm{C}_{18} \mathrm{H}_{17} \mathrm{~F}_{9} \mathrm{NO}^{+}: 434.1161$; found 434.1157 .

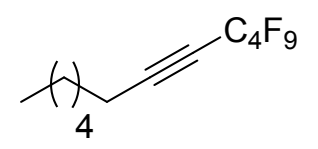

1,1,1,2,2,3,3,4,4-nonafluorododec-5-yne (3s). The general procedure for ironcatalyzed perfluoroalkylation reaction as described previously was followed to synthesize 3s from $\mathbf{1 s}(0.2 \mathrm{mmol}, 22 \mathrm{mg})$ and $\mathbf{2 a}(0.4 \mathrm{mmol}, 70 \mu \mathrm{L}), \mathrm{FeCl}_{2} \cdot 4 \mathrm{H}_{2} \mathrm{O}(0.04$ mmol, $8 \mathrm{mg}), \mathrm{Cs}_{2} \mathrm{CO}_{3}(0.6 \mathrm{mmol}, 196 \mathrm{mg})$ and $2 \mathrm{~mL}$ of $t \mathrm{BuOH}$. The reaction mixture was stirred and heated at $70{ }^{\circ} \mathrm{C}$ (oil bath) for 14 hours, followed by a workup. The residue was purified by flash column chromatography on silica gel (petroleum ether) to afford 3s (18 mg, 27\% yield) as a pale yellow oil. This compound is known. ${ }^{4} \mathbf{H}$ NMR $\left(600 \mathrm{MHz}, \mathrm{CDCl}_{3}\right) \delta$ 2.37-2.33 (m, 2H), 1.61-1.56 (m, 2H), 1.41-1.38 (m, 2H), 1.34$1.28(\mathrm{~m}, 4 \mathrm{H}), 0.90(\mathrm{t}, J=7.0 \mathrm{~Hz}, 3 \mathrm{H}) .{ }^{19} \mathbf{F}$ NMR $\left(565 \mathrm{MHz}, \mathrm{CDCl}_{3}\right) \delta-81.1(\mathrm{t}, J=9.6$ $\mathrm{Hz}, 3 \mathrm{~F}),-96.5$ (m, 2F), -123.6 (m, 2F), -125.5 (m, 2F). 


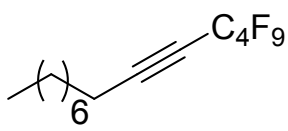

1,1,1,2,2,3,3,4,4-nonafluorotetradec-5-yne (3t). The general procedure for ironcatalyzed perfluoroalkylation reaction as described previously was followed to synthesize $3 \mathbf{t}$ from $\mathbf{1 t}(0.2 \mathrm{mmol}, 28 \mathrm{mg})$ and $\mathbf{2 a}(0.4 \mathrm{mmol}, 70 \mu \mathrm{L}), \mathrm{FeCl}_{2} \bullet 4 \mathrm{H}_{2} \mathrm{O}(0.04$ mmol, $8 \mathrm{mg}), \mathrm{Cs}_{2} \mathrm{CO}_{3}(0.6 \mathrm{mmol}, 196 \mathrm{mg})$ and $2 \mathrm{~mL}$ of $t \mathrm{BuOH}$. The reaction mixture was stirred and heated at $70{ }^{\circ} \mathrm{C}$ (oil bath) for 14 hours, followed by a workup. The residue was purified by flash column chromatography on silica gel (petroleum ether) to afford 3t (16 mg, 22\% yield) as a colorless oil. ${ }^{1} \mathbf{H} \mathbf{~ N M R}\left(600 \mathrm{MHz}, \mathrm{CDCl}_{3}\right) \delta 2.37$ $2.33(\mathrm{~m}, 2 \mathrm{H}), 1.61-1.56(\mathrm{~m}, 2 \mathrm{H}), 1.42-1.37(\mathrm{~m}, 2 \mathrm{H}), 1.31-1.26(\mathrm{~m}, 8 \mathrm{H}), 0.89(\mathrm{t}, J=6.8$ $\mathrm{Hz}, 3 \mathrm{H}) .{ }^{19} \mathbf{F}$ NMR $\left(565 \mathrm{MHz}, \mathrm{CDCl}_{3}\right) \delta(\mathrm{t}, J=9.8 \mathrm{~Hz}, 3 \mathrm{~F}),-96.5(\mathrm{~m}, 2 \mathrm{~F}),-123.6(\mathrm{~m}$, 2F), -125.5 (m, 2F). ${ }^{13} \mathbf{C}\left\{{ }^{1} \mathbf{H}\right\} \mathbf{N M R}\left(151 \mathrm{MHz}, \mathrm{CDCl}_{3}\right) \delta 118.7-109.3\left[\mathrm{~m},-\left(\mathrm{CF}_{2}\right) \mathrm{CF}_{3}\right]$, 108.9-105.3 [m, $\left.-\left(\mathrm{CF}_{2}\right) \mathrm{CF}_{3}\right] 95.6(\mathrm{t}, J=6.2 \mathrm{~Hz}), 67.4(\mathrm{t}, J=35.7 \mathrm{~Hz}), 31.9,29.2,29.0$, 28.8, $27.3(\mathrm{t}, J=2.1 \mathrm{~Hz}), 22.8,18.6(\mathrm{t}, J=2.6 \mathrm{~Hz}), 14.2$. HRMS (EI) m/z: [M] ${ }^{+}$Calcd for $\mathrm{C}_{14} \mathrm{H}_{17} \mathrm{~F}_{9}^{+}:$356.1181; Found 356.1189.

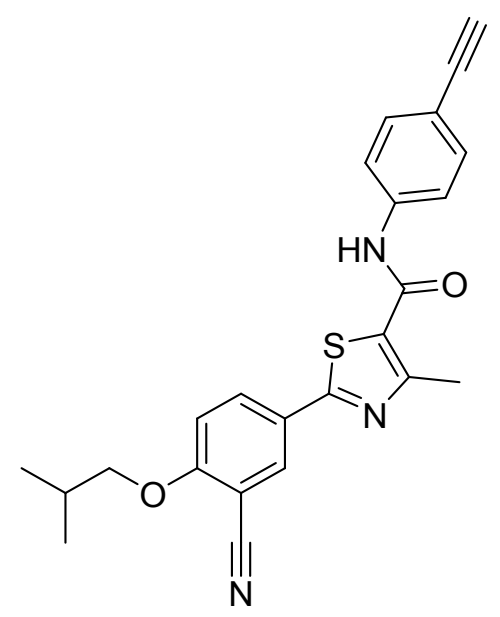

2-(3-cyano-4-isobutoxyphenyl)-N-(4-ethynylphenyl)-4-methylthiazole-5-

carboxamide (1u). The general procedure for the synthesis of substrate $1 \mathbf{u}$ as described previously was followed to synthesize it. The product (170 $\mathrm{mg}, 82 \%$ yield) as a white solid was purified with silica gel chromatography (petroleum ether/ethyl ether $=4: 1$ ). ${ }^{1} \mathbf{H}$ NMR $\left(400 \mathrm{MHz}, \mathrm{CDCl}_{3}\right) \delta 8.07(\mathrm{~d}, J=2.3 \mathrm{~Hz}, 1 \mathrm{H}), 8.00(\mathrm{dd}, J=8.8,2.4 \mathrm{~Hz}, 1 \mathrm{H})$, 7.62-7.47 (m, 3H), $7.43(\mathrm{~d}, J=8.7 \mathrm{~Hz}, 2 \mathrm{H}), 6.95(\mathrm{~d}, J=8.9 \mathrm{~Hz}, 1 \mathrm{H}), 3.83(\mathrm{~d}, J=6.5$ 
$\mathrm{Hz}, 2 \mathrm{H}), 3.01(\mathrm{~s}, 1 \mathrm{H}), 2.71(\mathrm{~s}, 3 \mathrm{H}), 2.13(\mathrm{dt}, J=13.3,6.6 \mathrm{~Hz}, 1 \mathrm{H}), 1.02(\mathrm{~d}, J=6.7 \mathrm{~Hz}$, $6 \mathrm{H}) .{ }^{13} \mathbf{C}\left\{{ }^{1} \mathrm{H}\right\}$ NMR $\left(101 \mathrm{MHz}, \mathrm{CDCl}_{3}\right) \delta 165.1,162.6,159.6,157.5,137.8,133.1$, 132.6, 132.1, 125.7, 125.5, 119.8, 119.5, 118.5, 115.4, 112.7, 103.0, 83.2, 75.7, 28.2, 19.1, 17.6. HRMS (ESI) m/z: $[\mathrm{M}+\mathrm{H}]^{+}$Calcd for $\mathrm{C}_{24} \mathrm{H}_{22} \mathrm{~N}_{3} \mathrm{O}_{2} \mathrm{~S}^{+}$: 416.1427; Found 416.1433 .

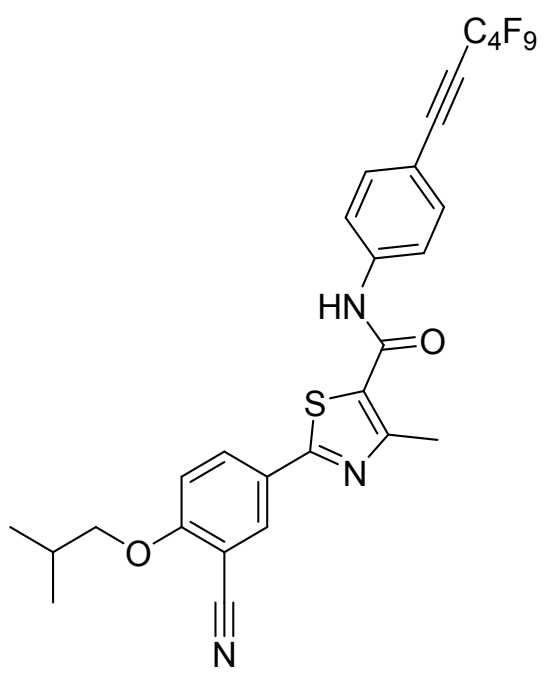

2-(3-cyano-4-isobutoxyphenyl)-4-methyl-N-(4-(perfluorohex-1-yn-1-yl)thiazole-5carboxamide (3u). The general procedure for iron-catalyzed perfluoroalkylation reaction as described previously was followed to synthesize $\mathbf{3} \mathbf{u}$ from $\mathbf{1} \mathbf{u}(0.2 \mathrm{mmol}, 83$ $\mathrm{mg})$ and $2 \mathrm{a}(0.4 \mathrm{mmol}, 70 \mu \mathrm{L}), \mathrm{FeCl}_{2} \bullet 4 \mathrm{H}_{2} \mathrm{O}(0.04 \mathrm{mmol}, 8 \mathrm{mg}), \mathrm{Cs}_{2} \mathrm{CO}_{3}(0.6 \mathrm{mmol}$, $196 \mathrm{mg}$ ) and $2 \mathrm{~mL}$ of $t \mathrm{BuOH}$. The reaction mixture was stirred and heated at $70{ }^{\circ} \mathrm{C}$ (oil bath) for 8 hours, followed by a workup. The residue was purified by flash column chromatography on silica gel (petroleum ether/ethyl ether $=5: 1)$ to afford $\mathbf{3 u}(96 \mathrm{mg}$, $76 \%$ yield) as a pale yellow solid. ${ }^{1} \mathbf{H}$ NMR $\left(600 \mathrm{MHz}, \mathrm{CDCl}_{3}\right) \delta 8.07(\mathrm{~d}, J=2.3 \mathrm{~Hz}$, $1 \mathrm{H}), 8.02(\mathrm{dd}, J=8.8,2.3 \mathrm{~Hz}, 1 \mathrm{H}), 7.63-7.58(\mathrm{~m}, 3 \mathrm{H}), 7.51$ (d, $J=8.6 \mathrm{~Hz}, 2 \mathrm{H}), 6.96$ (d, $J=8.9 \mathrm{~Hz}, 1 \mathrm{H}), 3.84(\mathrm{~d}, J=6.5 \mathrm{~Hz}, 2 \mathrm{H}), 2.73$ (s, 3H), 2.16-2.10 (m, 1H), 1.02 (d, $J=6.7 \mathrm{~Hz}, 6 \mathrm{H}) .{ }^{13} \mathbf{C}\left\{{ }^{1} \mathbf{H}\right\} \mathbf{N M R}\left(151 \mathrm{MHz}, \mathrm{CDCl}_{3}\right) \delta 164.3,161.6,158.7,156.9,138.9$, $132.6(\mathrm{t}, J=2.2 \mathrm{~Hz}), 131.6,131.1,124.5,124.0,118.8,117.3-115.4$ [m, $\left.-\left(\mathrm{CF}_{2}\right) \mathrm{CF}_{3}\right]$, $114.4,113.3(\mathrm{t}, J=2.8 \mathrm{~Hz}), 111.7,108.7-104.8$ [m, $\left.-\left(\mathrm{CF}_{2}\right) \mathrm{CF}_{3}\right], 101.9,90.9$ (t, $J=6.2$ $\mathrm{Hz}), 74.7,73.6(\mathrm{t}, J=36.2 \mathrm{~Hz}), 27.1,18.0,16.6 .{ }^{19} \mathbf{F} \mathbf{N M R}\left(565 \mathrm{MHz}, \mathrm{CDCl}_{3}\right) \delta-80.9$ (t, $J=9.6 \mathrm{~Hz}, 3 \mathrm{~F}),-97.2(\mathrm{~m}, 2 \mathrm{~F}),-123.3(\mathrm{~m}, 2 \mathrm{~F}),-125.4(\mathrm{~m}, 2 \mathrm{~F})$. HRMS (ESI) m/z: 
$[\mathrm{M}+\mathrm{H}]^{+}$Calcd for $\mathrm{C}_{28} \mathrm{H}_{21} \mathrm{~F}_{9} \mathrm{~N}_{3} \mathrm{O}_{2} \mathrm{~S}^{+}$: 634.1205; Found 634.1213.<smiles>FC(F)(F)C#Cc1ccc2ccccc2c1</smiles>

2-(perfluorohex-1-yn-1-yl)naphthalene $\mathbf{( 3 v )}$. The general procedure for ironcatalyzed perfluoroalkylation reaction as described previously was followed to synthesize 3v from $\mathbf{1 v}(0.2 \mathrm{mmol}, 30 \mathrm{mg})$ and $\mathbf{2 a}(0.4 \mathrm{mmol}, 70 \mu \mathrm{L}), \mathrm{FeCl}_{2} \bullet 4 \mathrm{H}_{2} \mathrm{O}(0.04$ mmol, $8 \mathrm{mg}), \mathrm{Cs}_{2} \mathrm{CO}_{3}(0.6 \mathrm{mmol}, 196 \mathrm{mg})$ and $2 \mathrm{~mL}$ of $t \mathrm{BuOH}$. The reaction mixture was stirred and heated at $70{ }^{\circ} \mathrm{C}$ (oil bath) for 8 hours, followed by a workup. The residue was purified by flash column chromatography on silica gel (petroleum ether) to afford 3v (31 mg, 42\% yield) as a colorless oil. This compound is known. ${ }^{1}{ }^{\mathbf{1}} \mathbf{H}$ NMR (600 $\left.\mathrm{MHz} \mathrm{CDCl}_{3}\right) \delta 8.14(\mathrm{~s}, 1 \mathrm{H}), 7.86(\mathrm{~d}, J=8.7 \mathrm{~Hz}, 3 \mathrm{H}), 7.64-7.49(\mathrm{~m}, 3 \mathrm{H}),{ }^{19} \mathbf{F}$ NMR $\left(565 \mathrm{MHz}, \mathrm{CDCl}_{3}\right) \delta-80.9(\mathrm{t}, J=9.4 \mathrm{~Hz}, 3 \mathrm{~F}),-97.2(\mathrm{~m}, 2 \mathrm{~F}),-123.3(\mathrm{~m}, 2 \mathrm{~F}),-125.4(\mathrm{~m}$, 2F).<smiles>COc1ccc(C#CC(F)(F)C(F)(F)F)cc1</smiles>

1-methoxy-4-(perfluoropent-1-yn-1-yl)benzene (5a). The general procedure for ironcatalyzed perfluoroalkylation reaction as described previously was followed to synthesize $\mathbf{5 a}$ from $\mathbf{1 e}(0.2 \mathrm{mmol}, 26 \mathrm{mg})$ and $\mathbf{2 b}(0.4 \mathrm{mmol}, 118 \mathrm{mg}), \mathrm{FeCl}_{2} \bullet 4 \mathrm{H}_{2} \mathrm{O}$ (0.04 mmol, $8 \mathrm{mg}), \mathrm{Cs}_{2} \mathrm{CO}_{3}(0.6 \mathrm{mmol}, 196 \mathrm{mg})$ and $2 \mathrm{~mL}$ of $t \mathrm{BuOH}$. The reaction mixture was stirred and heated at $70{ }^{\circ} \mathrm{C}$ (oil bath) for 8 hours, followed by a workup. The residue was purified by flash column chromatography on silica gel (petroleum ether) to afford $\mathbf{5 a}$ (47 mg, 78\% yield) as a pale yellow oil. This compound is known. ${ }^{5}$ ${ }^{1} \mathbf{H}$ NMR $\left(600 \mathrm{MHz}, \mathrm{CDCl}_{3}\right) \delta 7.42(\mathrm{~d}, J=8.8 \mathrm{~Hz}, 2 \mathrm{H}), 6.82(\mathrm{~d}, J=8.8 \mathrm{~Hz}, 2 \mathrm{H}), 3.76$ (s, 3H). ${ }^{19}$ F NMR (565 MHz, $\left.\mathrm{CDCl}_{3}\right) \delta-80.1(\mathrm{t}, J=8.4 \mathrm{~Hz}, 3 \mathrm{~F}),-97.6(\mathrm{~m}, 2 \mathrm{~F}),-126.9$ (t, $J=6.4 \mathrm{~Hz}, 2 \mathrm{~F})$. 


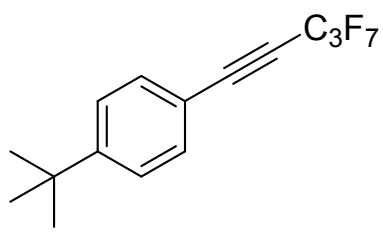

1-(perfluoropent-1-yn-1-yl)-4-tert-butylkylbenzene (5b). The general procedure for iron-catalyzed perfluoroalkylation reaction as described previously was followed to synthesize $5 \mathbf{b}$ from $\mathbf{1 d}(0.2 \mathrm{mmol}, 32 \mathrm{mg})$ and $\mathbf{2 b}(0.4 \mathrm{mmol}, 118 \mathrm{mg}), \mathrm{FeCl}_{2} \bullet 4 \mathrm{H}_{2} \mathrm{O}$ (0.04 mmol, $8 \mathrm{mg}), \mathrm{Cs}_{2} \mathrm{CO}_{3}(0.6 \mathrm{mmol}, 196 \mathrm{mg})$ and $2 \mathrm{~mL}$ of $t \mathrm{BuOH}$. The reaction mixture was stirred and heated at $70{ }^{\circ} \mathrm{C}$ (oil bath) for 8 hours, followed by a workup. The residue was purified by flash column chromatography on silica gel (petroleum ether) to afford $\mathbf{5 b}$ (47 mg, $72 \%$ yield) as a pale yellow oil. This compound is known. ${ }^{5}$ ${ }^{1} \mathbf{H}$ NMR $\left(600 \mathrm{MHz}, \mathrm{CDCl}_{3}\right) \delta 7.42(\mathrm{~d}, J=8.4 \mathrm{~Hz}, 2 \mathrm{H}), 7.34(\mathrm{~d}, J=8.4 \mathrm{~Hz}, 2 \mathrm{H}), 1.25$ (s, 9H). ${ }^{19}$ F NMR (565 MHz, $\left.\mathrm{CDCl}_{3}\right) \delta-80.1$ (t, $\left.J=8.4 \mathrm{~Hz}, 3 \mathrm{~F}\right),-97.9(\mathrm{~m}, 2 \mathrm{~F}),-126.9$ $(\mathrm{t}, J=6.1 \mathrm{~Hz}, 2 \mathrm{~F})$.

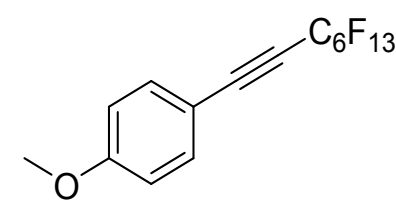

1-methoxy-4-(perfluorooct-1-yn-1-yl)benzene (6a). The general procedure for ironcatalyzed perfluoroalkylation reaction as described previously was followed to synthesize 6a from 1 e $(0.2 \mathrm{mmol}, 26 \mathrm{mg})$ and $\mathbf{2 c}(0.4 \mathrm{mmol}, 178 \mathrm{mg}), \mathrm{FeCl}_{2} \bullet 4 \mathrm{H}_{2} \mathrm{O}$ (0.04 mmol, $8 \mathrm{mg}), \mathrm{Cs}_{2} \mathrm{CO}_{3}(0.6 \mathrm{mmol}, 196 \mathrm{mg})$ and $2 \mathrm{~mL}$ of $t \mathrm{BuOH}$. The reaction mixture was stirred and heated at $70{ }^{\circ} \mathrm{C}$ (oil bath) for 8 hours, followed by a workup. The residue was purified by flash column chromatography on silica gel (petroleum ether) to afford $\mathbf{6 a}\left(70 \mathrm{mg}, 78 \%\right.$ yield) as a pale yellow oil. This compound is known. ${ }^{1}$ ${ }^{1} \mathbf{H}$ NMR $\left(600 \mathrm{MHz}, \mathrm{CDCl}_{3}\right) \delta 7.42(\mathrm{~d}, J=8.8 \mathrm{~Hz}, 2 \mathrm{H}), 6.82(\mathrm{~d}, J=8.8 \mathrm{~Hz}, 2 \mathrm{H}), 3.76$ (s, 3H). ${ }^{19}$ F NMR $\left(565 \mathrm{MHz}, \mathrm{CDCl}_{3}\right) \delta-80.9$ (q, $\left.J=9.0 \mathrm{~Hz}, 3 \mathrm{~F}\right),-96.5(\mathrm{~s}, 2 \mathrm{~F}),-121.4$ (m, 2F), -122.5 (d, $J=12.8 \mathrm{~Hz}, 2 \mathrm{~F}),-122.9(\mathrm{t}, J=14.7 \mathrm{~Hz}, 2 \mathrm{~F}),-126.2(\mathrm{t}, J=15.2 \mathrm{~Hz}$, 2F). 
<smiles>CCCCc1ccc(C#CC(F)(F)F)cc1</smiles>

1-(perfluorooct-1-yn-1-yl)-4-butylalkylbenzene (6b). The general procedure for iron-catalyzed perfluoroalkylation reaction as described previously was followed to synthesize $\mathbf{6 b}$ from $\mathbf{1 c}(0.2 \mathrm{mmol}, 32 \mathrm{mg})$ and $\mathbf{2 c}(0.4 \mathrm{mmol}, 178 \mathrm{mg}), \mathrm{FeCl}_{2} \cdot 4 \mathrm{H}_{2} \mathrm{O}$ (0.04 mmol, $8 \mathrm{mg}), \mathrm{Cs}_{2} \mathrm{CO}_{3}(0.6 \mathrm{mmol}, 196 \mathrm{mg})$ and $2 \mathrm{~mL}$ of $t \mathrm{BuOH}$. The reaction mixture was stirred and heated at $70{ }^{\circ} \mathrm{C}$ (oil bath) for 8 hours, followed by a workup. The residue was purified by flash column chromatography on silica gel (petroleum ether) to afford $\mathbf{6 b}\left(55 \mathrm{mg}, 58 \%\right.$ yield) as a pale yellow oil. ${ }^{1} \mathbf{H} \mathbf{N M R}\left(600 \mathrm{MHz}, \mathrm{CDCl}_{3}\right)$ $\delta 7.39(\mathrm{~d}, J=7.9 \mathrm{~Hz}, 2 \mathrm{H}), 7.13(\mathrm{~d}, J=7.9 \mathrm{~Hz}, 2 \mathrm{H}), 2.56(\mathrm{t}, J=7.8 \mathrm{~Hz}, 2 \mathrm{H}), 1.61-1.45$ $(\mathrm{m}, 2 \mathrm{H}), 1.27(\mathrm{~h}, J=7.4 \mathrm{~Hz}, 2 \mathrm{H}), 0.85$ (t, $J=7.4 \mathrm{~Hz}, 3 \mathrm{H}) .{ }^{13} \mathbf{C}\left\{{ }^{1} \mathbf{H}\right\}$ NMR $(151 \mathrm{MHz}$, $\left.\mathrm{CDCl}_{3}\right) \delta 146.7,132.4(\mathrm{t}, J=2.4 \mathrm{~Hz}), 128.8,118.2-116.3\left[\mathrm{~m},-\left(\mathrm{CF}_{2}\right) \mathrm{CF}_{3}\right], 115.6(\mathrm{t}, J=$ $3.0 \mathrm{~Hz}), 114.6-105.8$ [m, $\left.-\left(\mathrm{CF}_{2}\right) \mathrm{CF}_{3}\right], 92.8(\mathrm{t}, J=6.3 \mathrm{~Hz}), 74.2(\mathrm{t}, J=36.0 \mathrm{~Hz}), 35.8$, 33.2, 22.3, 13.9. ${ }^{19} \mathbf{F}$ NMR $\left(565 \mathrm{MHz}, \mathrm{CDCl}_{3}\right) \delta-80.8(\mathrm{t}, J=10.0 \mathrm{~Hz}, 3 \mathrm{~F}),-96.8(\mathrm{~m}$, 2F), -121.3 (m, 2F), -122.5 (m, 2F), -122.8 (m, 2F), -126.2 (m, 2F). HRMS (EI) m/z: $[\mathrm{M}]^{+}$Calcd for $\mathrm{C}_{18} \mathrm{H}_{13} \mathrm{~F}_{13}{ }^{+}$: 476.0804; Found 476.0812.<smiles>Fc1ccc(C#CC(F)(F)F)cc1</smiles>

1-fluoro-4-(perfluorooct -1-yn-1-yl)benzene (6c). The general procedure for ironcatalyzed perfluoroalkylation reaction as described previously was followed to synthesize 6c from $1 \mathbf{f}(0.2 \mathrm{mmol}, 24 \mathrm{mg})$ and $\mathbf{2 c}(0.4 \mathrm{mmol}, 178 \mathrm{mg}), \mathrm{FeCl}_{2} \bullet 4 \mathrm{H}_{2} \mathrm{O}(0.04$ mmol, $8 \mathrm{mg}), \mathrm{Cs}_{2} \mathrm{CO}_{3}(0.6 \mathrm{mmol}, 196 \mathrm{mg})$ and $2 \mathrm{~mL}$ of $t \mathrm{BuOH}$. The reaction mixture was stirred and heated at $70{ }^{\circ} \mathrm{C}$ (oil bath) for 14 hours, followed by a workup. The residue was purified by flash column chromatography on silica gel (petroleum ether) to afford $6 \mathbf{c}$ (53 mg, 61\% yield) as a pale yellow oil. This compound is known. ${ }^{1} \mathbf{H}$ NMR $\left(600 \mathrm{MHz}, \mathrm{CDCl}_{3}\right) \delta 7.50(\mathrm{dd}, J=8.7,5.3 \mathrm{~Hz}, 2 \mathrm{H}), 7.03(\mathrm{t}, J=8.6 \mathrm{~Hz}, 2 \mathrm{H}) .{ }^{19} \mathbf{F}$ NMR $\left(565 \mathrm{MHz} \mathrm{CDCl}_{3}\right) \delta-80.8(\mathrm{t}, J=10.0 \mathrm{~Hz}, 3 \mathrm{~F}),-97.3(\mathrm{t}, J=9.1 \mathrm{~Hz}, 2 \mathrm{~F}),-105.6(\mathrm{~s}, 1 \mathrm{~F})$, 
$-121.3(\mathrm{~m}, 2 \mathrm{~F}),-122.5(\mathrm{~m}, 2 \mathrm{~F}),-122.5(\mathrm{~m}, 2 \mathrm{~F}),-126.1(\mathrm{~m}, 2 \mathrm{~F})$.<smiles>COc1ccc(C#CC(F)F)cc1</smiles>

1-methoxy-4-(perfluorooctyl-1-yn-1-yl)benzene (7a). The general procedure for iron-catalyzed perfluoroalkylation reaction as described previously was followed to synthesize $7 \mathbf{a}$ from 1 e $(0.2 \mathrm{mmol}, 26 \mathrm{mg})$ and $\mathbf{2 d}(0.4 \mathrm{mmol}, 218 \mathrm{mg}), \mathrm{FeCl}_{2} \bullet 4 \mathrm{H}_{2} \mathrm{O}$ (0.04 mmol, $8 \mathrm{mg}), \mathrm{Cs}_{2} \mathrm{CO}_{3}(0.6 \mathrm{mmol}, 196 \mathrm{mg})$ and $2 \mathrm{~mL}$ of $t \mathrm{BuOH}$. The reaction mixture was stirred and heated at $70{ }^{\circ} \mathrm{C}$ (oil bath) for 8 hours, followed by a workup. The residue was purified by flash column chromatography on silica gel (petroleum ether) to afford $7 \mathbf{a}\left(91 \mathrm{mg}, 83 \%\right.$ yield) as a pale yellow oil. ${ }^{1} \mathbf{H} \mathbf{~ N M R}\left(600 \mathrm{MHz}, \mathrm{CDCl}_{3}\right)$ $\delta 7.43(\mathrm{~d}, J=8.8 \mathrm{~Hz}, 2 \mathrm{H}), 6.83(\mathrm{~d}, J=8.8 \mathrm{~Hz}, 2 \mathrm{H}), 3.77(\mathrm{~s}, 3 \mathrm{H}) .{ }^{13} \mathbf{C}\left\{{ }^{1} \mathbf{H}\right\}$ NMR $(151$ $\left.\mathrm{MHz}, \mathrm{CDCl}_{3}\right) \delta 161.7,134.3(\mathrm{t}, J=2.4 \mathrm{~Hz}), 118.1-116.2$ [m, $\left.-\left(\mathrm{CF}_{2}\right) \mathrm{CF}_{3}\right], 114.3,112.8-$ $105.9\left[\mathrm{~m},-\left(\mathrm{CF}_{2}\right) \mathrm{CF}_{3}\right], 110.3(\mathrm{t}, J=3.1 \mathrm{~Hz}), 92.8(\mathrm{t}, J=6.3 \mathrm{~Hz}), 73.8(\mathrm{t}, J=36.0 \mathrm{~Hz})$, 55.4. ${ }^{19}$ F NMR $\left(565 \mathrm{MHz}, \mathrm{CDCl}_{3}\right) \delta-80.8(\mathrm{t}, J=10.1 \mathrm{~Hz}, 3 \mathrm{~F}),-96.5(\mathrm{~m}, 2 \mathrm{~F}),-121.1$ (m, 2F), -121.9 (m, 4F), -122.4 (m, 2F), -122.8 (m, 2F), -126.2 (m, 2F). HRMS (ESI) $\mathbf{m} / \mathbf{z}:[\mathrm{M}+\mathrm{H}]^{+}$Calcd for $\mathrm{C}_{17} \mathrm{H}_{8} \mathrm{~F}_{17} \mathrm{O}^{+}$: 551.0298; Found 551.0300.

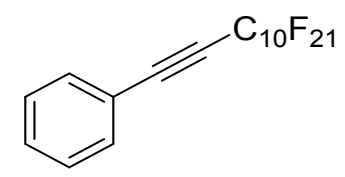

(perfluorodecyl-1-yn-1-yl)benzene (8a). The general procedure for iron-catalyzed perfluoroalkylation reaction as described previously was followed to synthesize 8a from 1a $(0.2 \mathrm{mmol}, 20 \mathrm{mg})$ and $\mathbf{2 e}(0.4 \mathrm{mmol}, 258 \mathrm{mg}), \mathrm{FeCl}_{2} \bullet 4 \mathrm{H}_{2} \mathrm{O}(0.04 \mathrm{mmol}, 8$ $\mathrm{mg}), \mathrm{Cs}_{2} \mathrm{CO}_{3}(0.6 \mathrm{mmol}, 196 \mathrm{mg})$ and $2 \mathrm{~mL}$ of $t \mathrm{BuOH}$. The reaction mixture was stirred and heated at $70{ }^{\circ} \mathrm{C}$ (oil bath) for 8 hours, followed by a workup. The residue was purified by flash column chromatography on silica gel (petroleum ether) to afford 8a (105 mg, 85\% yield) as a pale yellow oil. This compound is known. ${ }^{6} \mathbf{H}$ NMR (600 $\left.\mathrm{MHz}, \mathrm{CDCl}_{3}\right) \delta 7.49(\mathrm{~d}, J=6.7 \mathrm{~Hz}, 2 \mathrm{H}), 7.42(\mathrm{t}, J=7.5 \mathrm{~Hz}, 1 \mathrm{H}), 7.33(\mathrm{t}, J=7.7 \mathrm{~Hz}$, 
2H). ${ }^{19}$ F NMR $\left(565 \mathrm{MHz}, \mathrm{CDCl}_{3}\right) \delta-80.8(\mathrm{t}, J=9.9 \mathrm{~Hz}, 3 \mathrm{~F}),-97.1(\mathrm{t}, J=8.7 \mathrm{~Hz}, 2 \mathrm{~F})$, $-121.0(\mathrm{~m}, 2 \mathrm{~F}),-121.7$ (m, 6F), -122.0 (m, 2F), -122.5 (m, 2F), -122.7(m, 2F), -126.2 $(\mathrm{m}, 2 \mathrm{~F})$.<smiles>COc1ccc(C#CC(F)F)cc1</smiles>

1-methoxy-4-(perfluorodecyl-1-yn-1-yl)benzene $(\mathbf{8 b})$. The general procedure for iron-catalyzed perfluoroalkylation reaction as described previously was followed to synthesize $\mathbf{8 b}$ from $\mathbf{1 e}(0.2 \mathrm{mmol}, 26 \mathrm{mg})$ and $\mathbf{2 e}(0.4 \mathrm{mmol}, 258 \mathrm{mg}), \mathrm{FeCl}_{2} \bullet 4 \mathrm{H}_{2} \mathrm{O}$ (0.04 mmol, $8 \mathrm{mg}), \mathrm{Cs}_{2} \mathrm{CO}_{3}(0.6 \mathrm{mmol}, 196 \mathrm{mg})$ and $2 \mathrm{~mL}$ of $t \mathrm{BuOH}$. The reaction mixture was stirred and heated at $70{ }^{\circ} \mathrm{C}$ (oil bath) for 8 hours, followed by a workup. The residue was purified by flash column chromatography on silica gel (petroleum ether) to afford $\mathbf{8 b}$ (106 mg, 82\% yield) as a pale yellow oil. This compound is known. ${ }^{7}$ ${ }^{1} \mathbf{H}$ NMR $\left(600 \mathrm{MHz}, \mathrm{CDCl}_{3}\right) \delta 7.43(\mathrm{~d}, J=8.8 \mathrm{~Hz}, 2 \mathrm{H}), 6.83(\mathrm{~d}, J=8.8 \mathrm{~Hz}, 2 \mathrm{H}), 3.77$ $(\mathrm{s}, 3 \mathrm{H}) .{ }^{19} \mathbf{F}$ NMR $\left(565 \mathrm{MHz}, \mathrm{CDCl}_{3}\right) \delta-80.8(\mathrm{t}, J=9.9 \mathrm{~Hz}, 3 \mathrm{~F}),-96.5(\mathrm{~m}, 2 \mathrm{~F}),-121.1$ (m, 2F), -121.7 (m, 6F), -121.9 (m, 2F), -122.5 (m, 2F), -122.8 (m, 2F), -126.1 (m, 2F).

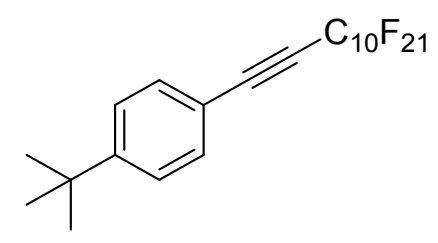

1-( perfluorodecyl -1-yn-1-yl)-4-tert-butylkylbenzene (8c). The general procedure for iron-catalyzed perfluoroalkylation reaction as described previously was followed to synthesize $8 \mathbf{c}$ from $\mathbf{1 d}(0.2 \mathrm{mmol}, 32 \mathrm{mg})$ and $\mathbf{2 e}(0.4 \mathrm{mmol}, 258 \mathrm{mg}), \mathrm{FeCl}_{2} \bullet 4 \mathrm{H}_{2} \mathrm{O}$ (0.04 mmol, $8 \mathrm{mg}), \mathrm{Cs}_{2} \mathrm{CO}_{3}(0.6 \mathrm{mmol}, 196 \mathrm{mg})$ and $2 \mathrm{~mL}$ of $t \mathrm{BuOH}$. The reaction mixture was stirred and heated at $70{ }^{\circ} \mathrm{C}$ (oil bath) for 8 hours, followed by a workup. The residue was purified by flash column chromatography on silica gel (petroleum ether) to afford $\mathbf{8 c}\left(106 \mathrm{mg}, 78 \%\right.$ yield) as a white solid. ${ }^{1} \mathbf{H}$ NMR (600 $\left.\mathrm{MHz}, \mathrm{CDCl}_{3}\right)$ $\delta 7.42(\mathrm{~d}, J=8.5 \mathrm{~Hz}, 2 \mathrm{H}), 7.34(\mathrm{~d}, J=8.5 \mathrm{~Hz}, 2 \mathrm{H}), 1.24(\mathrm{~s}, 9 \mathrm{H}),{ }^{13} \mathbf{C}\left\{{ }^{1} \mathbf{H}\right\} \mathbf{N M R}(151$ $\left.\mathrm{MHz}, \mathrm{CDCl}_{3}\right) \delta 154.8,132.3(\mathrm{t}, J=2.5 \mathrm{~Hz}), 125.7,118.3-115.9\left[\mathrm{~m},-\left(\mathrm{CF}_{2}\right) \mathrm{CF}_{3}\right], 115.4$ 
(t, $J=2.6 \mathrm{~Hz}), 114.5-105.8\left[\mathrm{~m},-\left(\mathrm{CF}_{2}\right) \mathrm{CF}_{3}\right], 92.7(\mathrm{t}, J=6.3 \mathrm{~Hz}), 74.2(\mathrm{t}, J=36.1 \mathrm{~Hz})$, 35.1, 31.0. ${ }^{19}$ F NMR $\left(565 \mathrm{MHz}, \mathrm{CDCl}_{3}\right) \delta-80.9(\mathrm{t}, J=9.7 \mathrm{~Hz}, 3 \mathrm{~F}),-96.9(\mathrm{~m}, 2 \mathrm{~F}),-$ $121.2(\mathrm{~m}, 2 \mathrm{~F}),-121.8(\mathrm{~m}, 6 \mathrm{~F}),-122.0$ (m, 2F), -122.5 (m, 2F), -122.8 (m, 2F), -126.2 (m, 2F). HRMS (EI) m/z: [M] $]^{+}$Calcd for $\mathrm{C}_{22} \mathrm{H}_{13} \mathrm{~F}_{21}{ }^{+}$: 676.0676; Found 676.0683.

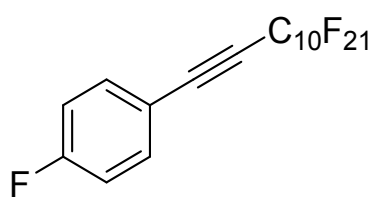

1-fluoro-4-(perfluorodecyl-1-yn-1-yl)benzene (8d). The general procedure for ironcatalyzed perfluoroalkylation reaction as described previously was followed to synthesize 8d from 1 f $(0.2 \mathrm{mmol}, 24 \mathrm{mg})$ and $\mathbf{2 e}(0.4 \mathrm{mmol}, 258 \mathrm{mg}), \mathrm{FeCl}_{2} \bullet 4 \mathrm{H}_{2} \mathrm{O}$ (0.04 mmol, $8 \mathrm{mg}), \mathrm{Cs}_{2} \mathrm{CO}_{3}(0.6 \mathrm{mmol}, 196 \mathrm{mg})$ and $2 \mathrm{~mL}$ of $t \mathrm{BuOH}$. The reaction mixture was stirred and heated at $70{ }^{\circ} \mathrm{C}$ (oil bath) for 14 hours, followed by a workup. The residue was purified by flash column chromatography on silica gel (petroleum ether) to afford $\mathbf{8 d}$ (79 $\mathrm{mg}, 62 \%$ yield) as a white solid. This compound is known. ${ }^{8}{ }^{1} \mathbf{H}$ NMR $\left(600 \mathrm{MHz}, \mathrm{CDCl}_{3}\right) \delta 7.48(\mathrm{dd}, J=8.7,5.3 \mathrm{~Hz}, 2 \mathrm{H}), 7.02(\mathrm{t}, J=8.6 \mathrm{~Hz}, 2 \mathrm{H}) .{ }^{19} \mathbf{F}$ NMR $\left(565 \mathrm{MHz}, \mathrm{CDCl}_{3}\right) \delta-81.0(\mathrm{t}, J=10.0 \mathrm{~Hz}, 3 \mathrm{~F}),-97.4(\mathrm{~m}, 2 \mathrm{~F}),-105.8(\mathrm{~m}, 1 \mathrm{~F}),-$ $121.2(\mathrm{~m}, 2 \mathrm{~F}),-121.9(\mathrm{~m}, 6 \mathrm{~F}),-122.1$ (m, 2F), -122.6 (m, 2F), -122.9 (m, 2F), -126.3 $(\mathrm{m}, 2 \mathrm{~F})$.

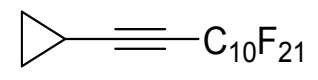

(perfluorodecyl-1-yn-1-yl)cyclopropyl acetylene (8e). The general procedure for iron-catalyzed perfluoroalkylation reaction as described previously was followed to synthesize 8e from $\mathbf{1 w}(0.2 \mathrm{mmol}, 13 \mathrm{mg})$ and $\mathbf{2 e}(0.4 \mathrm{mmol}, 258 \mathrm{mg}), \mathrm{FeCl}_{2} \bullet 4 \mathrm{H}_{2} \mathrm{O}$ (0.04 mmol, $8 \mathrm{mg}), \mathrm{Cs}_{2} \mathrm{CO}_{3}(0.6 \mathrm{mmol}, 196 \mathrm{mg})$ and $2 \mathrm{~mL}$ of $t \mathrm{BuOH}$. The reaction mixture was stirred and heated at $70{ }^{\circ} \mathrm{C}$ (oil bath) for 14 hours, followed by a workup. The residue was purified by flash column chromatography on silica gel (petroleum ether) to afford $\mathbf{8 e}(23 \mathrm{mg}, 20 \%$ yield $)$ as a pale yellow oil. ${ }^{1} \mathbf{H}$ NMR $\left(600 \mathrm{MHz}, \mathrm{CDCl}_{3}\right)$ $\delta$ 1.42-1.36 (m, 1H), 0.98-0.94 (m, 2H), 0.91-0.88 (m, 2H). ${ }^{13} \mathbf{C}\left\{{ }^{1} \mathbf{H}\right\}$ NMR (151 MHz, $\left.\mathrm{CDCl}_{3}\right) \delta 118.3-116.9\left[\mathrm{~m},-\left(\mathrm{CF}_{2}\right) \mathrm{CF}_{3}\right], 111.1-106.8\left[\mathrm{~m},-\left(\mathrm{CF}_{2}\right) \mathrm{CF}_{3}\right], 98.3(\mathrm{t}, J=6.3$ 
$\mathrm{Hz}), 62.1(\mathrm{t}, J=36.1 \mathrm{~Hz}), 9.0,-0.8(\mathrm{t}, J=2.7 \mathrm{~Hz}) .{ }^{19} \mathbf{F}$ NMR $\left(565 \mathrm{MHz}, \mathrm{CDCl}_{3}\right) \delta-80.8$ $(\mathrm{t}, J=10.0 \mathrm{~Hz}, 3 \mathrm{~F}),-95.7(\mathrm{~m}, 2 \mathrm{~F}),-121.2(\mathrm{~m}, 2 \mathrm{~F}),-121.7(\mathrm{~m}, 6 \mathrm{~F}),-121.9(\mathrm{~m}, 2 \mathrm{~F}),-$ $122.6(\mathrm{~m}, 2 \mathrm{~F}),-122.7(\mathrm{~m}, 2 \mathrm{~F}),-126.3(\mathrm{~m}, 2 \mathrm{~F})$. HRMS (EI) m/z: [M] ${ }^{+}$Calcd for $\mathrm{C}_{15} \mathrm{H}_{5} \mathrm{~F}_{21}^{+}$: 584.0050; Found 584.0056.

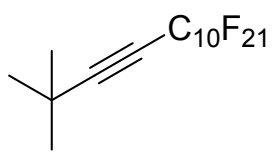

(perfluorodecyl-1-yn-1-yl)-3,3-dimethyl-1-butyne (8f). The general procedure for iron-catalyzed perfluoroalkylation reaction as described previously was followed to synthesize $\mathbf{8 f}$ from $\mathbf{1 x}(0.2 \mathrm{mmol}, 16 \mathrm{mg})$ and $\mathbf{2 e}(0.4 \mathrm{mmol}, 258 \mathrm{mg}), \mathrm{FeCl}_{2} \bullet 4 \mathrm{H}_{2} \mathrm{O}(0.04$ mmol, $8 \mathrm{mg}), \mathrm{Cs}_{2} \mathrm{CO}_{3}(0.6 \mathrm{mmol}, 196 \mathrm{mg})$ and $2 \mathrm{~mL}$ of $t \mathrm{BuOH}$. The reaction mixture was stirred and heated at $70{ }^{\circ} \mathrm{C}$ (oil bath) for 14 hours, followed by a workup. The residue was purified by flash column chromatography on silica gel (petroleum ether) to afford $\mathbf{8 f}$ (17 mg, 14\% yield) as a pale yellow oil. ${ }^{1} \mathbf{H}$ NMR $\left(600 \mathrm{MHz}, \mathrm{CDCl}_{3}\right) \delta 1.28$ (s, 9H). ${ }^{13} \mathbf{C}\left\{{ }^{1} \mathbf{H}\right\}$ NMR $\left(151 \mathrm{MHz}, \mathrm{CDCl}_{3}\right) \delta 118.2-116.1\left[\mathrm{~m},-\left(\mathrm{CF}_{2}\right) \mathrm{CF}_{3}\right], 111.1-106.8$ [m, - $\left.\left(\mathrm{CF}_{2}\right) \mathrm{CF}_{3}\right], 102.7$. (t, $\left.J=6.4 \mathrm{~Hz}\right), 66.2(\mathrm{t}, J=35.9 \mathrm{~Hz}), 29.7(\mathrm{t}, J=2.2 \mathrm{~Hz}), 27.7(\mathrm{t}$, $J=2.1 \mathrm{~Hz}) .{ }^{19} \mathbf{F}$ NMR $\left(565 \mathrm{MHz}, \mathrm{CDCl}_{3}\right) \delta-80.9(\mathrm{t}, J=10.0 \mathrm{~Hz}, 3 \mathrm{~F}),-96.6(\mathrm{~m}, 2 \mathrm{~F}),-$ $121.1(\mathrm{~m}, 2 \mathrm{~F}),-121.8(\mathrm{~m}, 6 \mathrm{~F}),-122.0(\mathrm{~m}, 2 \mathrm{~F}),-122.8$ (m, 2F), -122.9 (m, 2F), -126.2 (m, 2F). HRMS (EI) m/z: [M] ${ }^{+}$Calcd for $\mathrm{C}_{16} \mathrm{H}_{9} \mathrm{~F}_{21}{ }^{+}$: 600.0363; Found 600.0369.

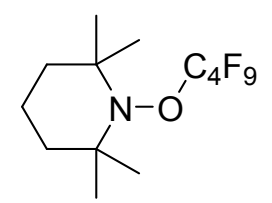

2,2,6,6-tetramethyl-1-(perfluorobutoxy)piperidine (9). The general procedure for iron-catalyzed perfluoroalkylation reaction as described previously was followed to synthesize 9 from $1 \mathbf{a}(0.2 \mathrm{mmol}, 22 \mu \mathrm{L})$ and $\mathbf{2 a}(0.4 \mathrm{mmol}, 70 \mu \mathrm{L}), \mathrm{FeCl}_{2} \bullet 4 \mathrm{H}_{2} \mathrm{O}(0.04$ mmol, $8 \mathrm{mg}), \mathrm{Cs}_{2} \mathrm{CO}_{3}(0.6 \mathrm{mmol}, 196 \mathrm{mg})$, TEMPO (0.2 mmol, $\left.31 \mathrm{mg}\right)$ and $2 \mathrm{~mL}$ of $t \mathrm{BuOH}$. The reaction mixture was stirred and heated at $70{ }^{\circ} \mathrm{C}$ (oil bath) for 8 hours, Then, the reaction mixture was cooled to room temperature. The resulting mixture was diluted and detected by ${ }^{19} \mathrm{~F}$ NMR and HRMS. This compound is known. ${ }^{1}$ This 
compound is known. ${ }^{9}{ }^{19} \mathbf{F}$ NMR $\left(565 \mathrm{MHz}, \mathrm{CDCl}_{3}\right) \delta-77.2(\mathrm{~m}, 2 \mathrm{~F}),-79.8(\mathrm{~m}, 3 \mathrm{~F}),-$ $123.1(\mathrm{~m}, 2 \mathrm{~F}),-124.6(\mathrm{~m}, 2 \mathrm{~F})$ ). HRMS (ESI) m/z: $[\mathrm{M}+\mathrm{H}]^{+}$Calcd for $\mathrm{C}_{13} \mathrm{H}_{19} \mathrm{~F}_{9} \mathrm{NO}^{+}$: 376.1317; Found 376.1278.<smiles>FC(F)(F)CC1=CCCc2ccccc21</smiles>

4-((perfluorohex-1-yn-1-yl))-1,2-dihydronaphthalene (11). The procedure for radical clock experiment as described previously was followed to synthesize 11. The This compound is known. ${ }^{10}$ The product ( $27 \mathrm{mg}, 37 \%$ yield) as a pale yellow oil was purified with silica gel chromatography (petroleum ether). ${ }^{1} \mathbf{H} \mathbf{~ N M R}\left(400 \mathrm{MHz}, \mathrm{CDCl}_{3}\right)$ d 7.28-7.27 (m, 3H), 7.24-7.21 (m, 1H), 5.90 (t, $J=7.3 \mathrm{~Hz}, 1 \mathrm{H}), 3.30$ (t, $J=18.8 \mathrm{~Hz}$, $2 \mathrm{H}), 3.26(\mathrm{t}, J=7.0 \mathrm{~Hz}, 2 \mathrm{H}), 2.76(\mathrm{q}, J=7.0 \mathrm{~Hz}, 2 \mathrm{H}) .{ }^{19} \mathbf{F} \mathbf{N M R}\left(376 \mathrm{MHz}, \mathrm{CDCl}_{3}\right) \delta$ $-81.03(\mathrm{~m}),-111.56(\mathrm{~m}),-124.11(\mathrm{~m}),-125.88(\mathrm{~m})$. 


\section{References}

(1) Li, J.; Liu, L.; Zheng, K.; Zheng, C.; Xiao, H.; Fan, S. Silver-Mediated Perfluoroalkylation of Terminal Alkynes with Perfluoroalkyl Iodides. J. Org. Chem. 2020, 85, 8723-8731.

(2) Yerien, D. E.; Cooke, M. V.; García Vior, M. C.; Barata-Vallejo, S.; Postigo, A. Radical Fluoroalkylation Reactions of (Hetero)Arenes and Sulfides under Red Light Photocatalysis. Org. Biomol. Chem. 2019, 17, 3741-3746.

(3) Xiao, Y.; Chun, Y.-K.; Cheng, S.-C.; Liu, R.; Tse, M.-K.; Ko, C.-C. Visible Light Photocatalytic Cross-Coupling and Adition Reactions of Arylalkynes with Perfluoroalkyl Iodides. Org. Biomol. Chem. 2020, $18,8686-8693$.

(4) Konno, T.; Chae, J.; Kanda, M.; Nagai, G.; Tamura, K.; Ishihara, T.; Yamanaka, H. Facile syntheses of various per- or polyfluoroalkylated internal acetylene derivatives. Tetrahedron. 2003, 59, 7571-7580.

(5) Huang, Y.; Ajitha, M. J.; Huang, K.-W.; Zhang, Z.; Weng, Z. A Class of Effective Decarboxylative Perfluoroalkylating Reagents: $\left[(\mathrm{Phen})_{2} \mathrm{Cu}\right]\left(\mathrm{O}_{2} \mathrm{CR}_{\mathrm{F}}\right)$. Dalton Trans. 2016, 45, 8468-8474.

(6) Wu, W.; Cui, E.; Zhang, Y.; Zhang, C.; Zhu, F.; Tung, C.-H.; Wang, Y. Involving Single-Atom Silver(0) in Selective Dehalogenation by AgF under Visible-Light Irradiation. ACS Catal. 2019, 9, 6335 6341.

(7) Zhang, S.; Rotta-Loria, N.; Weniger, F.; Rabeah, J.; Neumann, H.; Taeschler, C.; Beller, M. A General and Practical Ni-Catalyzed C-H Perfluoroalkylation of (Hetero)Arenes. Chem. Commun. 2019, $55,6723-6726$.

(8) Cowell, A. B.; Tamborski, C. Fluoroalkylation of Aromatic Compounds. J. Fluor. Chem. 1981, 17, $345-356$.

(9) Guo, Q. P.; Wang, M.. R.; Peng, Q.; Huo, Y. M.; Liu, Q.; Wang, R.; Xu, Z. Q. The Dual-functional Chiral Cu-catalyst Induced Photoredox Asymmetric Cyanofluoroalkylation of Alkenes. ACS Catal. 2019, $9,4470-4476$.

(10) He, W.; Yu, J.; Wang, D.; Ran, G.; Xia, X.-F. Synthesis of Tri-Substituted Allyl Alcohols via a Copper/Iron co-Catalyzed Cascade Perfluoroalkylation/Rearrangement of Aryl Propynyl Ethers. Org. Chem. Front. 2019, 6, 3575-3579. 


\section{NMR spectra}

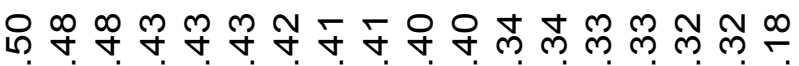

$\wedge N \wedge N \wedge N \wedge N$ N N N N

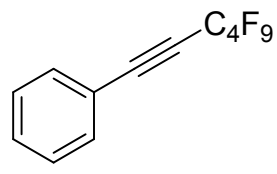

$3 a$

${ }^{1} \mathrm{H} \mathrm{NMR}\left(\mathrm{CDCl}_{3}, 600 \mathrm{MHz}\right)$

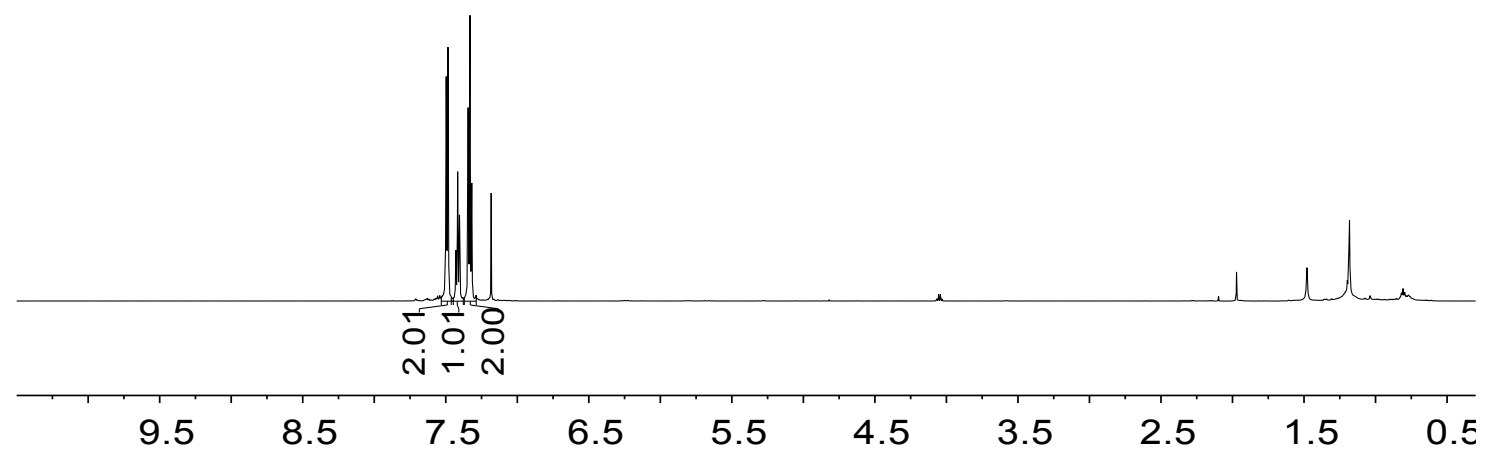

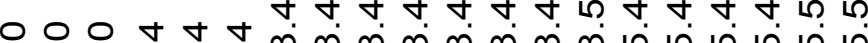
i

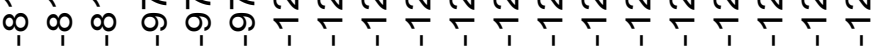

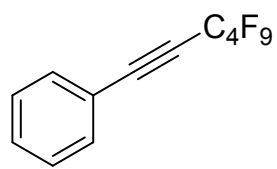

$3 a$

${ }^{19} \mathrm{~F} \mathrm{NMR}\left(\mathrm{CDCl}_{3}, 565 \mathrm{MHz}\right)$

\begin{tabular}{lllllllll}
\hline-30 & -50 & -70 & -90 & -110 & -130 & -150 & -170 & -1
\end{tabular}




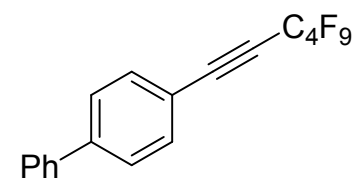

3b

${ }^{1} \mathrm{H} \mathrm{NMR}\left(\mathrm{CDCl}_{3}, 600 \mathrm{MHz}\right)$

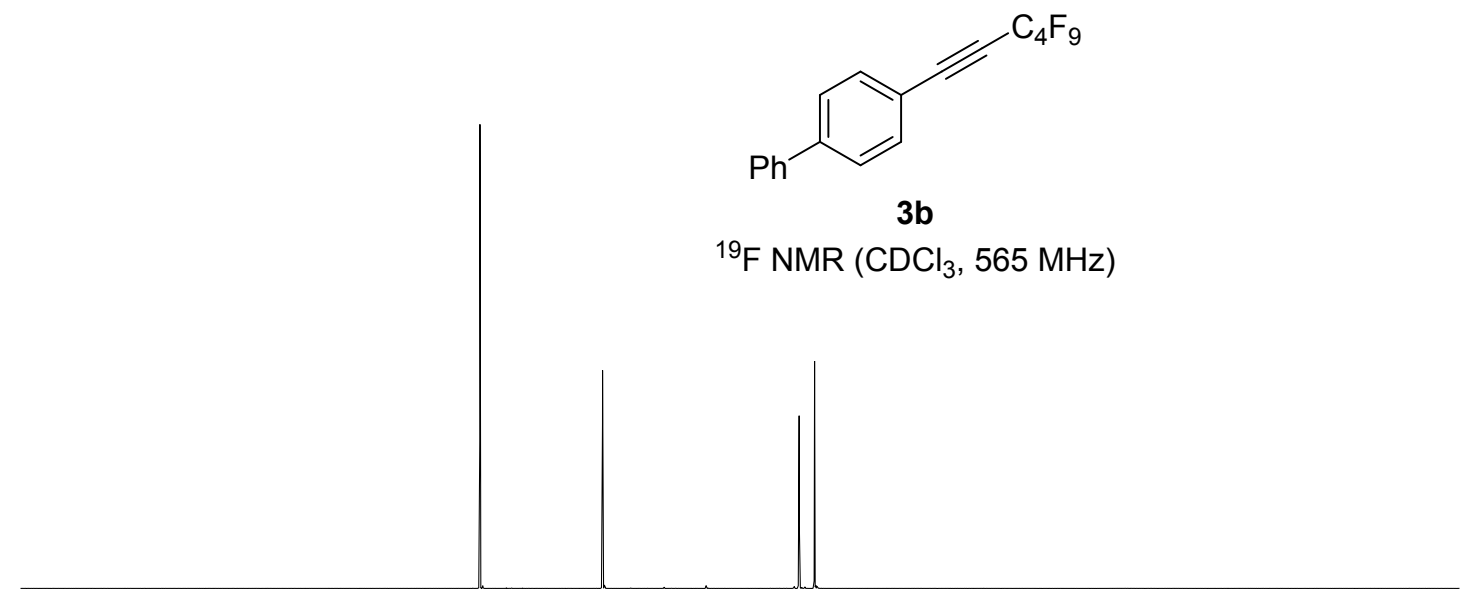

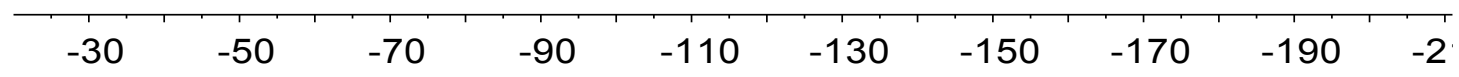




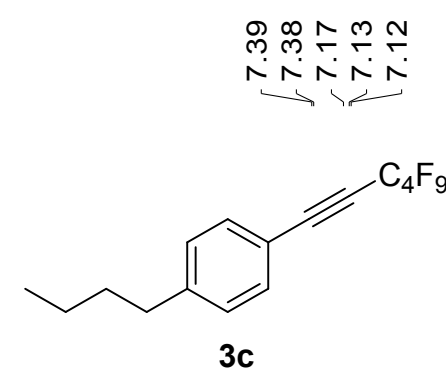

同

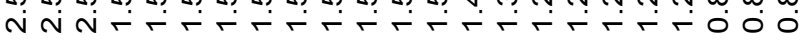

${ }^{1} \mathrm{H} \mathrm{NMR}\left(\mathrm{CDCl}_{3}, 600 \mathrm{MHz}\right)$

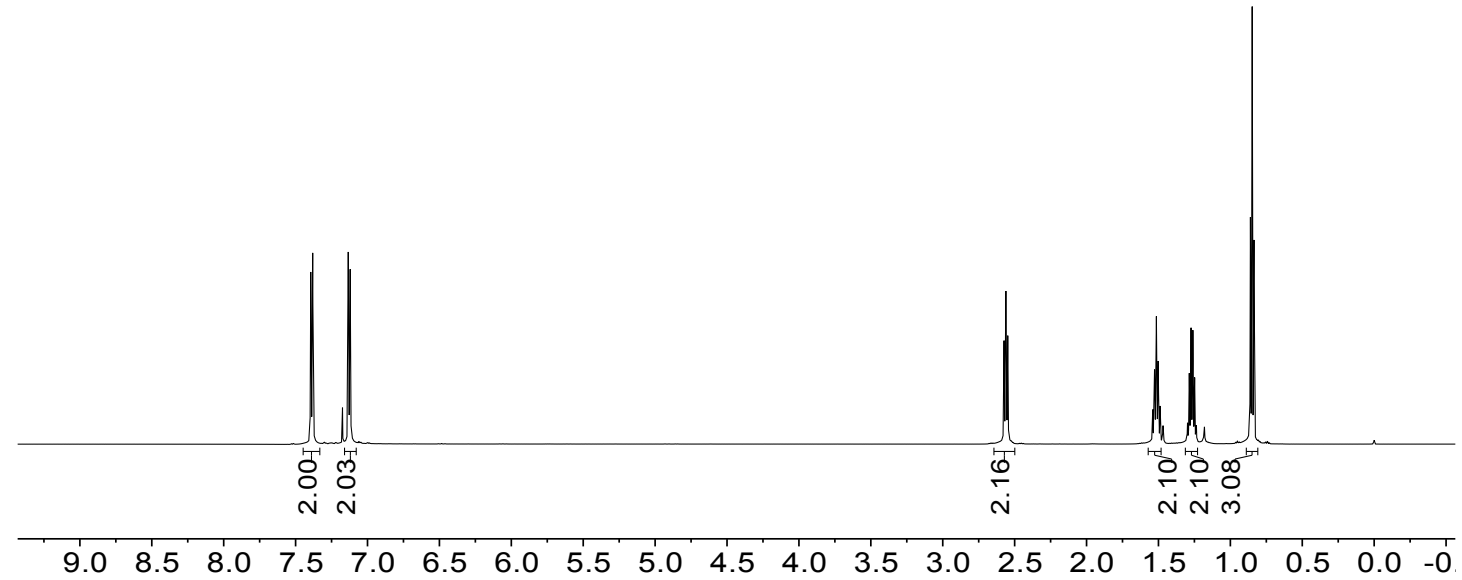

A ○一r

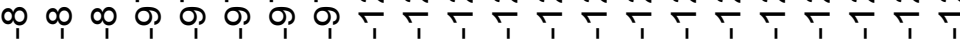

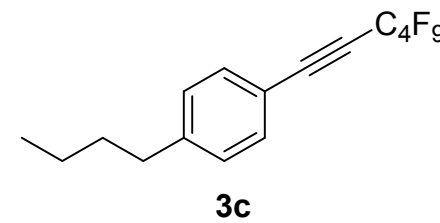

${ }^{19} \mathrm{~F} \mathrm{NMR}\left(\mathrm{CDCl}_{3}, 565 \mathrm{MHz}\right)$

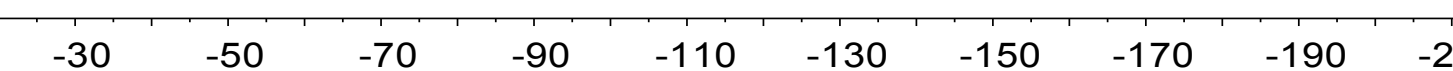


人

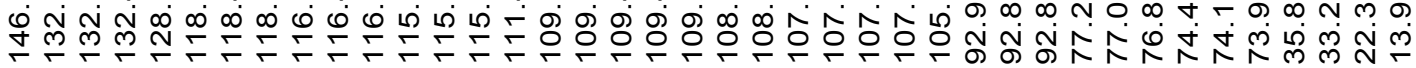

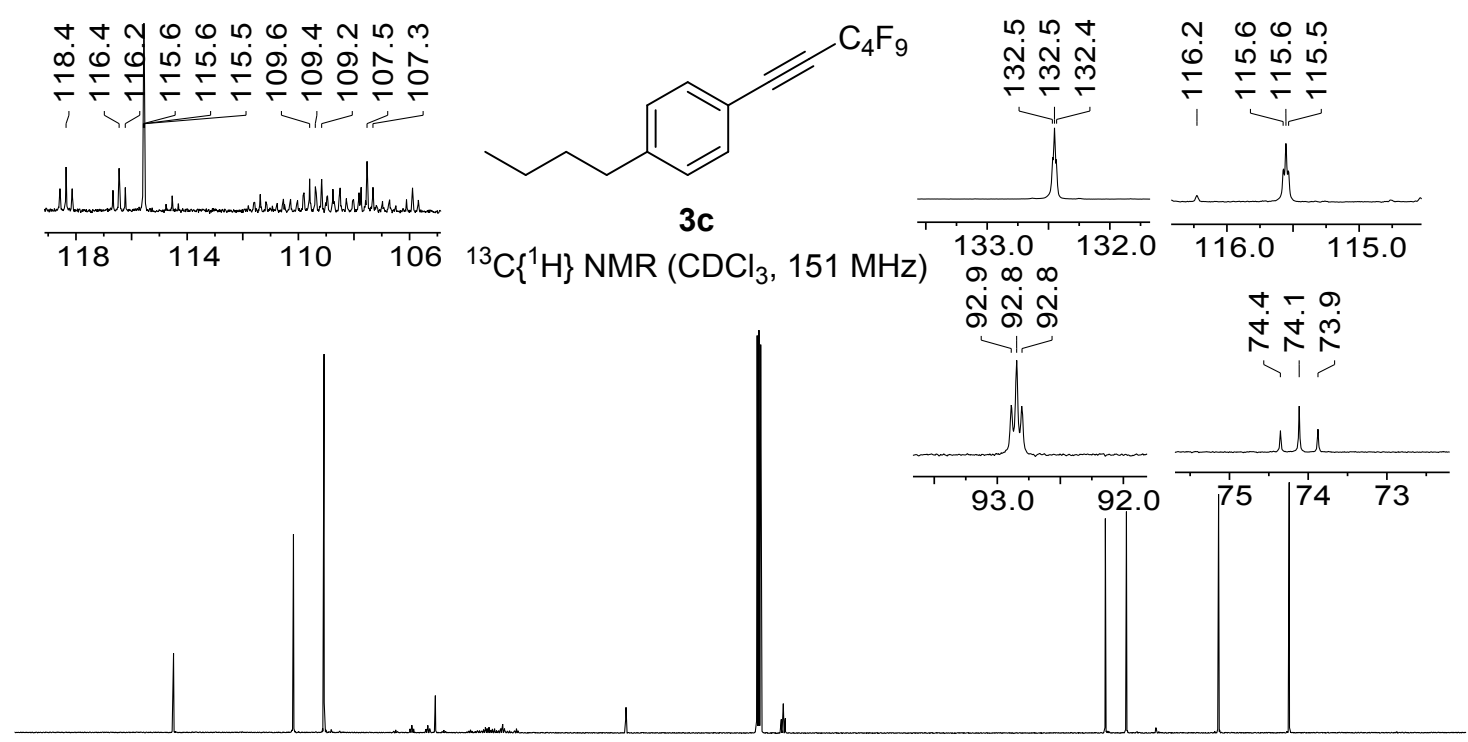

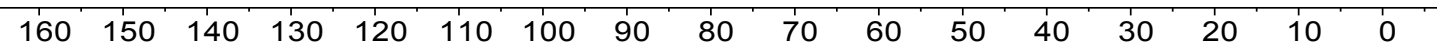

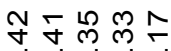

NNNN

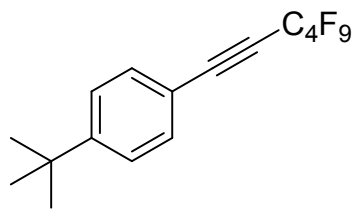

$3 d$

${ }^{1} \mathrm{H} \mathrm{NMR}\left(\mathrm{CDCl}_{3}, 600 \mathrm{MHz}\right)$

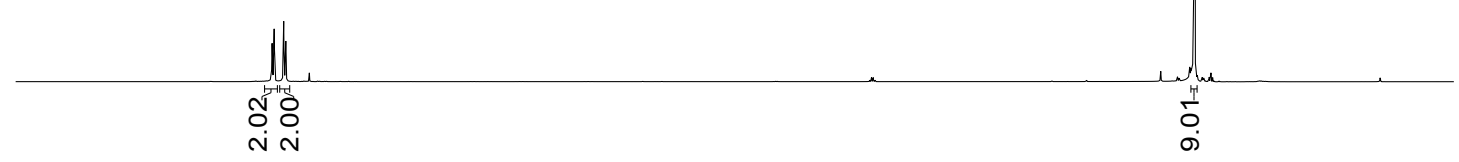

\begin{tabular}{llllllllllllllllllll}
\hline 9.0 & 8.5 & 8.0 & 7.5 & 7.0 & 6.5 & 6.0 & 5.5 & 5.0 & 4.5 & 4.0 & 3.5 & 3.0 & 2.5 & 2.0 & 1.5 & 1.0 & 0.5 & 0.0
\end{tabular} 


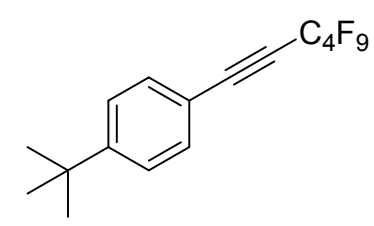

3d

${ }^{19} \mathrm{~F} \mathrm{NMR}\left(\mathrm{CDCl}_{3}, 565 \mathrm{MHz}\right)$

$\begin{array}{llllllllll}-30 & -50 & -70 & -90 & -110 & -130 & -150 & -170 & -190 & -2\end{array}$

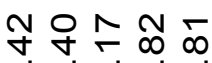

$\hat{N} \wedge 0^{\circ}$

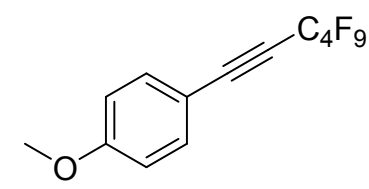

$3 e$

${ }^{1} \mathrm{H} \mathrm{NMR}\left(\mathrm{CDCl}_{3}, 600 \mathrm{MHz}\right)$

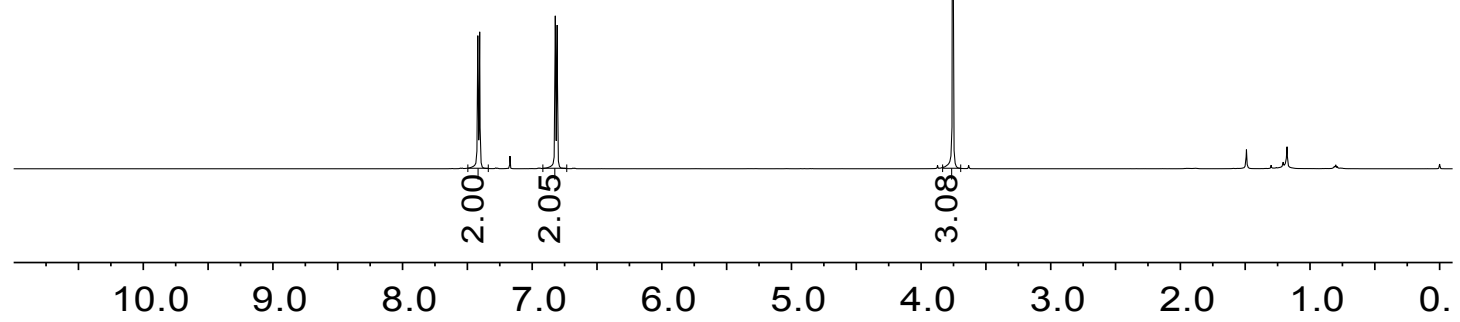




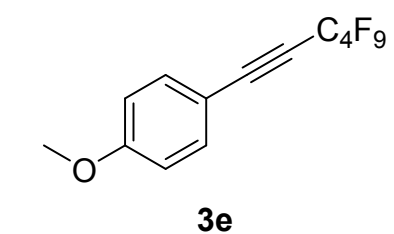

${ }^{19} \mathrm{~F} \mathrm{NMR}\left(\mathrm{CDCl}_{3}, 565 \mathrm{MHz}\right)$
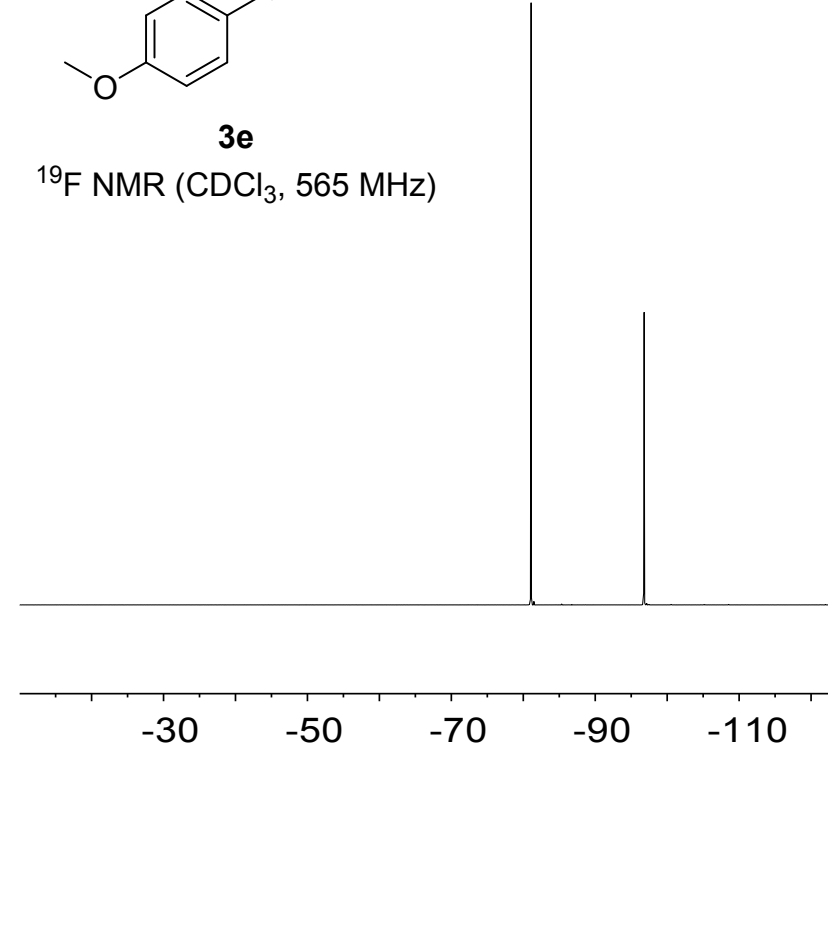

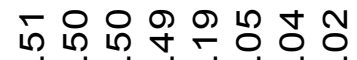

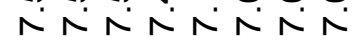

$\begin{array}{lllllllllll}-30 & -50 & -70 & -90 & -110 & -130 & -150 & -170 & -190 & -2\end{array}$

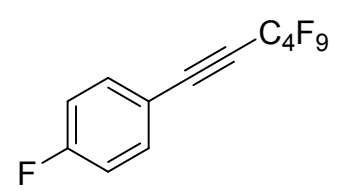

$3 f$

${ }^{1} \mathrm{H} \mathrm{NMR}\left(\mathrm{CDCl}_{3}, 600 \mathrm{MHz}\right)$

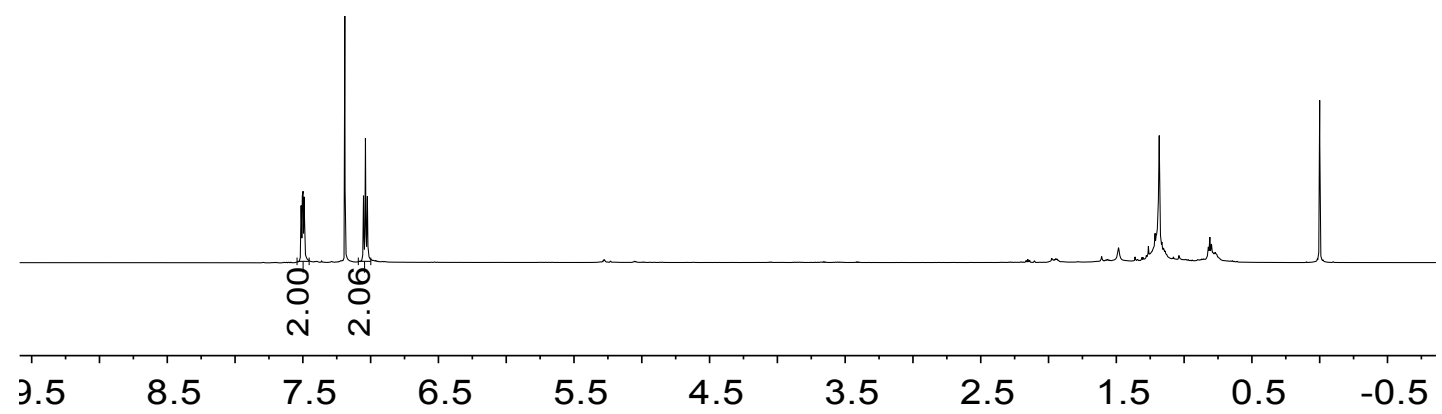




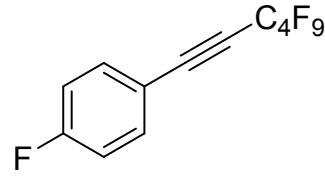

$3 f$

${ }^{19} \mathrm{~F} \mathrm{NMR}\left(\mathrm{CDCl}_{3}, 565 \mathrm{MHz}\right)$

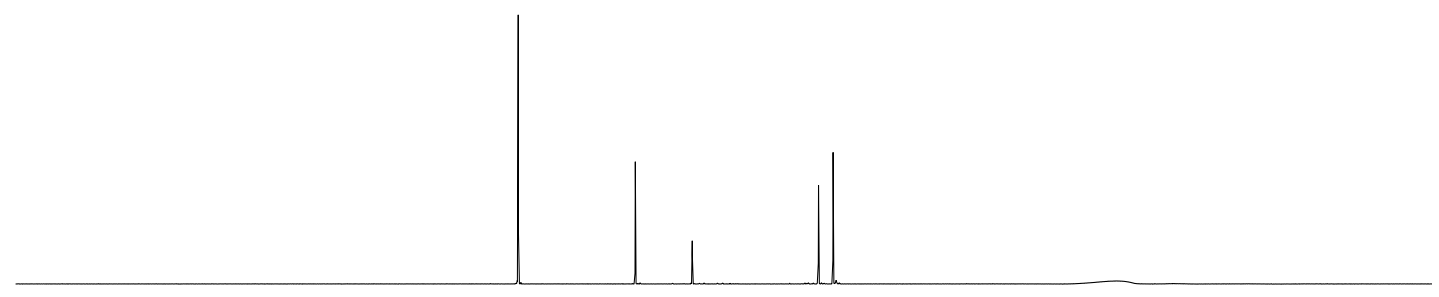

$\begin{array}{llllllllll}-30 & -50 & -70 & -90 & -110 & -130 & -150 & -170 & -190 & -2\end{array}$

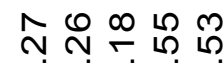

Nกペ

@ִ

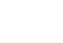

$C^{\mathrm{C}_{4} \mathrm{~F}_{9}}$

$3 g$

${ }^{1} \mathrm{H} \mathrm{NMR}\left(\mathrm{CDCl}_{3}, 600 \mathrm{MHz}\right)$

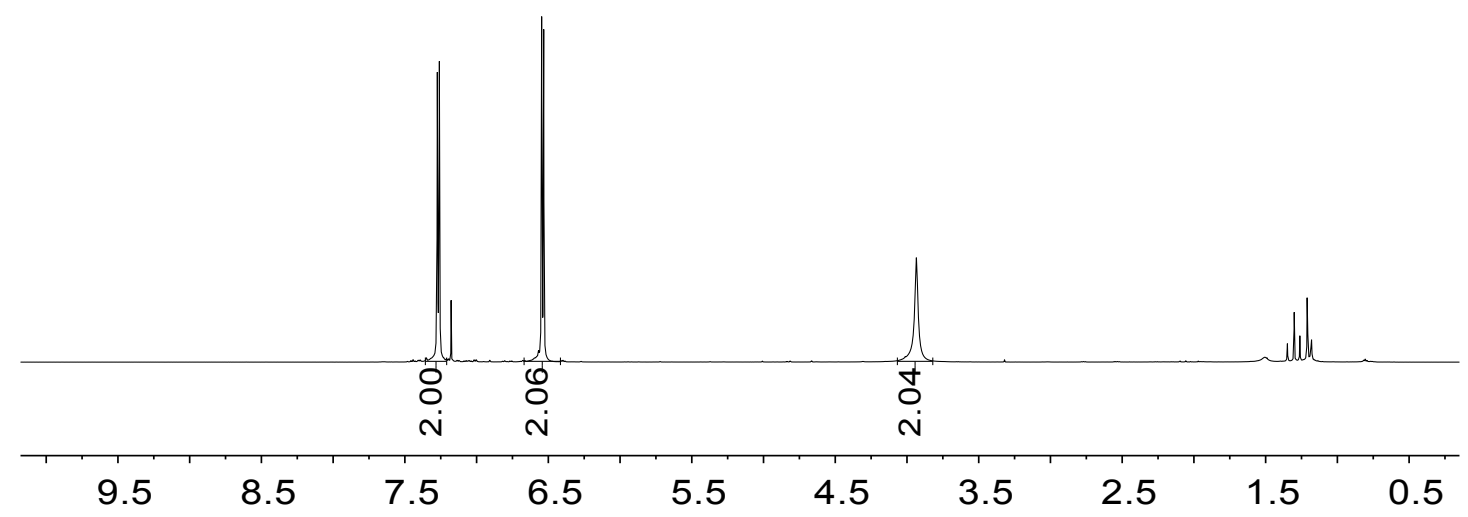




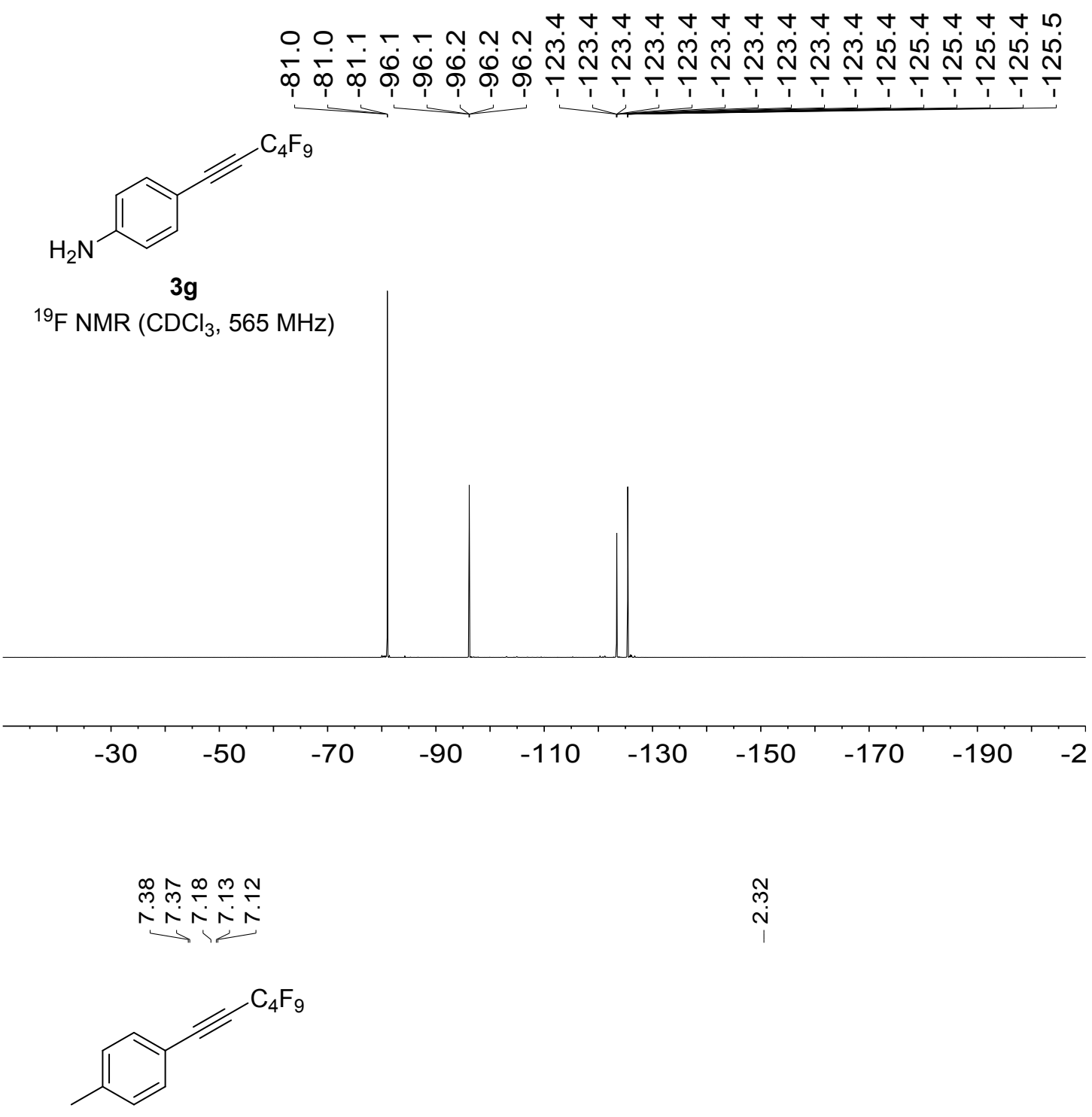

$3 \mathrm{~h}$

${ }^{1} \mathrm{H}$ NMR $\left(\mathrm{CDCl}_{3}, 600 \mathrm{MHz}\right)$

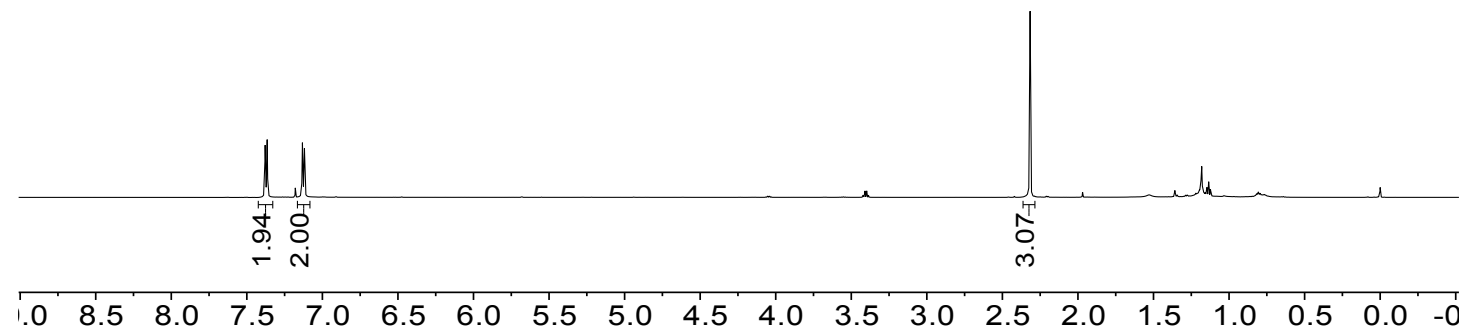




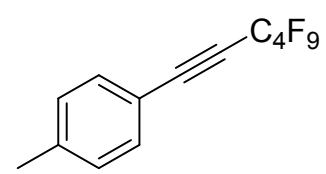

$3 \mathrm{~h}$

${ }^{19} \mathrm{~F}$ NMR $\left(\mathrm{CDCl}_{3}, 565 \mathrm{MHz}\right)$
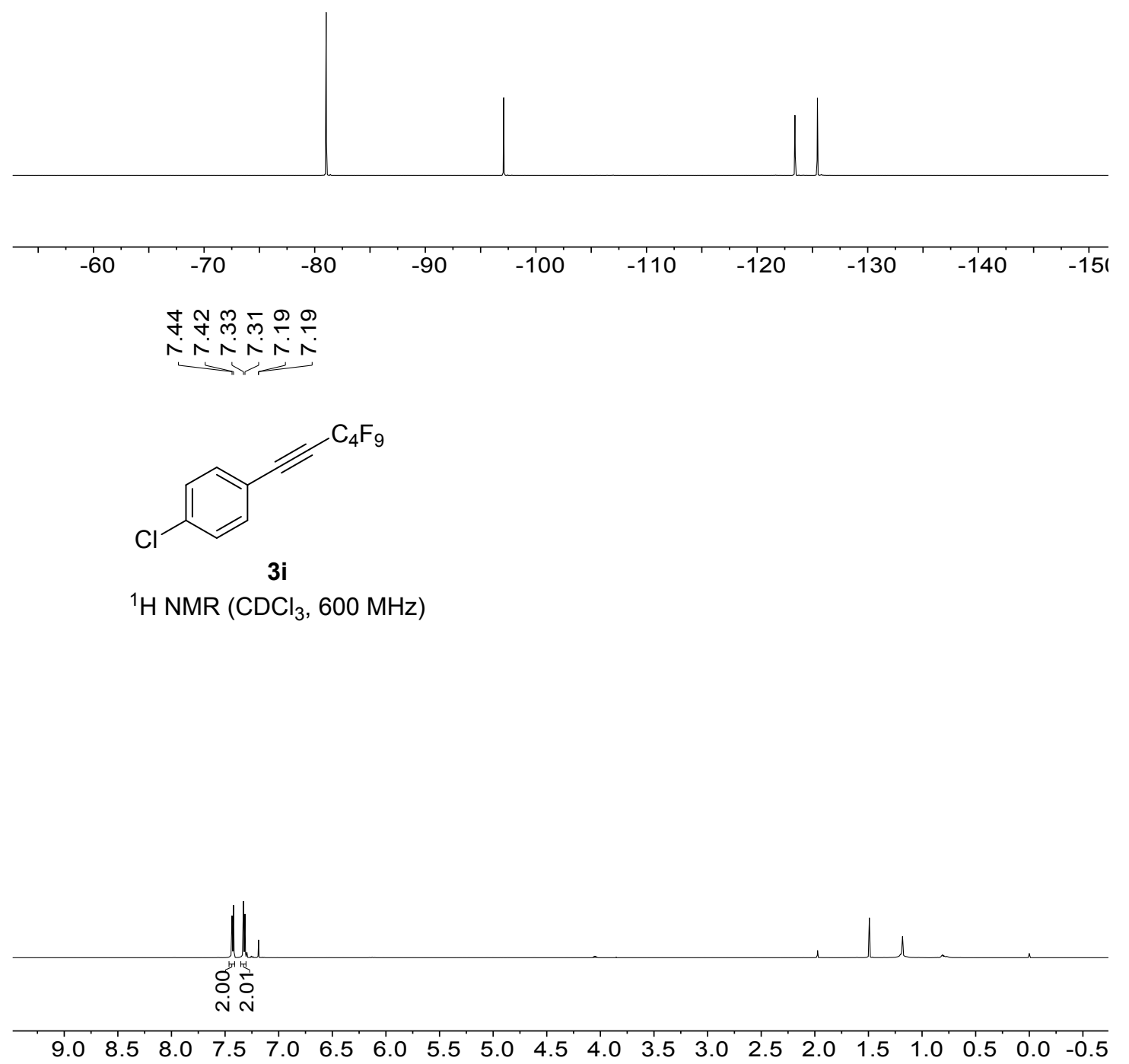


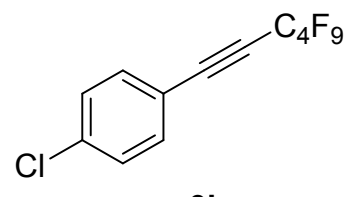

$3 \mathbf{i}$

${ }^{19} \mathrm{~F} \mathrm{NMR}\left(\mathrm{CDCl}_{3}, 565 \mathrm{MHz}\right)$

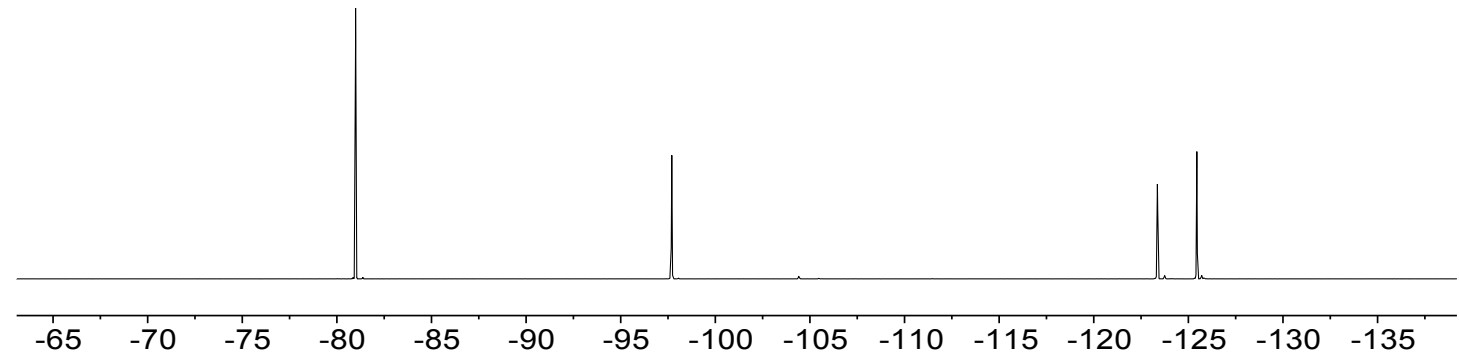

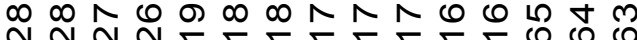

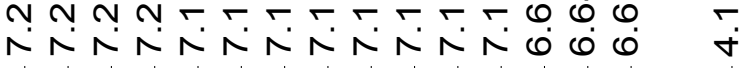<smiles>Nc1ccccc1C#CC(F)(F)F</smiles>

31

${ }^{1} \mathrm{H} \mathrm{NMR}\left(\mathrm{CDCl}_{3}, 600 \mathrm{MHz}\right)$

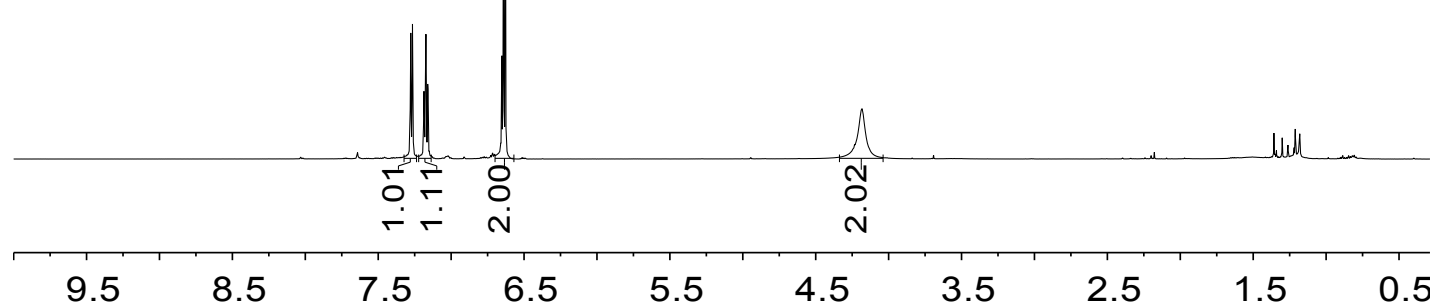




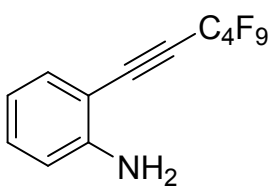

3I

${ }^{19} \mathrm{~F}$ NMR $\left(\mathrm{CDCl}_{3}, 565 \mathrm{MHz}\right)$

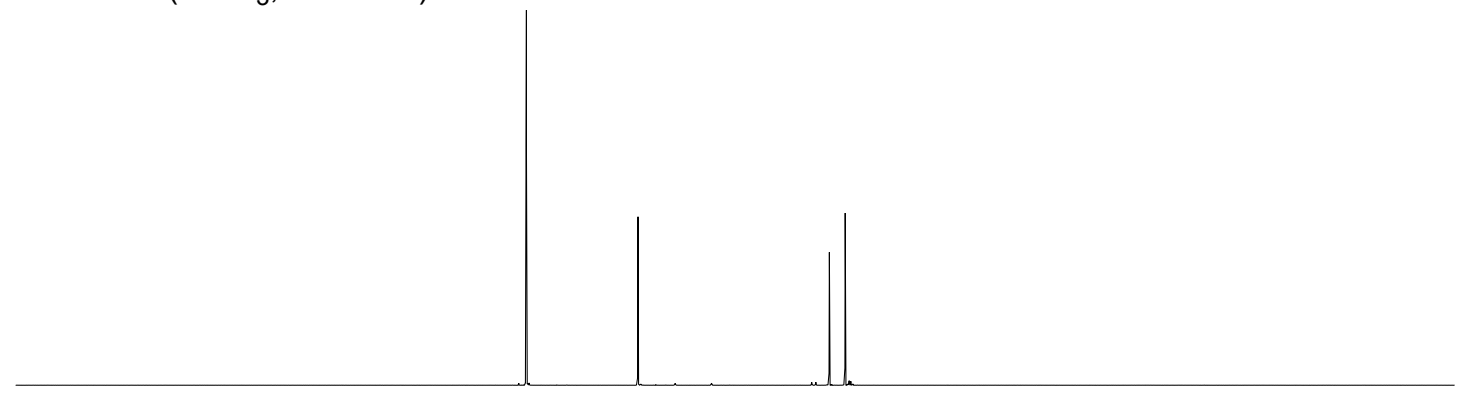

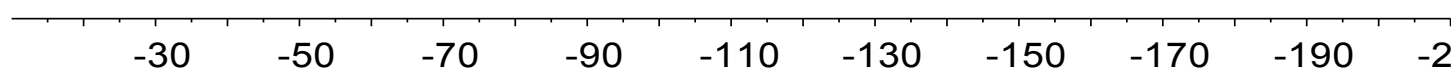

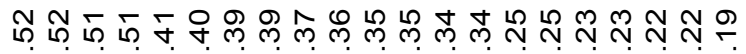

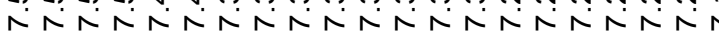

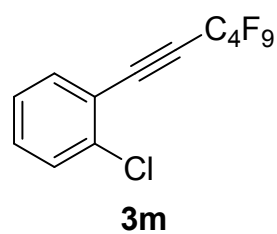

${ }^{1} \mathrm{H} \mathrm{NMR}\left(\mathrm{CDCl}_{3}, 600 \mathrm{MHz}\right)$

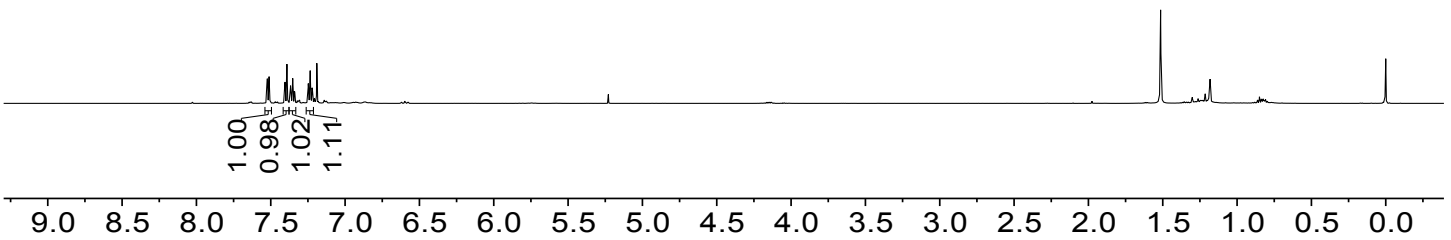




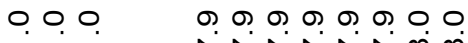

চ

1<smiles>FC(F)(F)C#Cc1ccccc1Cl</smiles>

$3 m$

${ }^{19} \mathrm{~F} \mathrm{NMR}\left(\mathrm{CDCl}_{3}, 565 \mathrm{MHz}\right)$

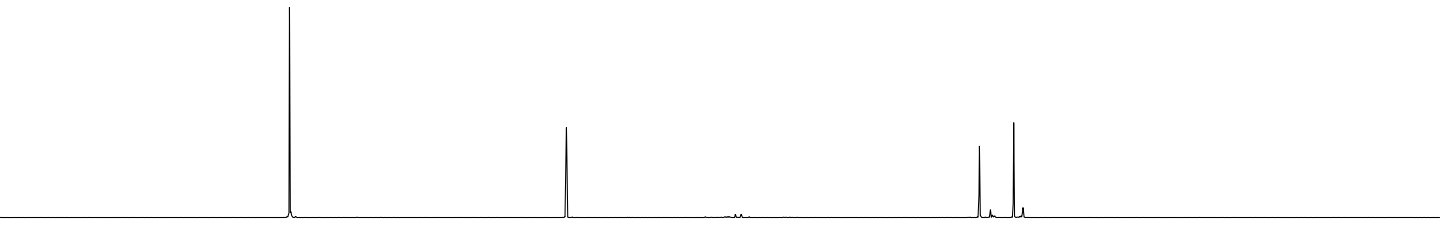

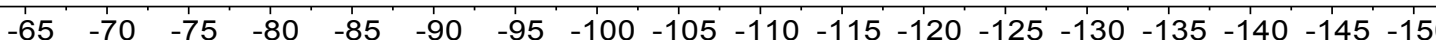

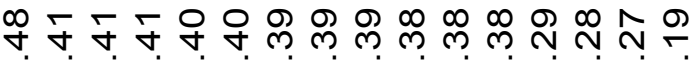

N

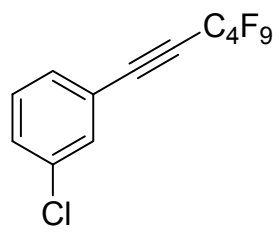

$3 n$

${ }^{1} \mathrm{H} \mathrm{NMR}\left(\mathrm{CDCl}_{3}, 600 \mathrm{MHz}\right)$

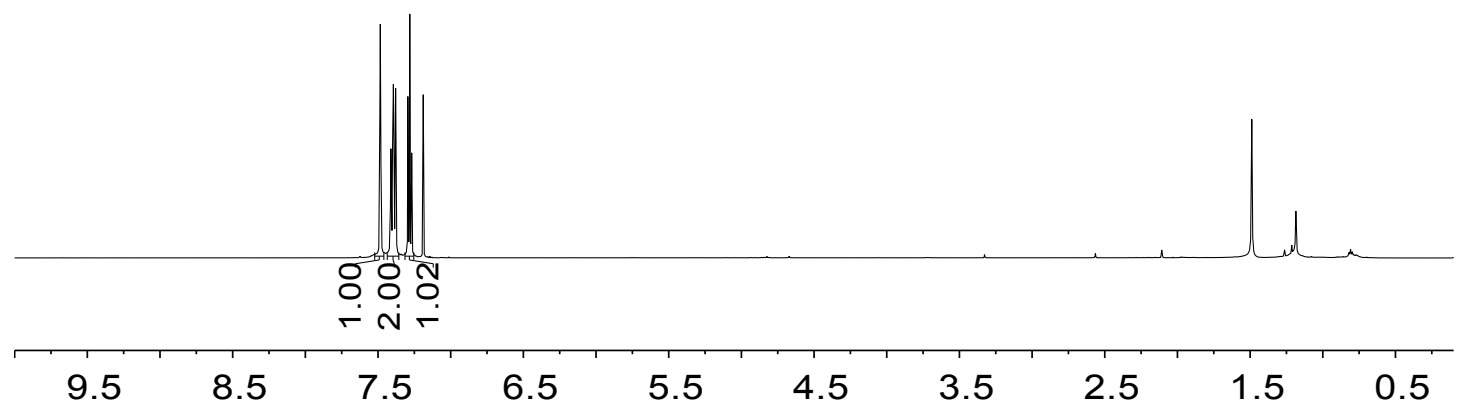




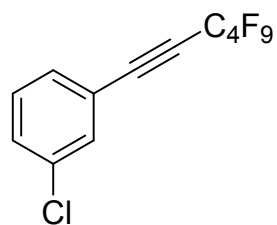

$3 n$

${ }^{19} \mathrm{~F} \mathrm{NMR}\left(\mathrm{CDCl}_{3}, 565 \mathrm{MHz}\right)$

$\begin{array}{llllllllll}-30 & -50 & -70 & -90 & -110 & -130 & -150 & -170 & -190 & -2\end{array}$

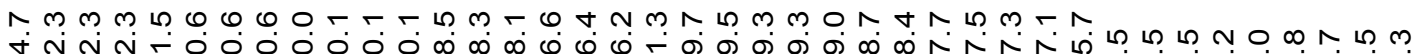
崩

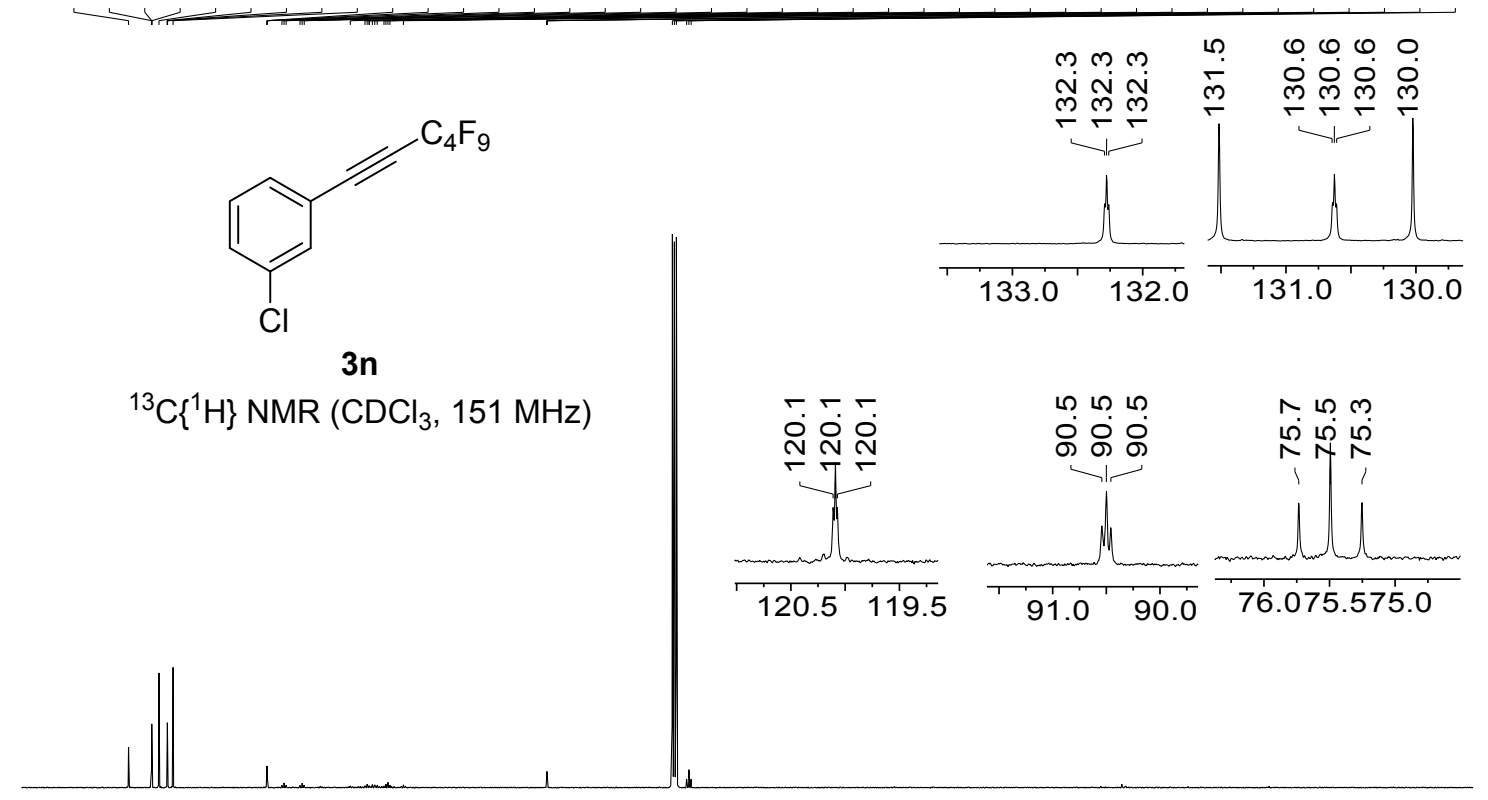

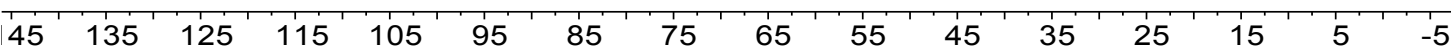




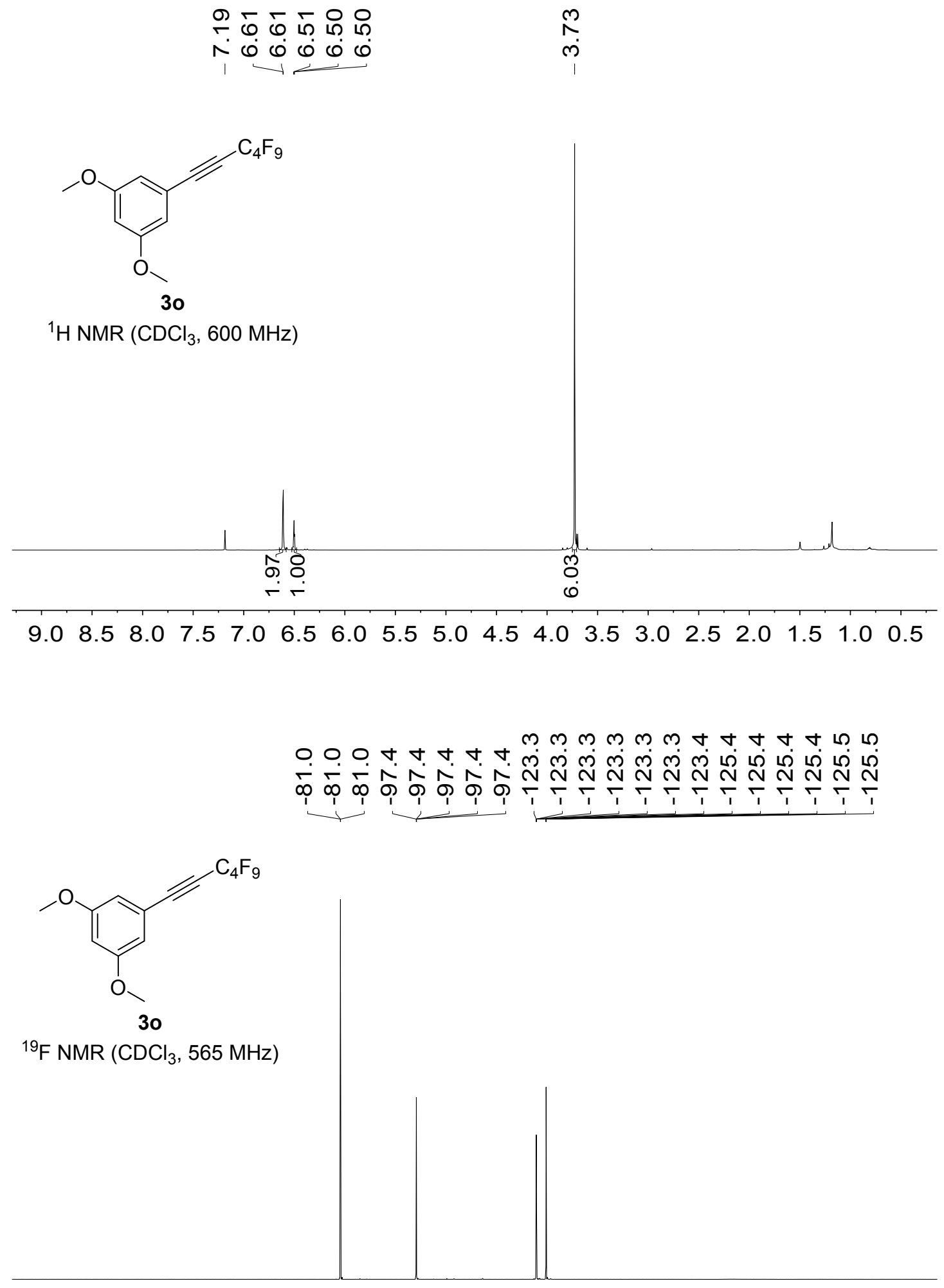

\begin{tabular}{llllllllll}
\hline-30 & -50 & -70 & -90 & -110 & -130 & -150 & -170 & -190 & -2
\end{tabular}




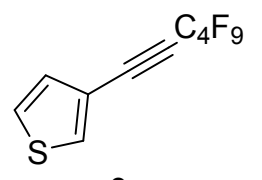

$3 p$

${ }^{1} \mathrm{H} \mathrm{NMR}\left(\mathrm{CDCl}_{3}, 600 \mathrm{MHz}\right)$

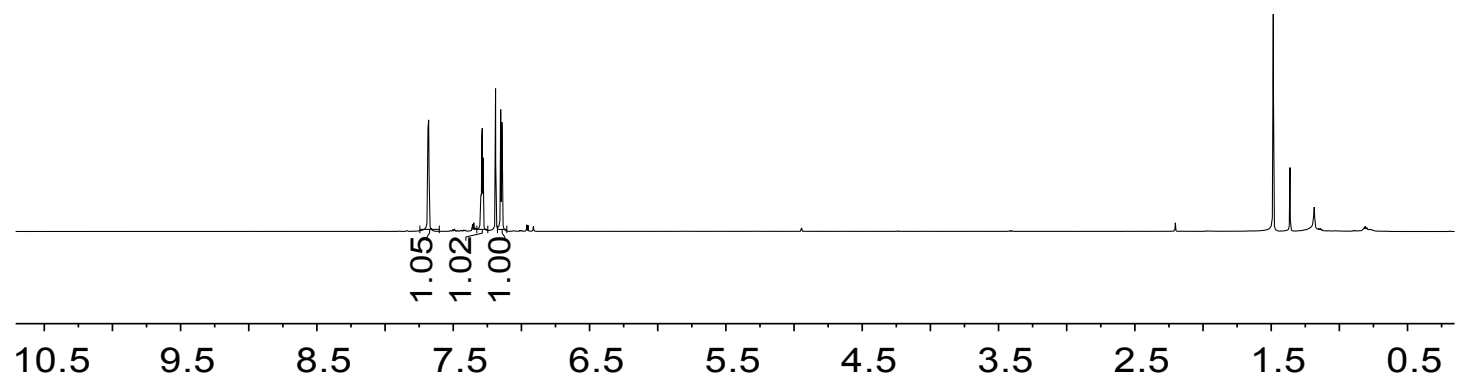

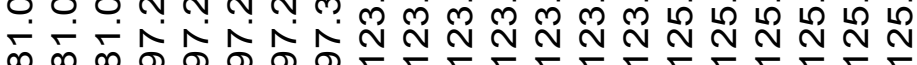

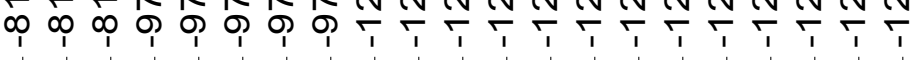

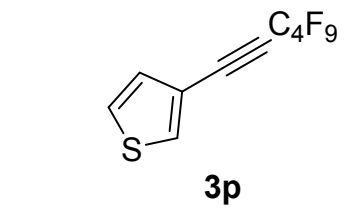

${ }^{19} \mathrm{~F} \mathrm{NMR}\left(\mathrm{CDCl}_{3}, 565 \mathrm{MHz}\right)$

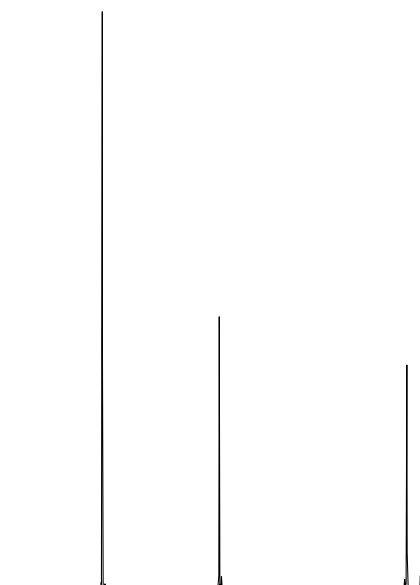

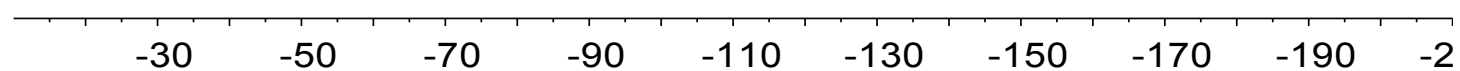




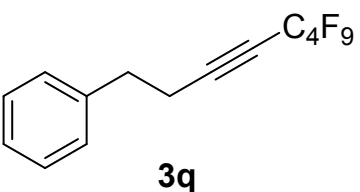

$3 q$

${ }^{1} \mathrm{H} \mathrm{NMR}\left(\mathrm{CDCl}_{3}, 600 \mathrm{MHz}\right)$

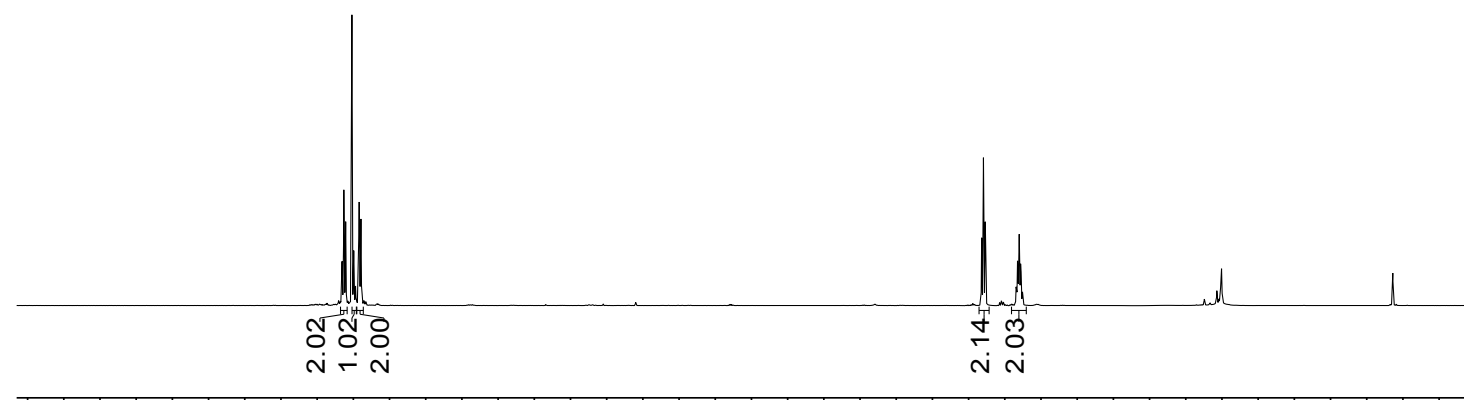

\begin{tabular}{lllllllllllllllllllllllll}
\hline 9.5 & 9.0 & 8.5 & 8.0 & 7.5 & 7.0 & 6.5 & 6.0 & 5.5 & 5.0 & 4.5 & 4.0 & 3.5 & 3.0 & 2.5 & 2.0 & 1.5 & 1.0 & 0.5 & 0.0
\end{tabular}

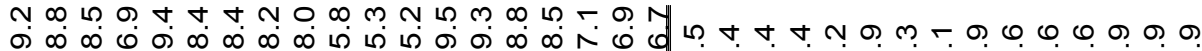

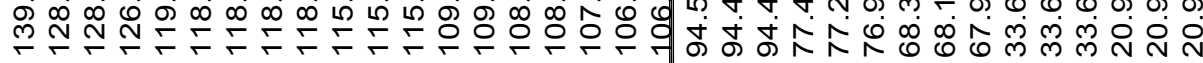

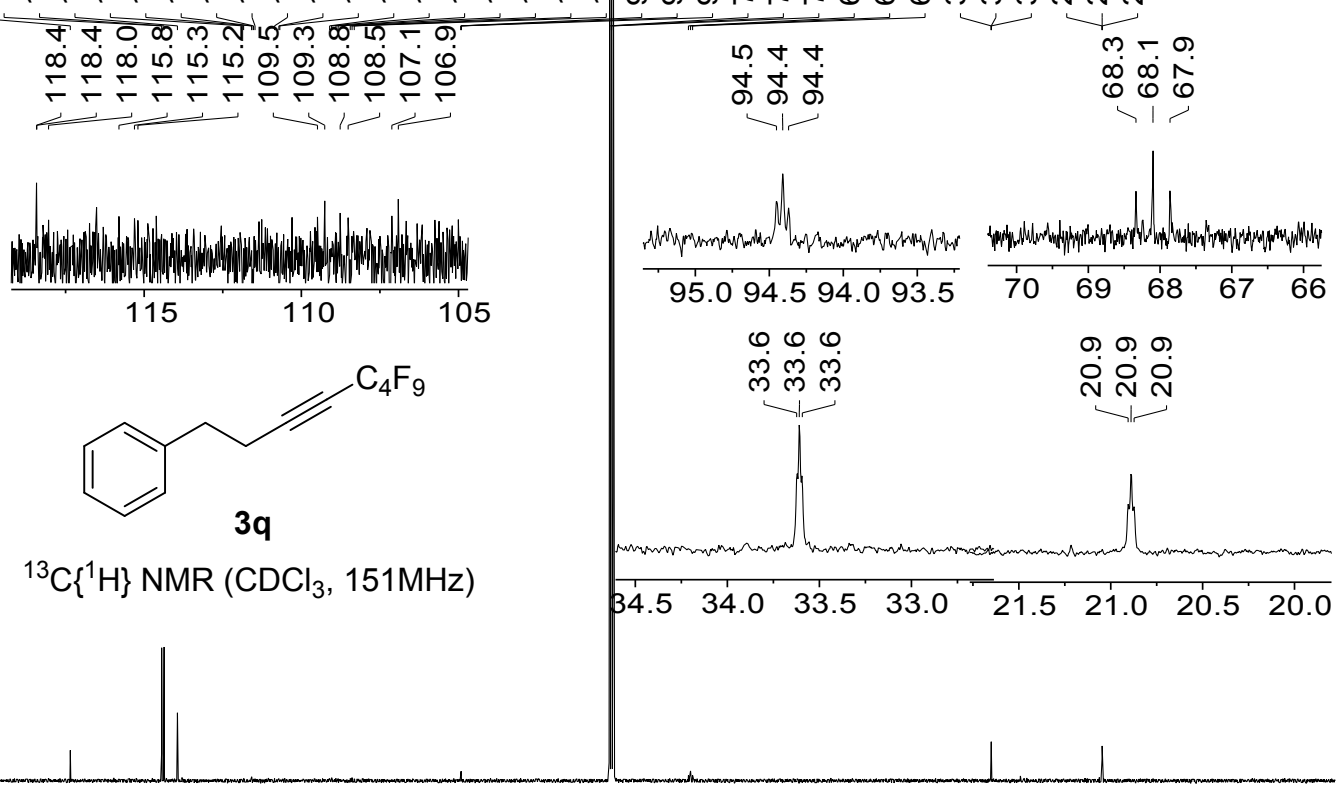

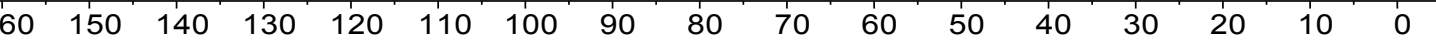




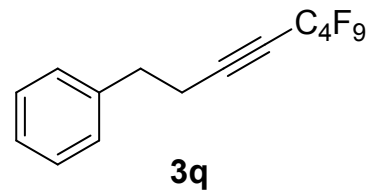

${ }^{19} \mathrm{~F} \mathrm{NMR}\left(\mathrm{CDCl}_{3}, 565 \mathrm{MHz}\right)$

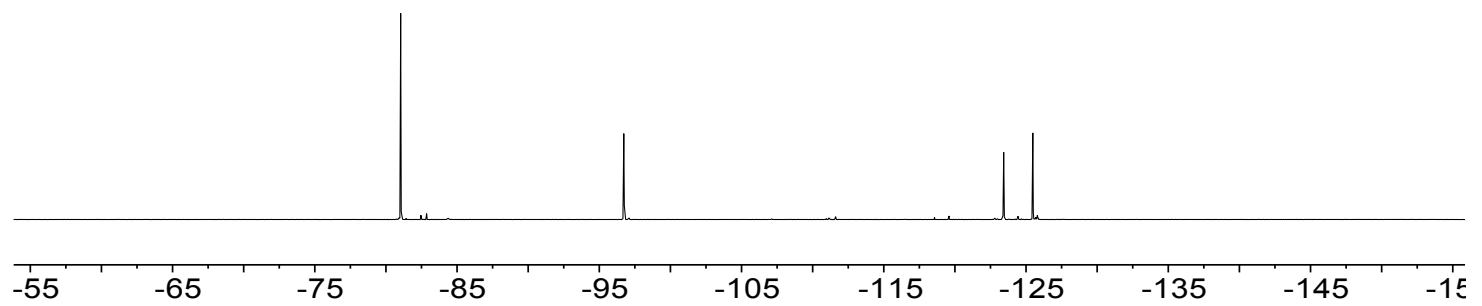

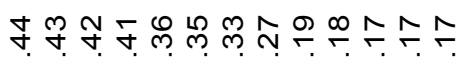

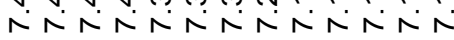

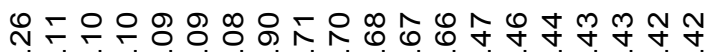

min<smiles>C#CC(C)C(=O)N(C)c1ccccc1</smiles>

$1 \mathrm{r}$

${ }^{1} \mathrm{H} \mathrm{NMR}\left(\mathrm{CDCl}_{3}, 600 \mathrm{MHz}\right)$

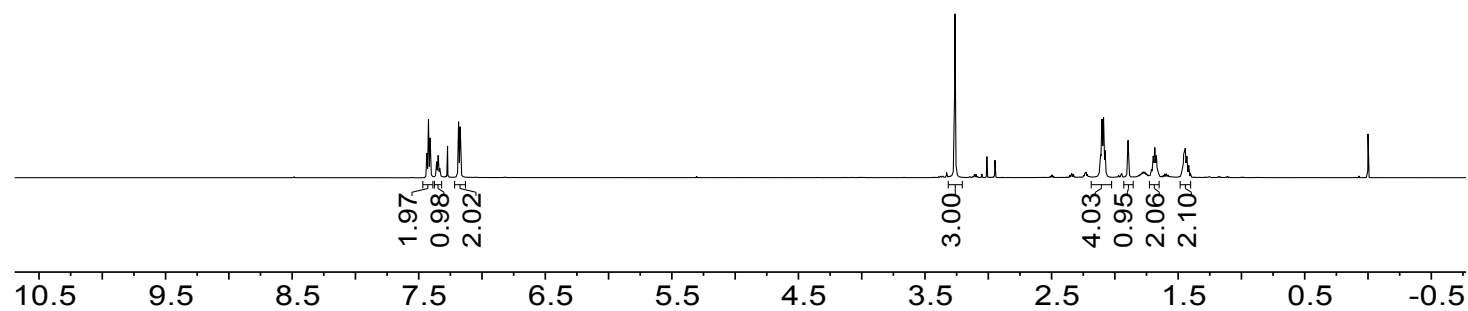




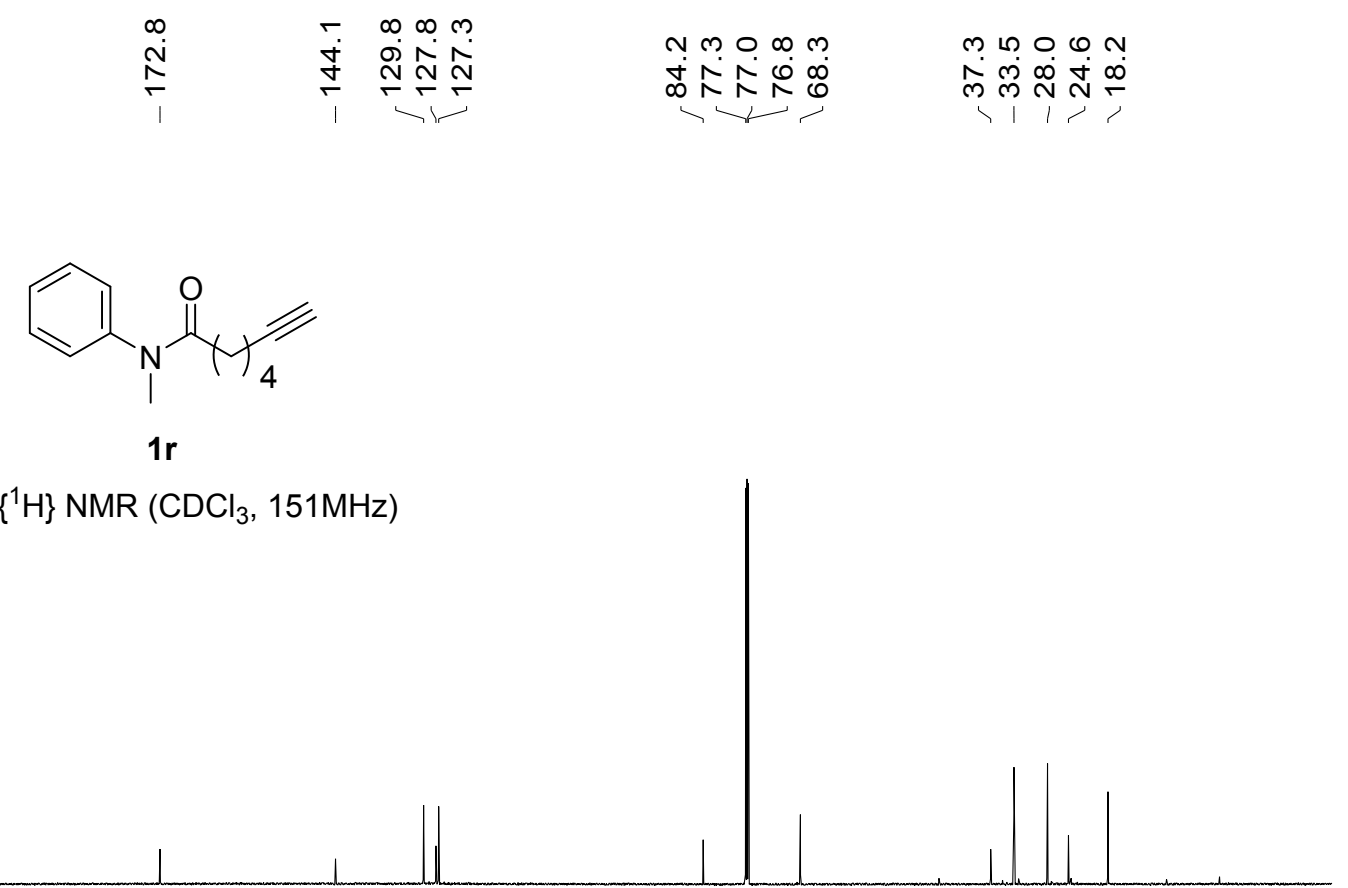

$21020019018017016015014013012011010090 \quad 8070 \quad 60 \begin{array}{lllllllll}1 & 40 & 30 & 20 & 10 & 0 & -10\end{array}$

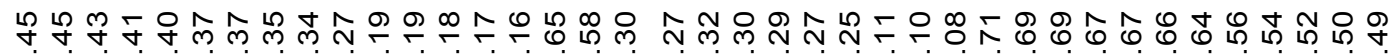

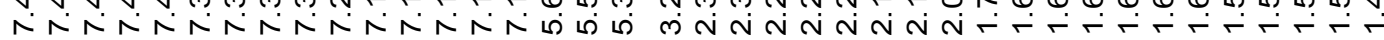<smiles>CC(C#CCC(F)(F)F)C(=O)N(C)c1ccccc1</smiles>

$3 r$

${ }^{1} \mathrm{H}$ NMR $\left(\mathrm{CDCl}_{3}, 400 \mathrm{MHz}\right)$

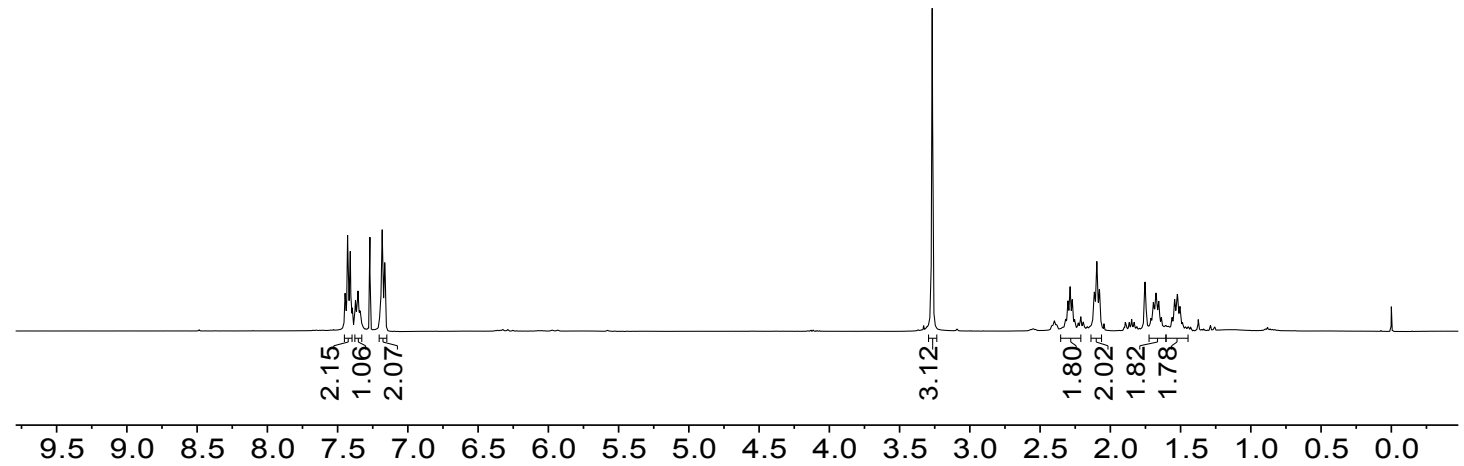


<smiles>CN(C(=O)C#CC(F)(F)F)c1ccccc1</smiles>

$3 r$

${ }^{19} \mathrm{~F} \mathrm{NMR}\left(\mathrm{CDCl}_{3}, 565 \mathrm{MHz}\right)$

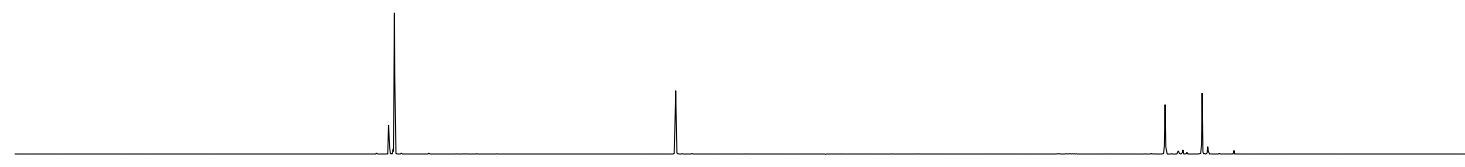

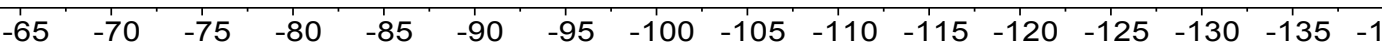

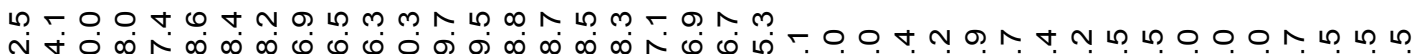

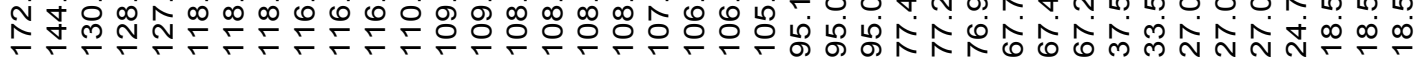

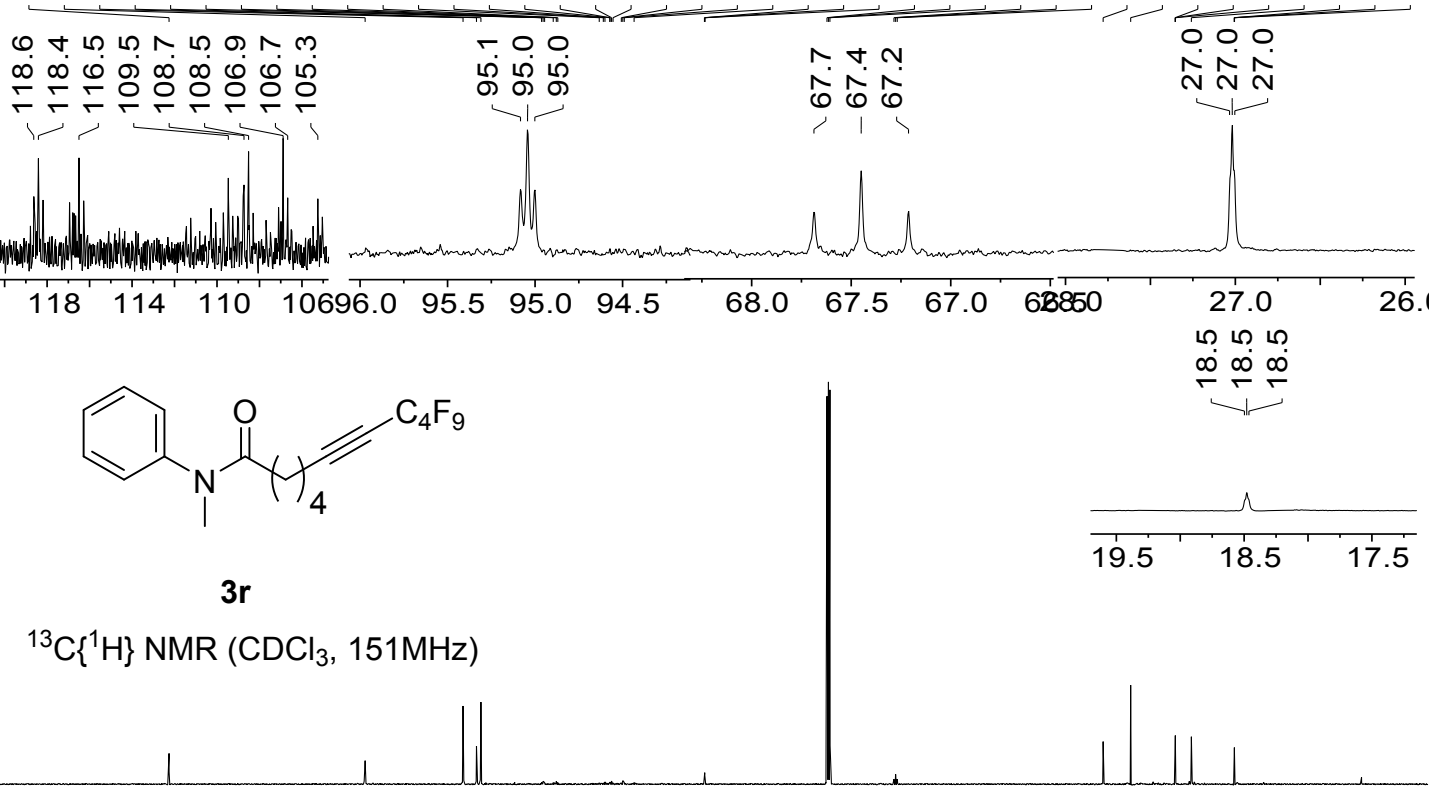

\begin{tabular}{llllllllllllllllllllll}
\hline 00 & 190 & 180 & 170 & 160 & 150 & 140 & 130 & 120 & 110 & 100 & 90 & 80 & 70 & 60 & 50 & 40 & 30 & 20 & 10 & 0
\end{tabular} 


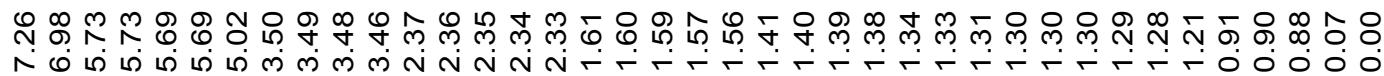

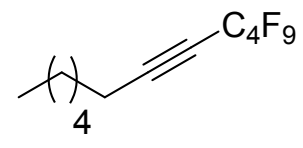

3s

${ }^{1} \mathrm{H} \mathrm{NMR}\left(\mathrm{CDCl}_{3}, 600 \mathrm{MHz}\right)$
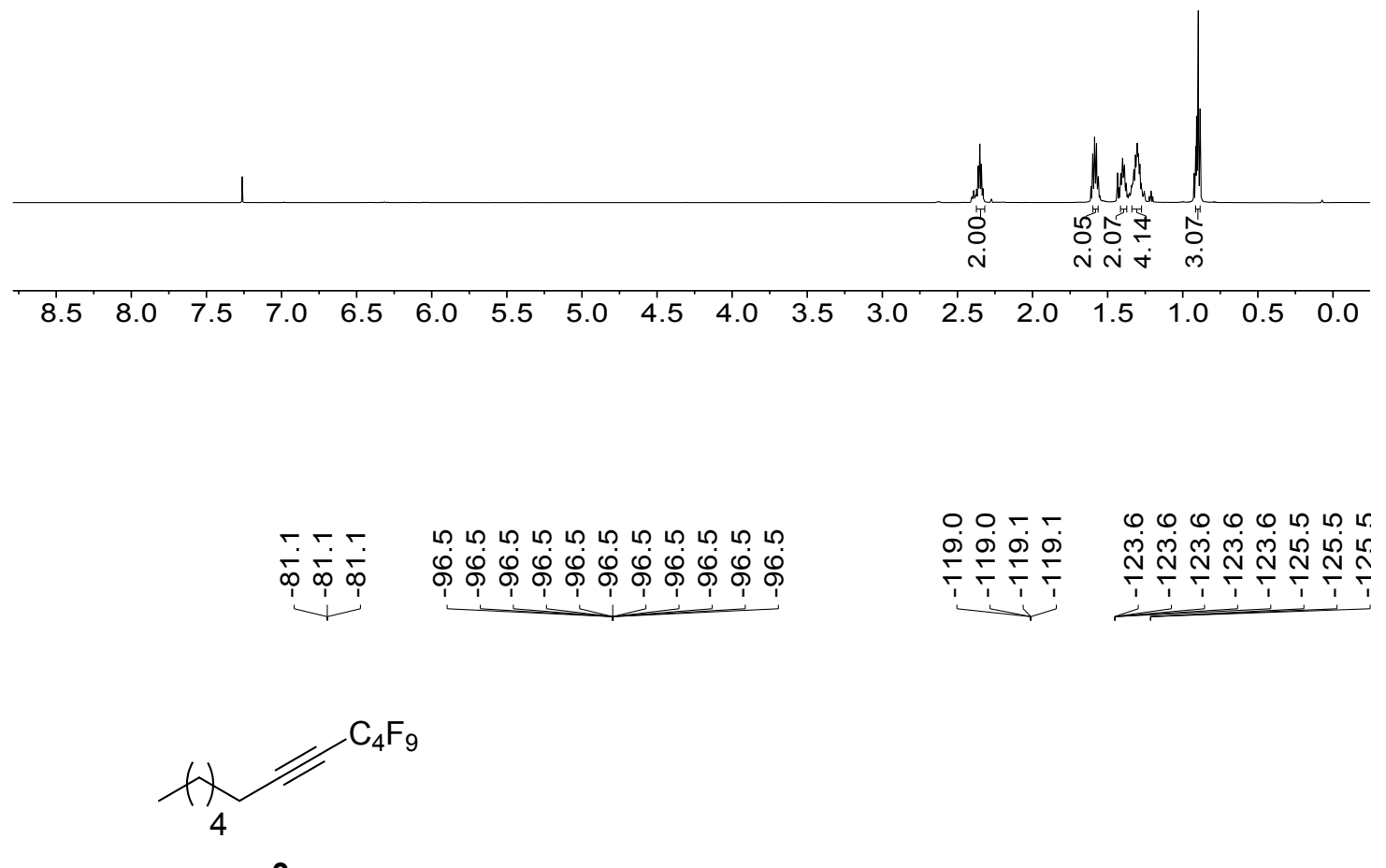

3s

${ }^{1} \mathrm{H} \mathrm{NMR}\left(\mathrm{CDCl}_{3}, 600 \mathrm{MHz}\right)$

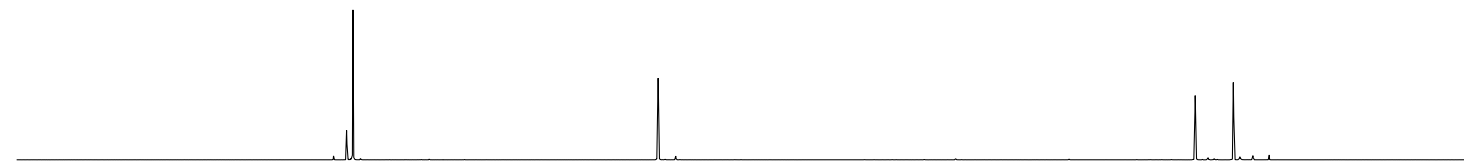

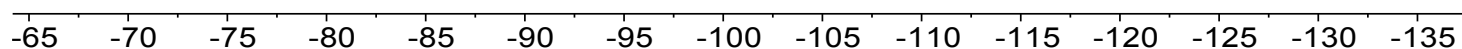




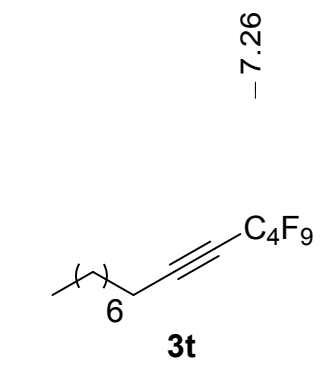

m

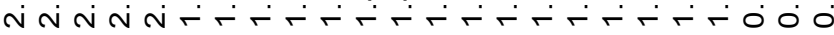

${ }^{1} \mathrm{H} \mathrm{NMR}\left(\mathrm{CDCl}_{3}, 600 \mathrm{MHz}\right)$

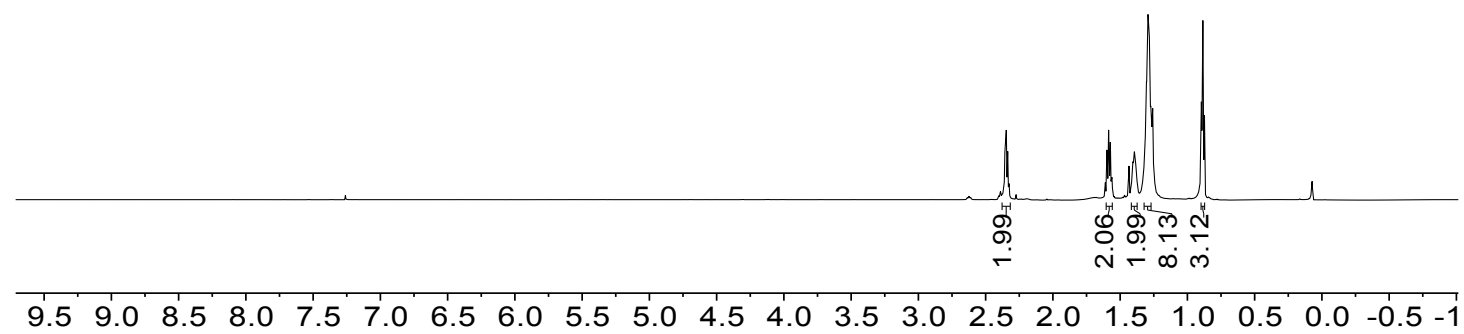

N $N$

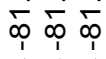

r

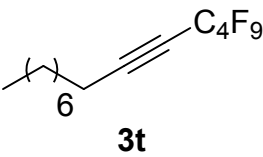

${ }^{19} \mathrm{~F} \mathrm{NMR}\left(\mathrm{CDCl}_{3}, 565 \mathrm{MHz}\right)$

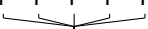

กิบ

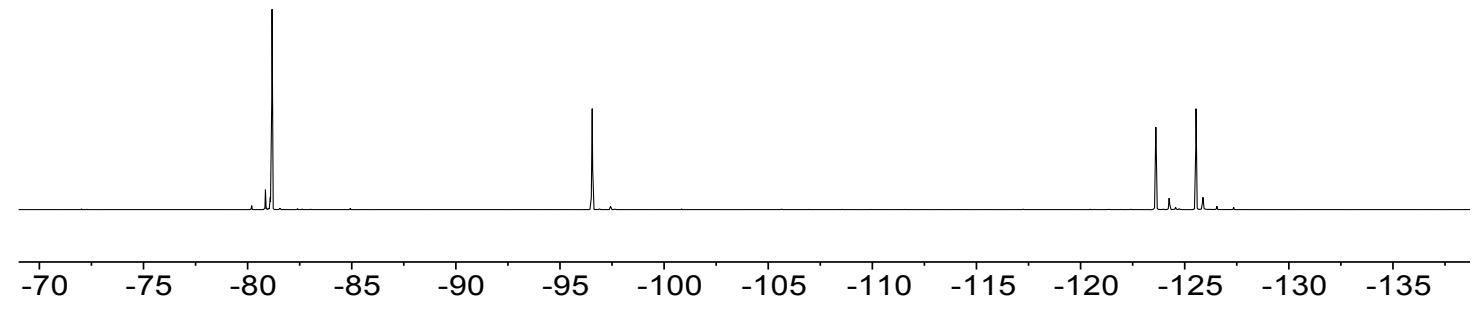


人 車

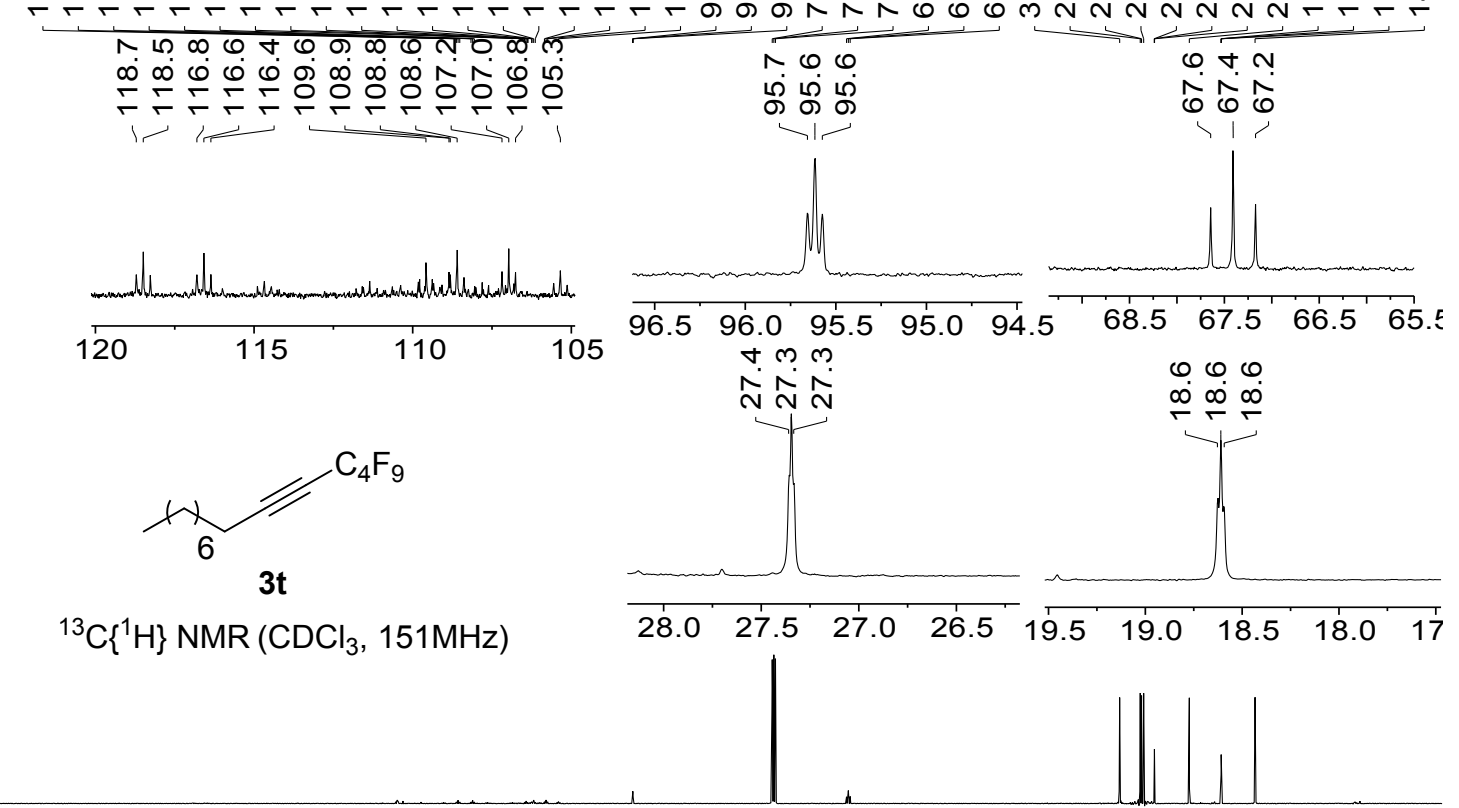

$\begin{array}{lllllllllllllllllll}1 & 160 & 150 & 140 & 130 & 120 & 110 & 100 & 90 & 80 & 70 & 60 & 50 & 40 & 30 & 20 & 10 & 0 & -1\end{array}$

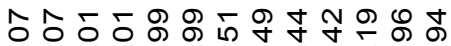

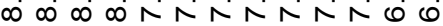<smiles>C#Cc1ccc(NC(=O)c2sc(-c3ccc(OCC(C)C)c(C#N)c3)nc2C)cc1</smiles>

Febuxostat-tethered alkyne

${ }^{1} \mathrm{H}$ NMR $\left(\mathrm{CDCl}_{3}, 400 \mathrm{MHz}\right)$

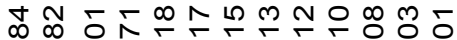

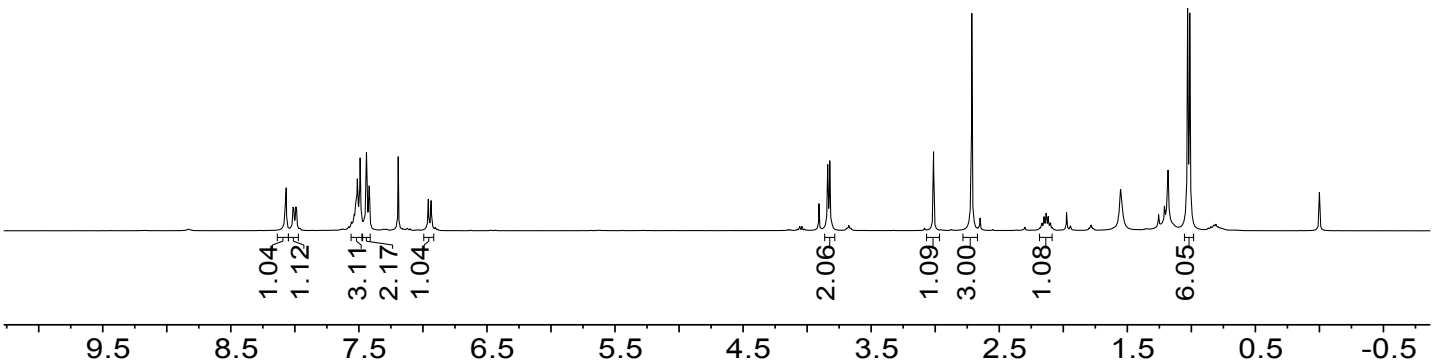




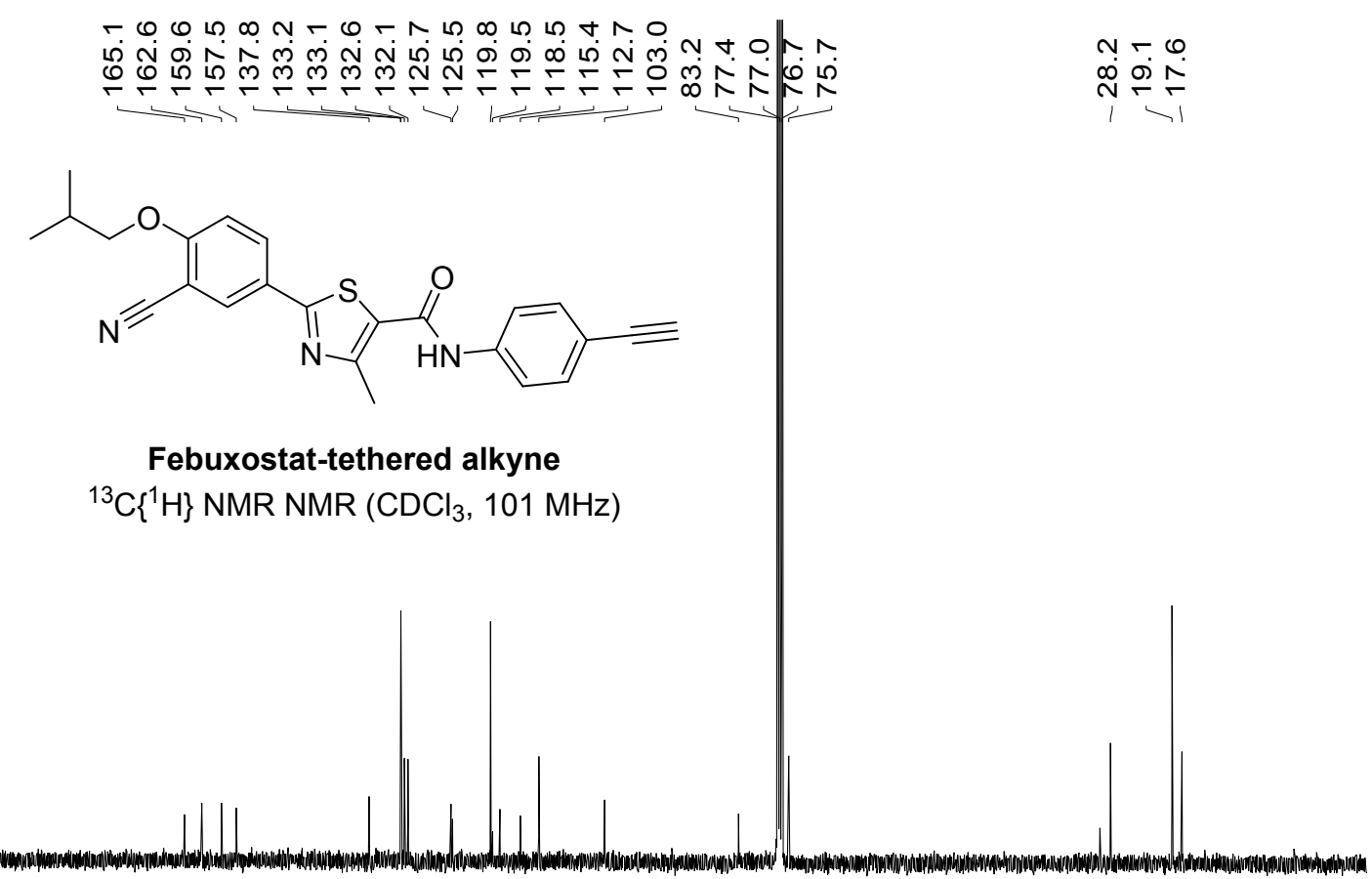

$\begin{array}{llllllllllllllllllll}190 & 180 & 170 & 160 & 150 & 140 & 130 & 120 & 110 & 100 & 90 & 80 & 70 & 60 & 50 & 40 & 30 & 20 & 10 & 0\end{array}$

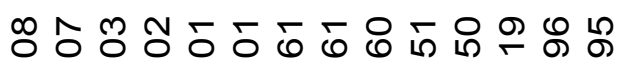

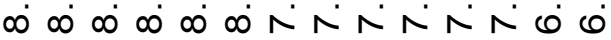<smiles>Cc1nc(-c2ccc(OCC(C)C)c(C#N)c2)sc1C(=O)Nc1ccc(C#CC(F)(F)F)cc1</smiles>

$3 \mathbf{u}$

${ }^{1} \mathrm{H} \mathrm{NMR}\left(\mathrm{CDCl}_{3}, 600 \mathrm{MHz}\right)$

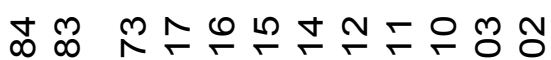

m

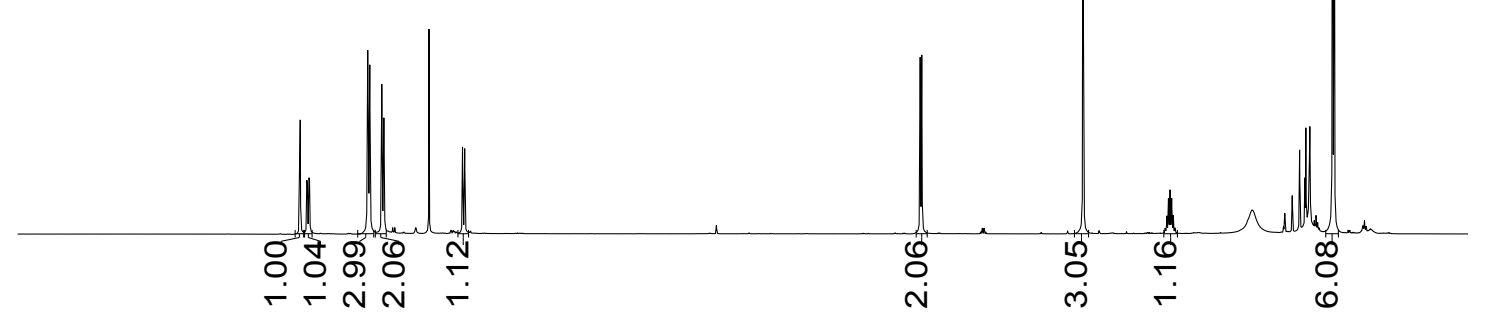

$9.59 .08 .58 .07 .57 .06 .56 .05 .5 \quad 5.04 .54 .03 .53 .02 .52 .01 .51 .0 \quad 0.5$ 
$\begin{array}{rrr}9 & 0 \\ 0 & 0 \\ 0 & 0 \\ 0 & 0 \\ 1 & 1 & 0 \\ 1 & 1\end{array}$

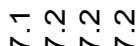

कें क़ि क़

$m m m m m m+t \nabla t \theta$

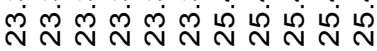

$\div \div \div \div \div \div \div$<smiles>Cc1nc(-c2ccc(OCC(C)C)c(C#N)c2)sc1C(=O)Nc1ccc(C#CC(F)F)cc1</smiles>

$3 \mathbf{u}$

${ }^{19} \mathrm{~F} \mathrm{NMR}\left(\mathrm{CDCl}_{3}, 565 \mathrm{MHz}\right)$

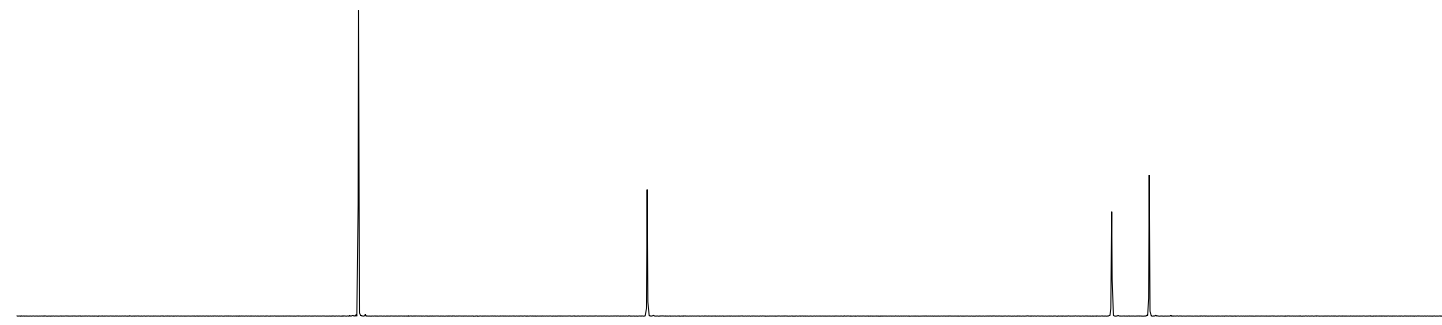

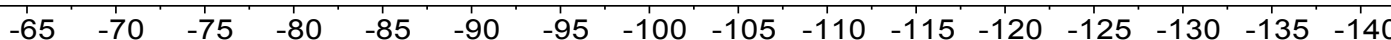

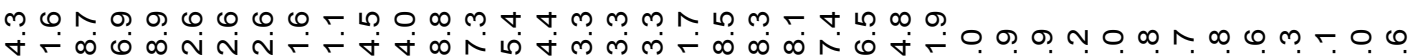
过
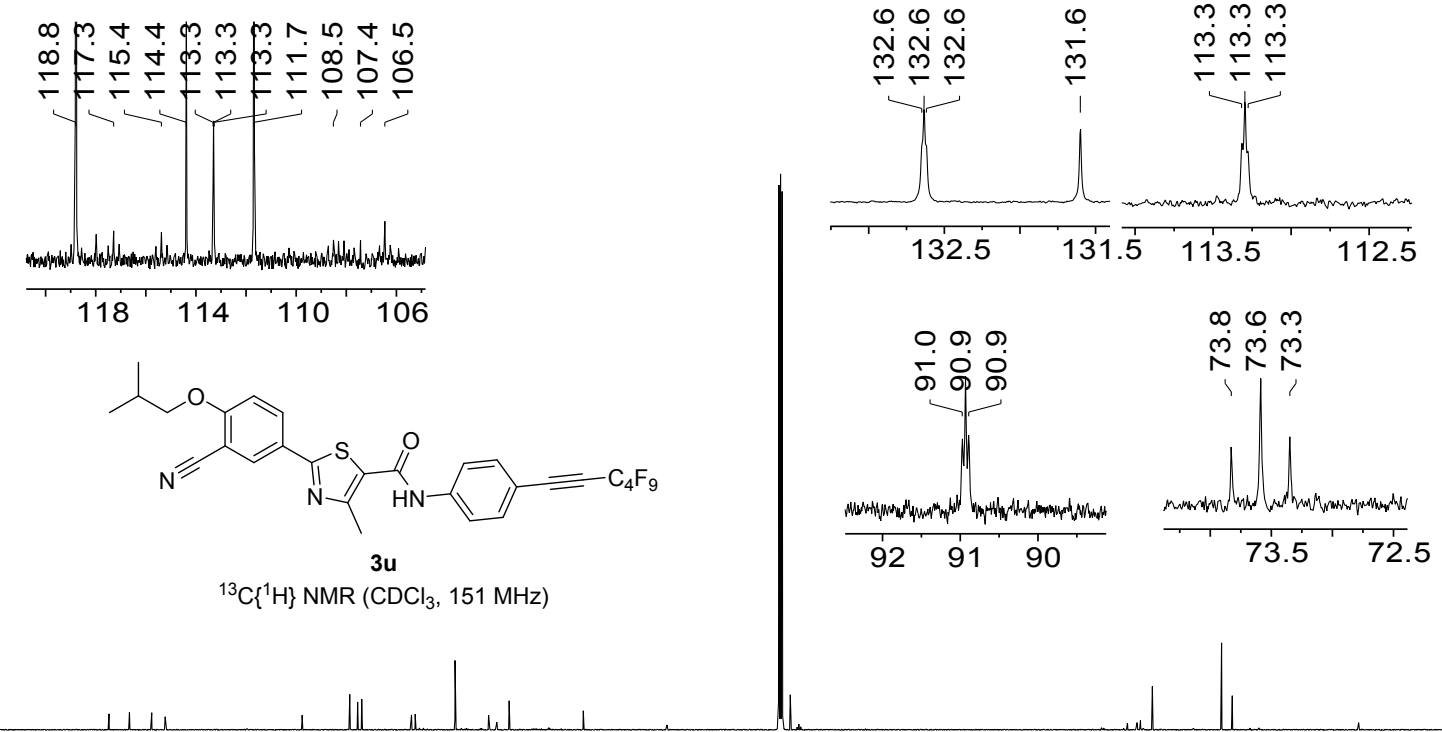

m. m.

${ }^{13} \mathrm{C}\left\{{ }^{1} \mathrm{H}\right\} \mathrm{NMR}\left(\mathrm{CDCl}_{3}, 151 \mathrm{MHz}\right)$

$\begin{array}{lllllllllllllllllll}170 & 160 & 150 & 140 & 130 & 120 & 110 & 100 & 90 & 80 & 70 & 60 & 50 & 40 & 30 & 20 & 10 & 0 & -1\end{array}$ 


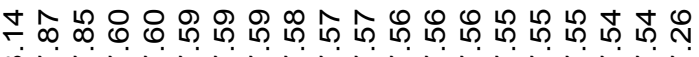

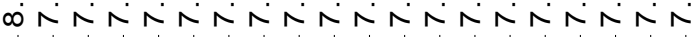

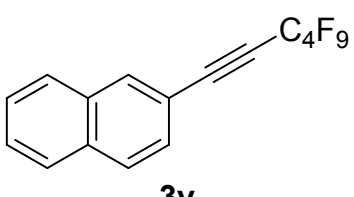

3v

${ }^{1} \mathrm{H} \mathrm{NMR}\left(\mathrm{CDCl}_{3}, 600 \mathrm{MHz}\right)$

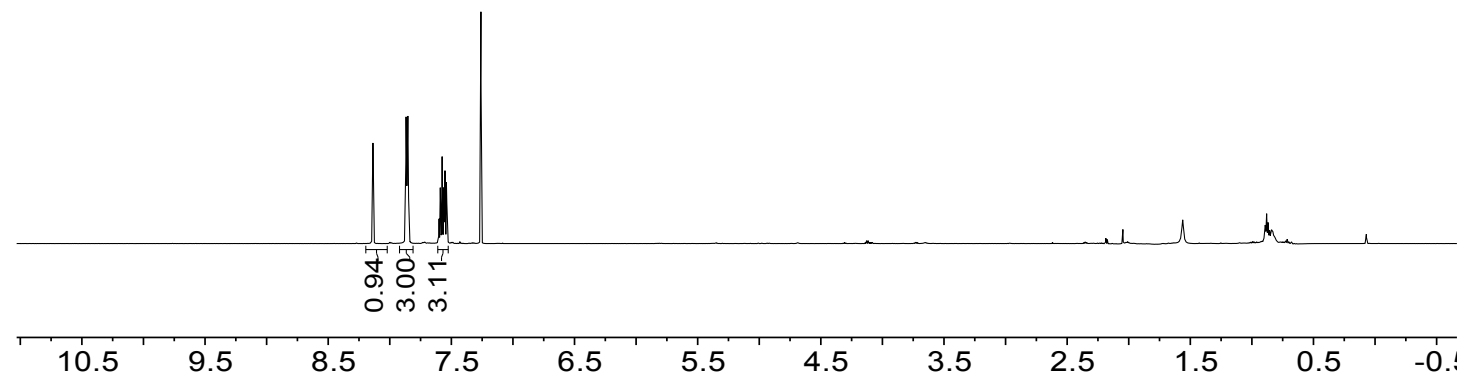

$\begin{array}{lll}9 & 9 & 0 \\ 0 & 0 & 0 \\ \infty & \infty & \infty\end{array}$

ก บ พ บ พ

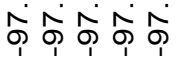

$m m m m m m m+\forall \forall+$

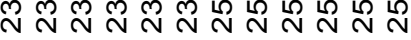

T)

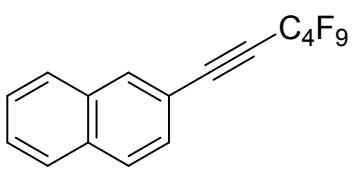

$3 v$

${ }^{19} \mathrm{~F} \mathrm{NMR}\left(\mathrm{CDCl}_{3}, 565 \mathrm{MHz}\right)$

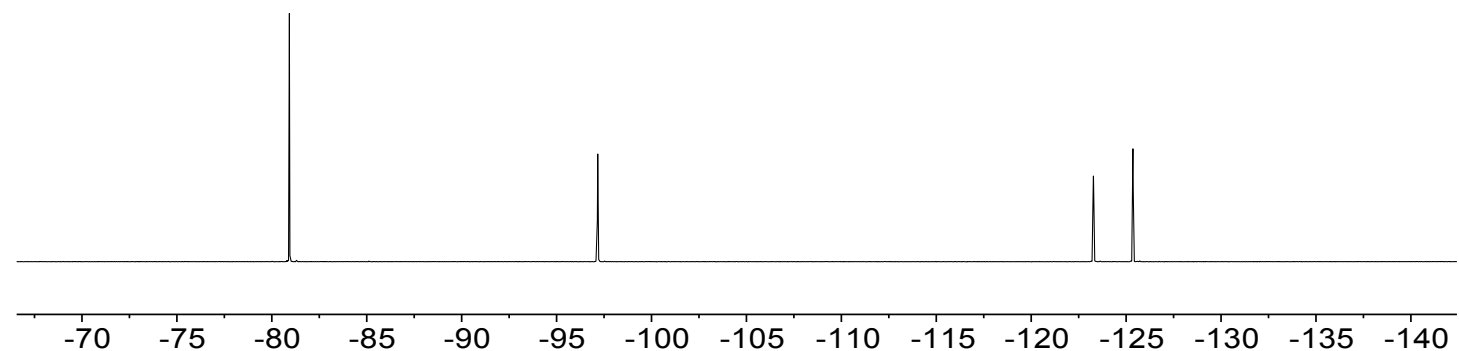




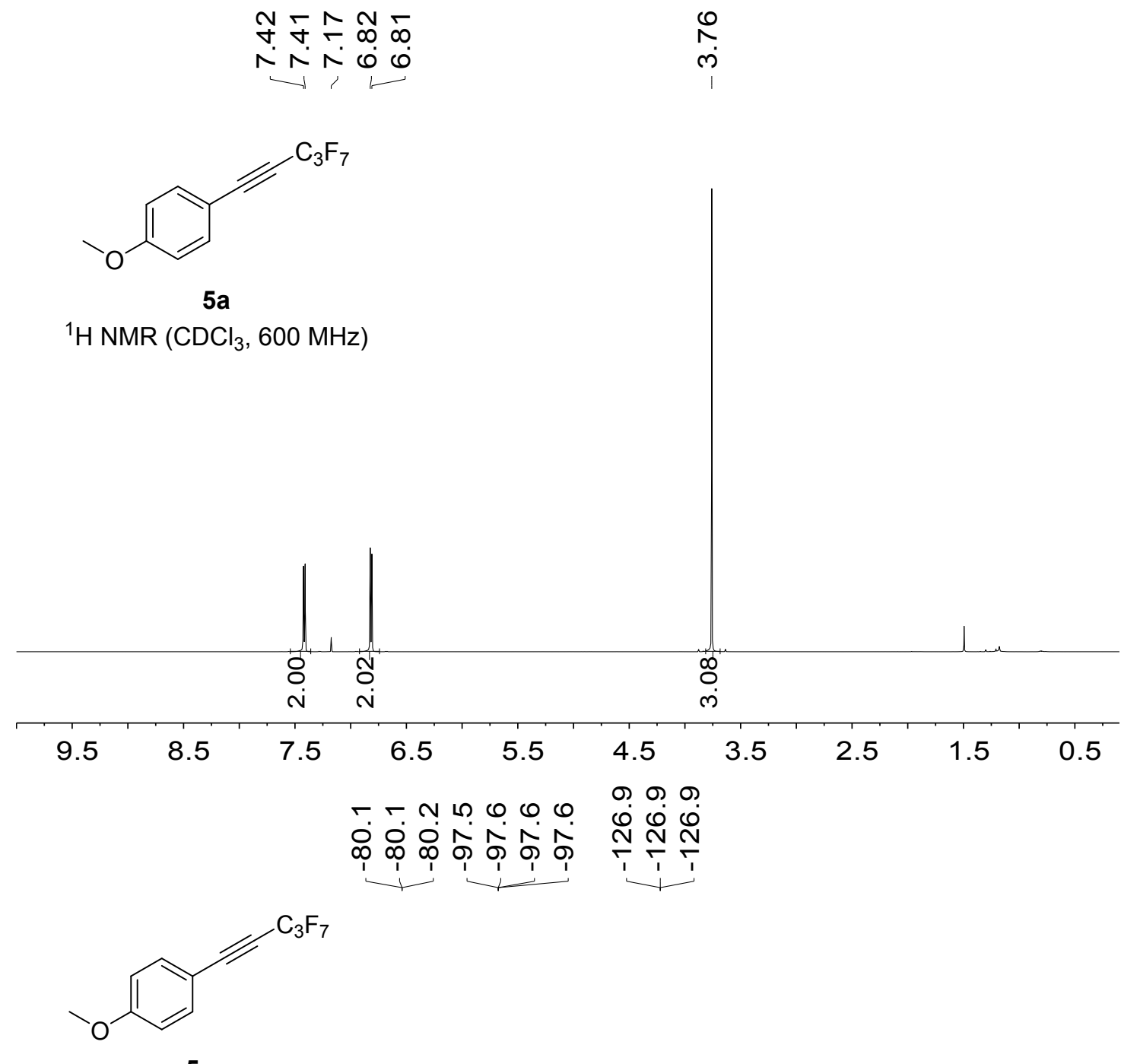

$5 a$

${ }^{19} \mathrm{~F} \mathrm{NMR}\left(\mathrm{CDCl}_{3}, 565 \mathrm{MHz}\right)$

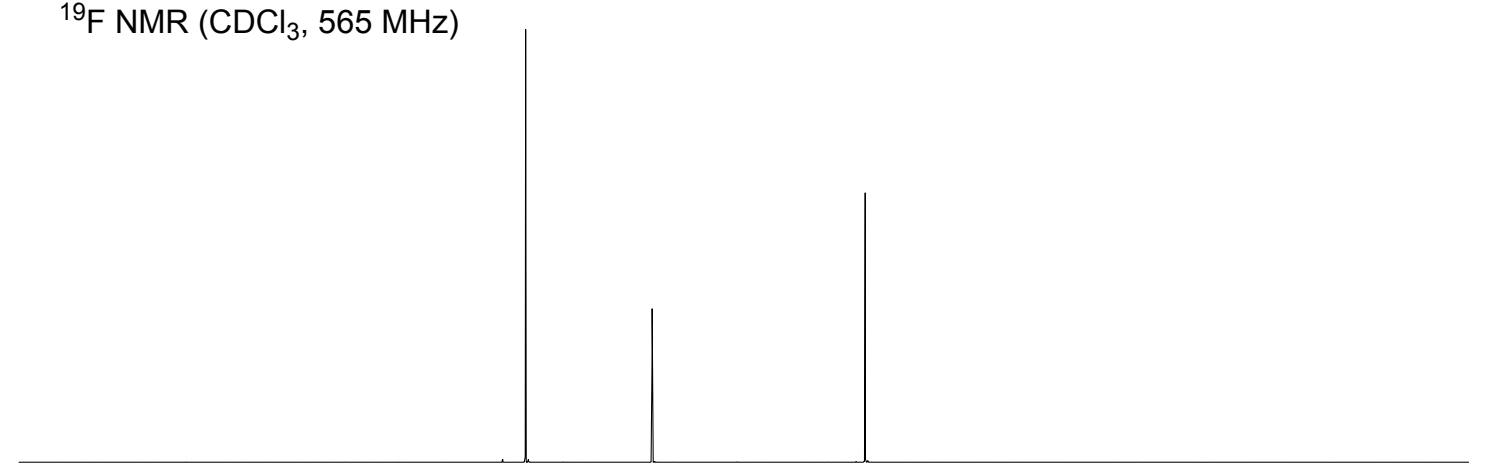

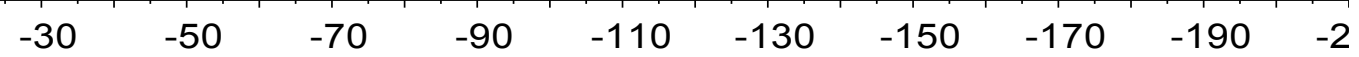




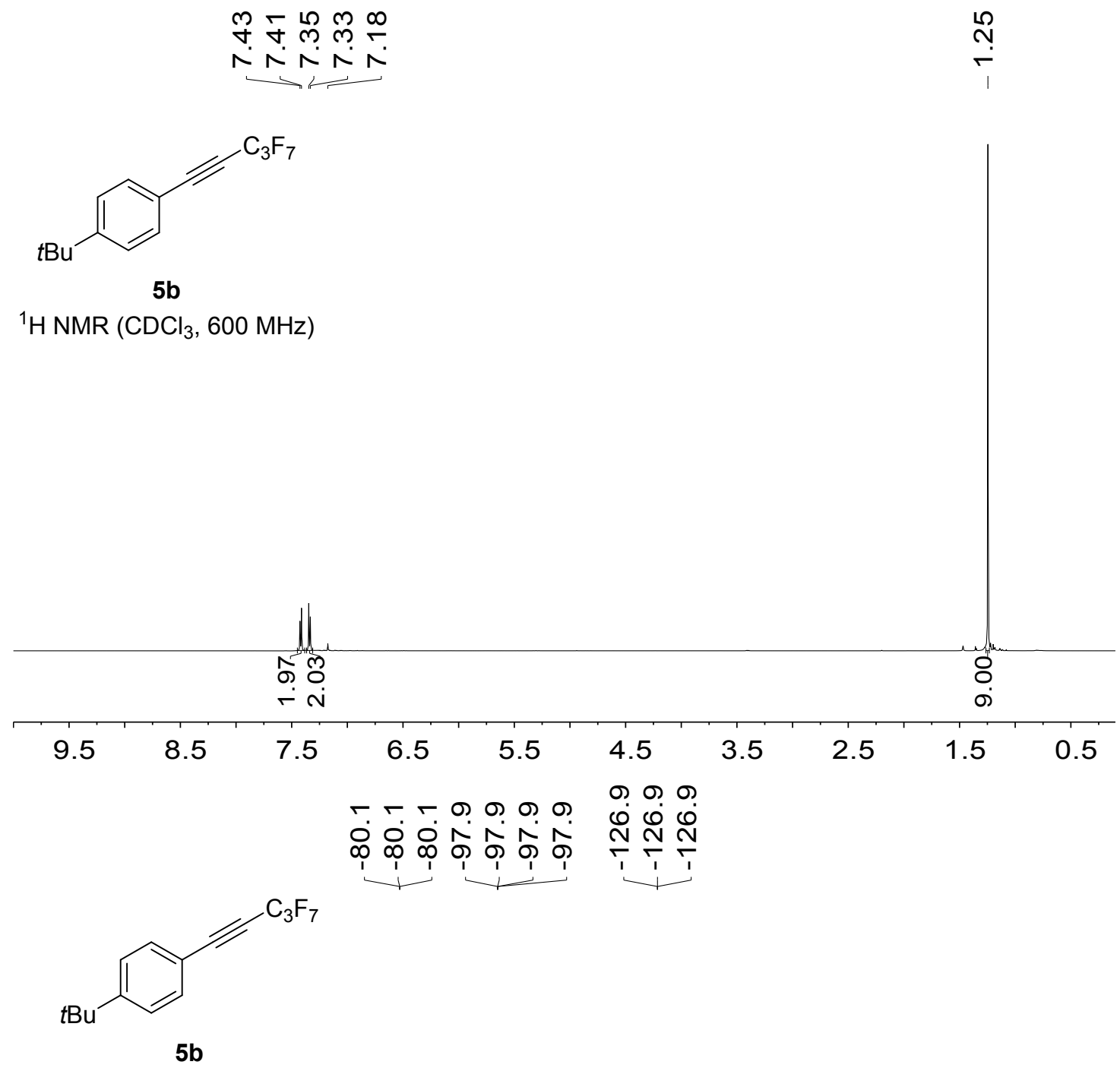

${ }^{19} \mathrm{~F} \mathrm{NMR}\left(\mathrm{CDCl}_{3}, 565 \mathrm{MHz}\right)$

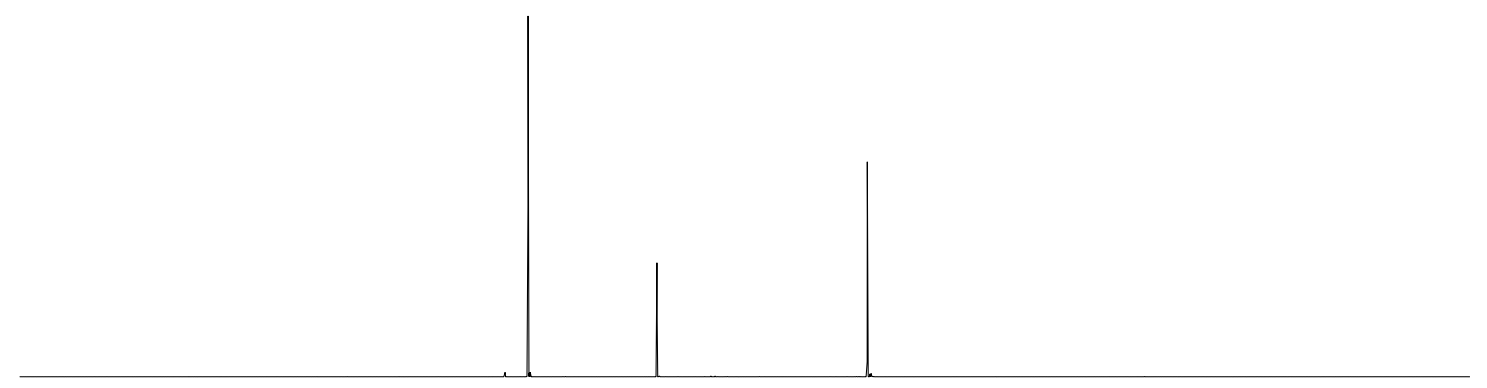

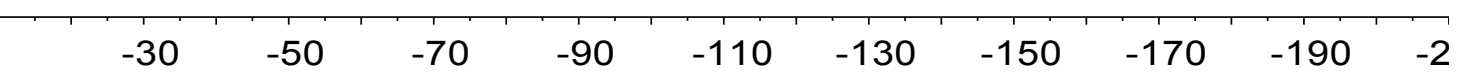


$\stackrel{m}{\forall} \underset{\sim}{\sim} \stackrel{\infty}{\leftarrow} \underset{\infty}{\infty} \infty$

$\sim \wedge$ ○

$\stackrel{0}{N}$

+1,

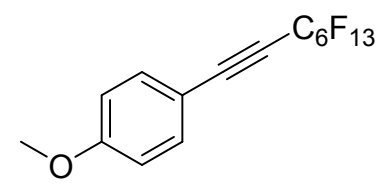

$6 a$

${ }^{1} \mathrm{H} \mathrm{NMR}\left(\mathrm{CDCl}_{3}, 600 \mathrm{MHz}\right)$

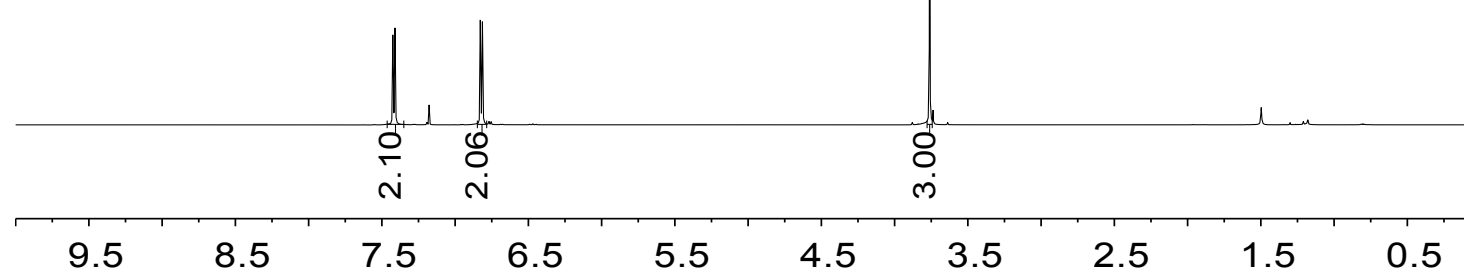

のののの の ๓

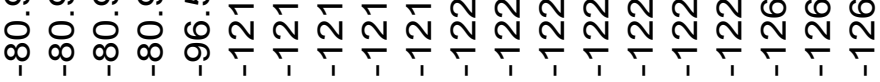

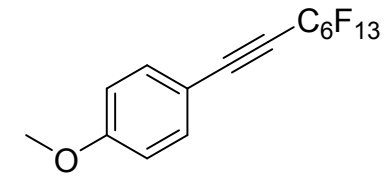

$6 a$

${ }^{19} \mathrm{~F} \mathrm{NMR}\left(\mathrm{CDCl}_{3}, 565 \mathrm{MHz}\right)$

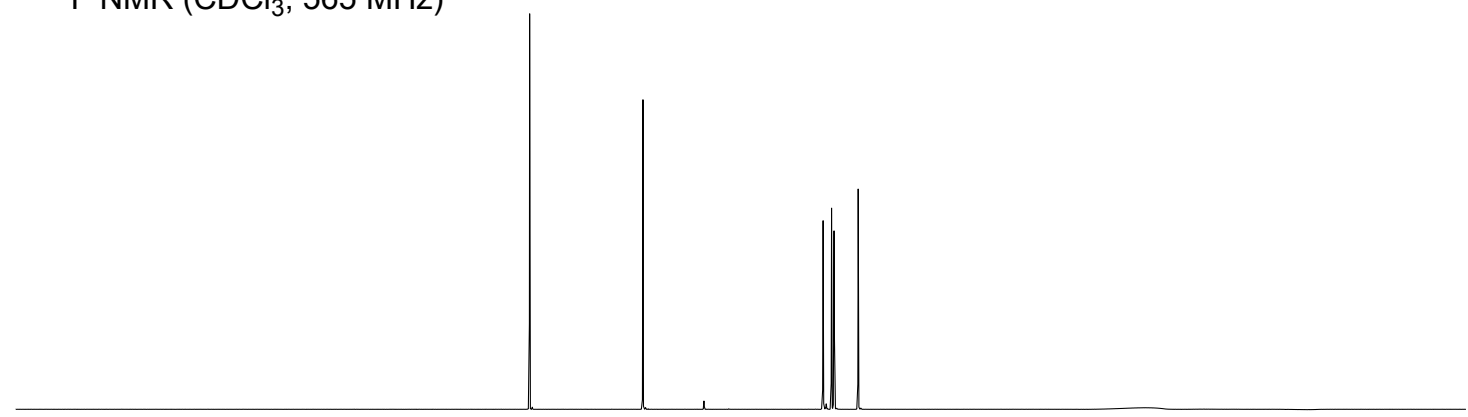

$-30$

$-50$

$-70 \quad-90$

$-110$

$-130$

$-150$

$-170$

$-190 \quad-2$ 


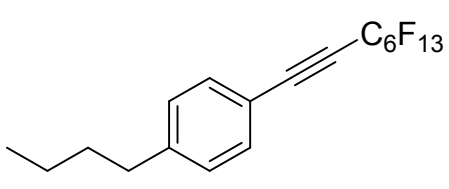

6b

${ }^{1} \mathrm{H} \mathrm{NMR}\left(\mathrm{CDCl}_{3}, 600 \mathrm{MHz}\right)$

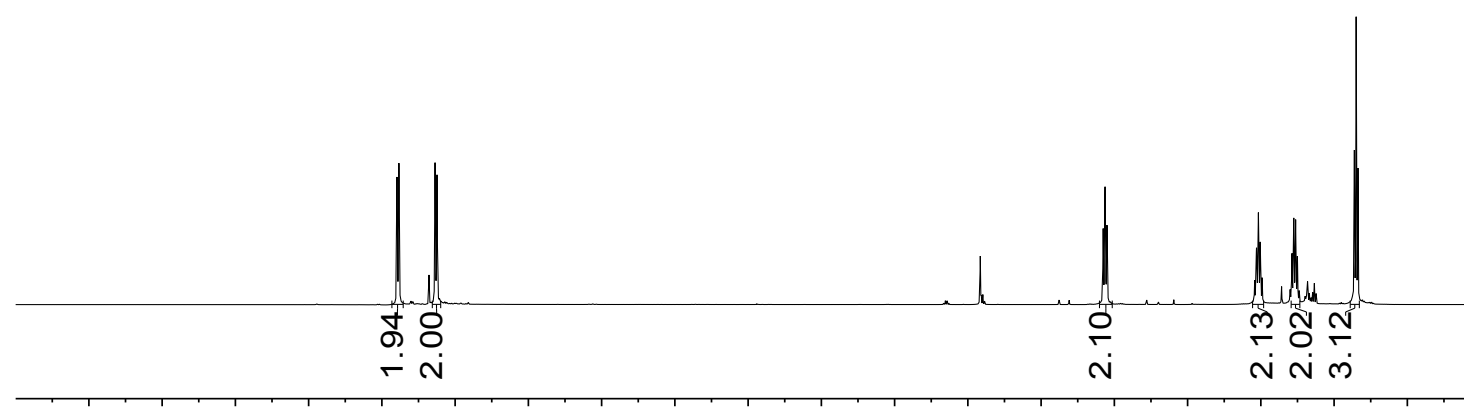

$9.59 .08 .58 .07 .5 \quad 7.06 .56 .05 .5 \quad 5.04 .54 .03 .53 .02 .52 .01 .51 .0 \quad 0.5$

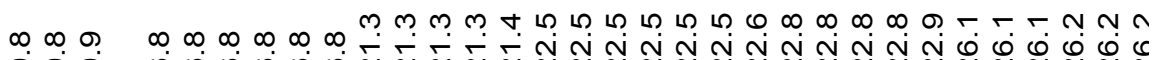

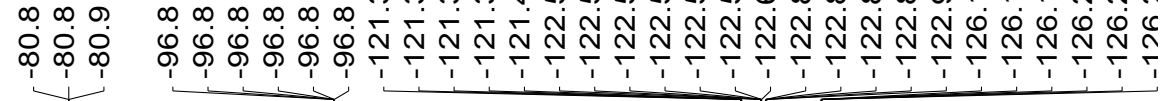

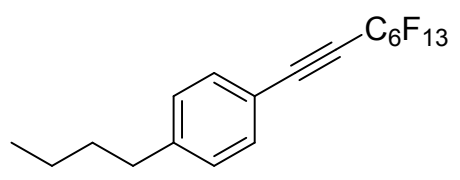

6b

${ }^{19} \mathrm{~F} \mathrm{NMR}\left(\mathrm{CDCl}_{3}, 565 \mathrm{MHz}\right)$

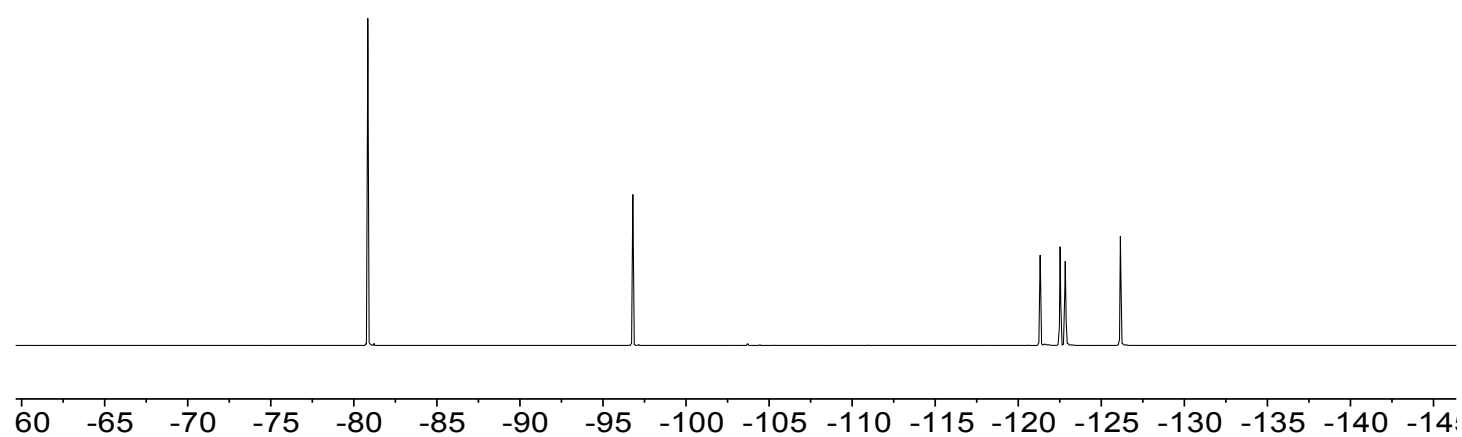


人

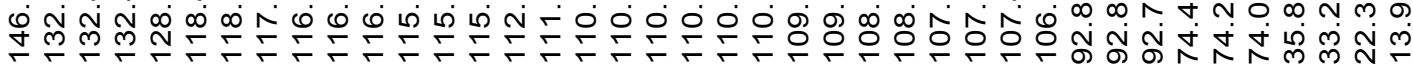

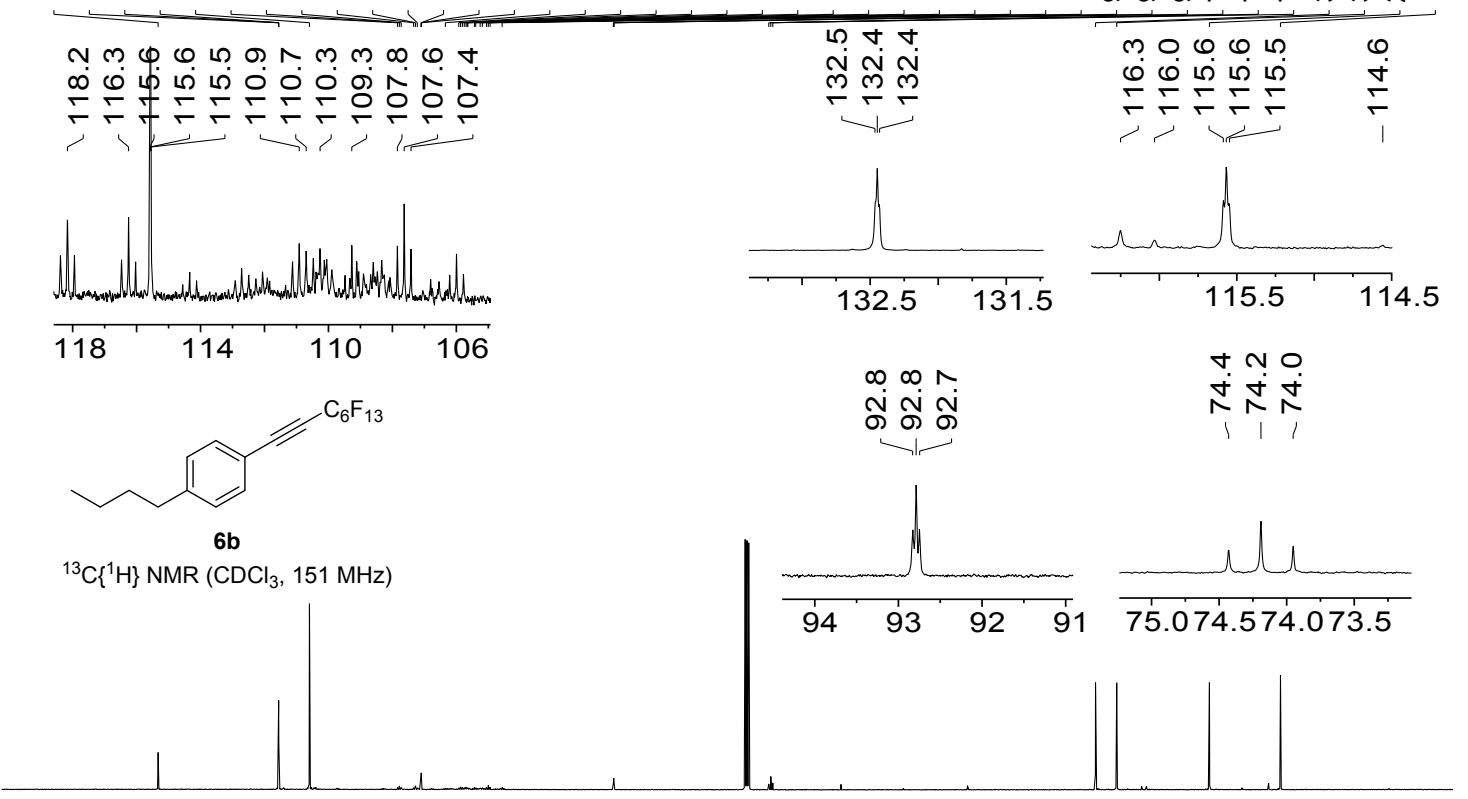

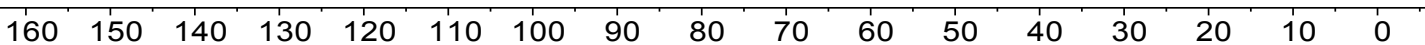

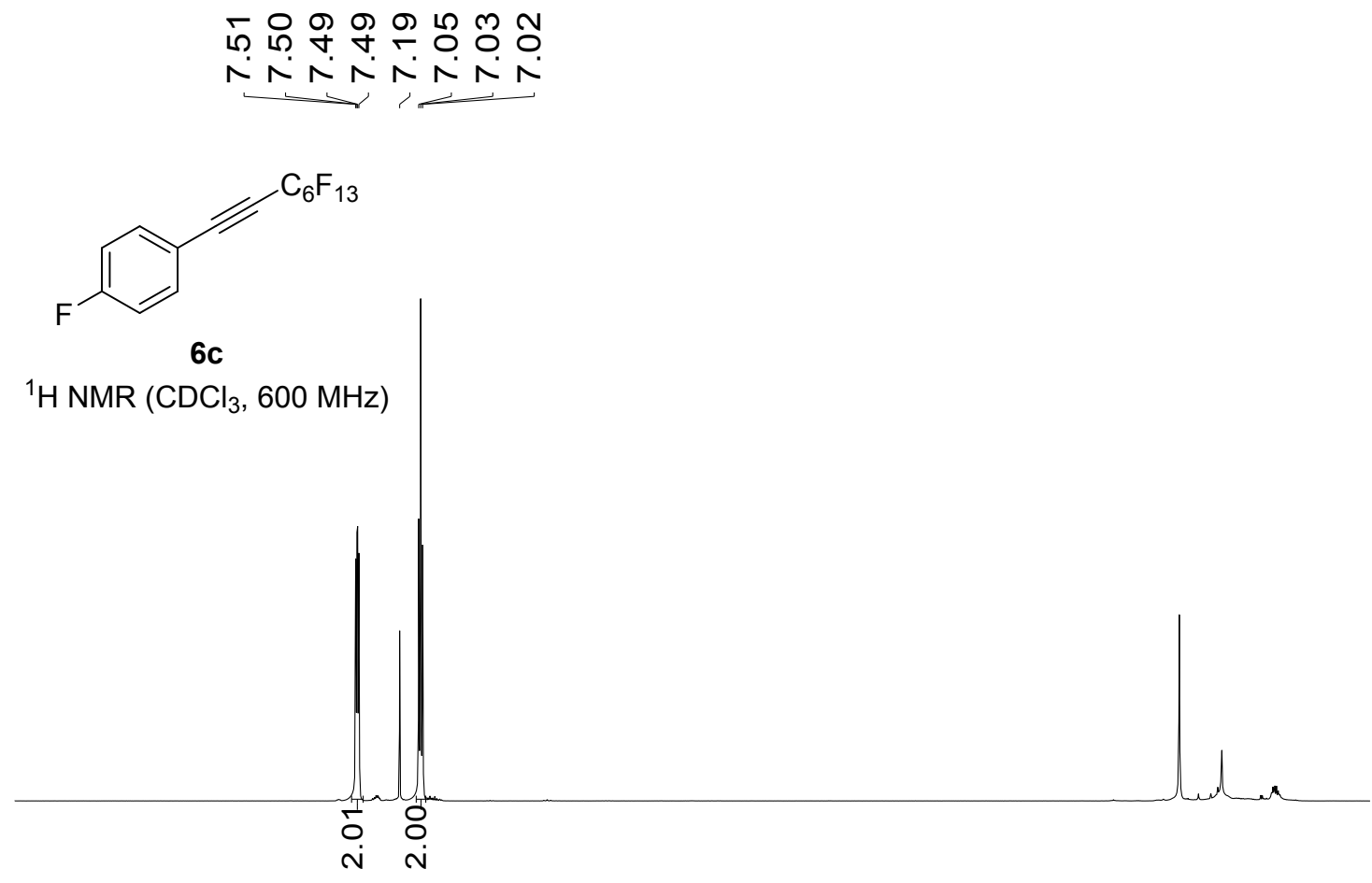

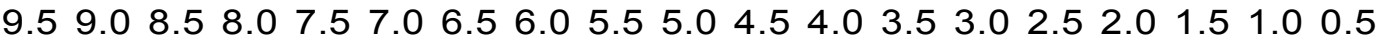




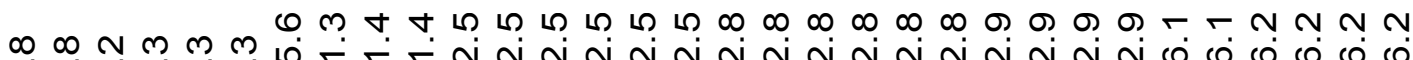
O

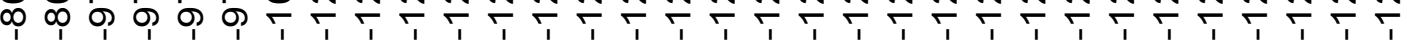

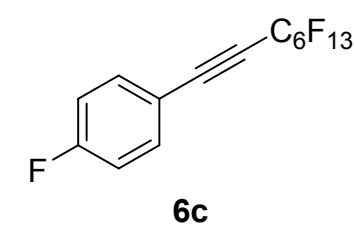

${ }^{19} \mathrm{~F}$ NMR $\left(\mathrm{CDCl}_{3}, 565 \mathrm{MHz}\right)$

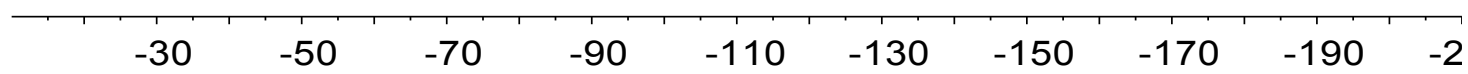

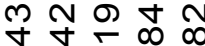

N

$N$,

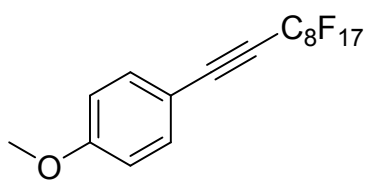

$7 a$

${ }^{1} \mathrm{H} \mathrm{NMR}\left(\mathrm{CDCl}_{3}, 600 \mathrm{MHz}\right)$

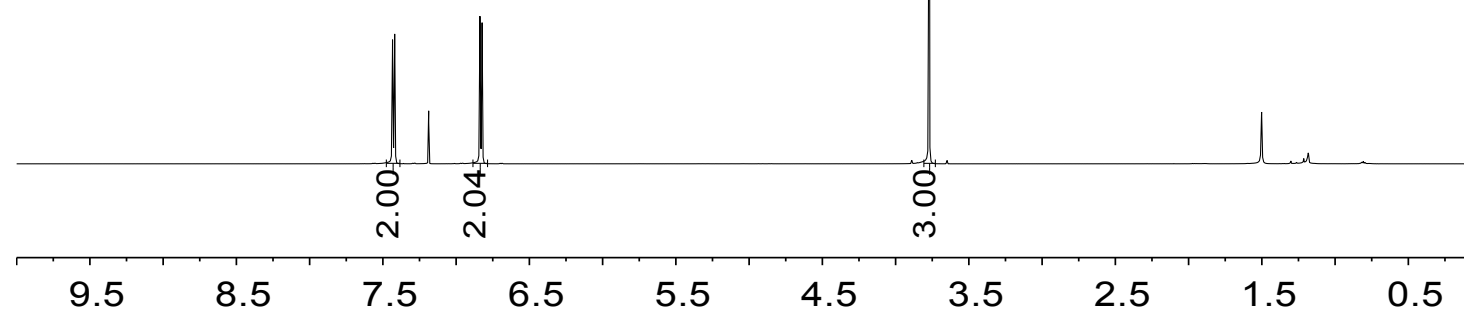


$\infty$ 我

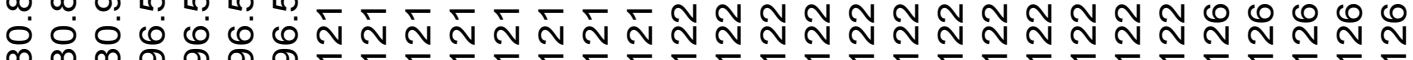

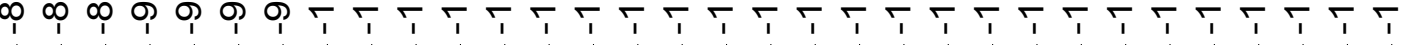

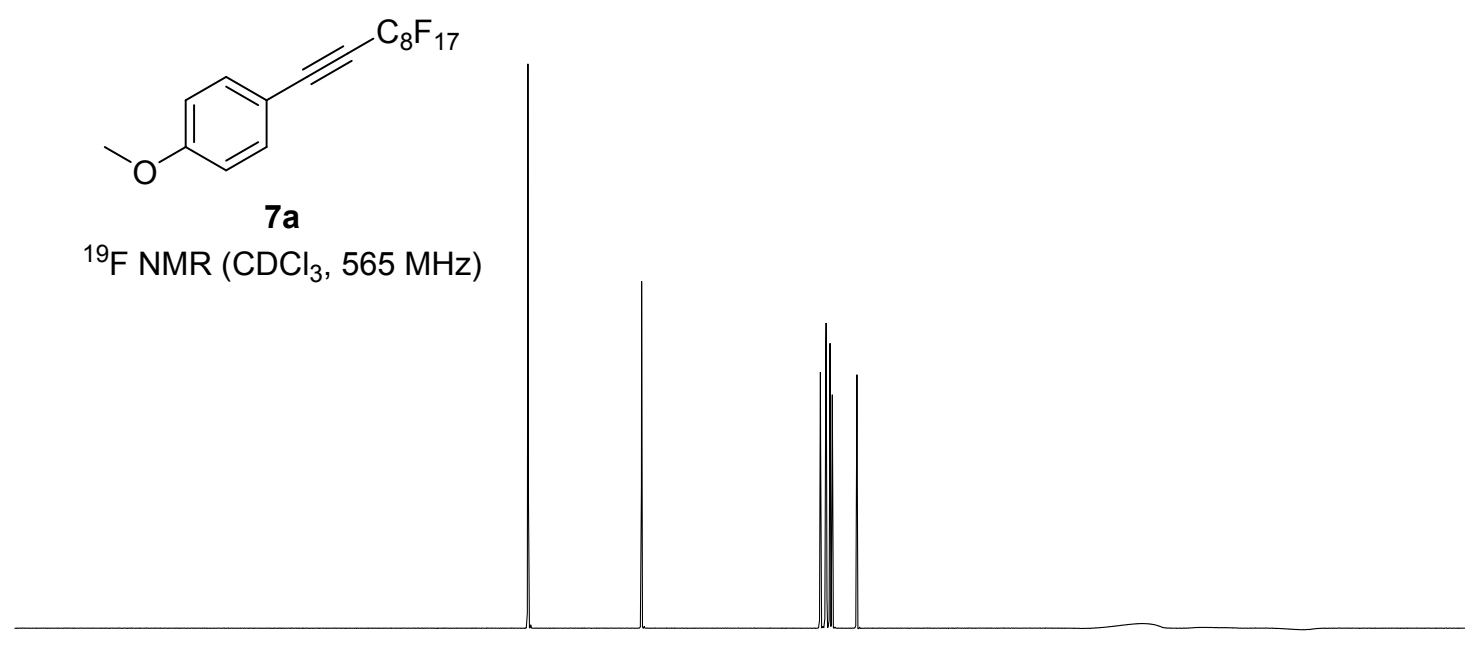

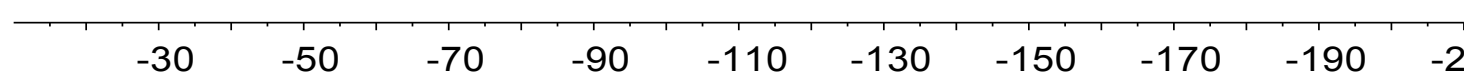

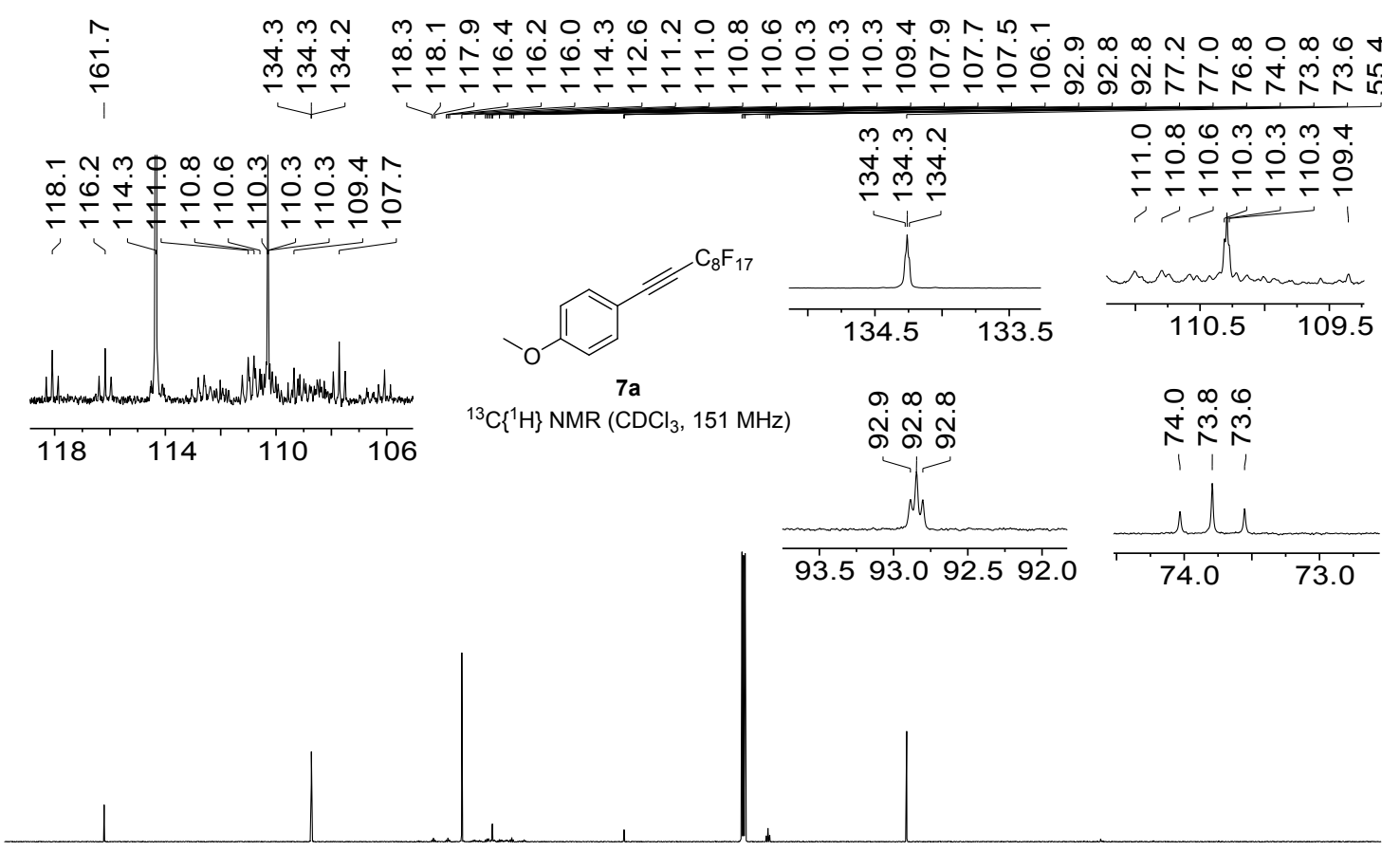

$\begin{array}{llllllllllllllllll}170 & 160 & 150 & 140 & 130 & 120 & 110 & 100 & 90 & 80 & 70 & 60 & 50 & 40 & 30 & 20 & 10 & 0\end{array}$ 


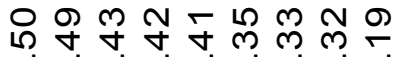

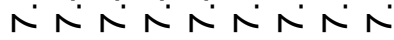

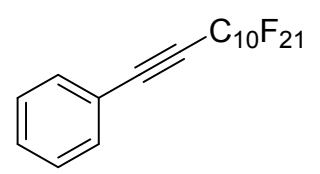

$8 \mathbf{a}$

${ }^{1} \mathrm{H} \mathrm{NMR}\left(\mathrm{CDCl}_{3}, 600 \mathrm{MHz}\right)$

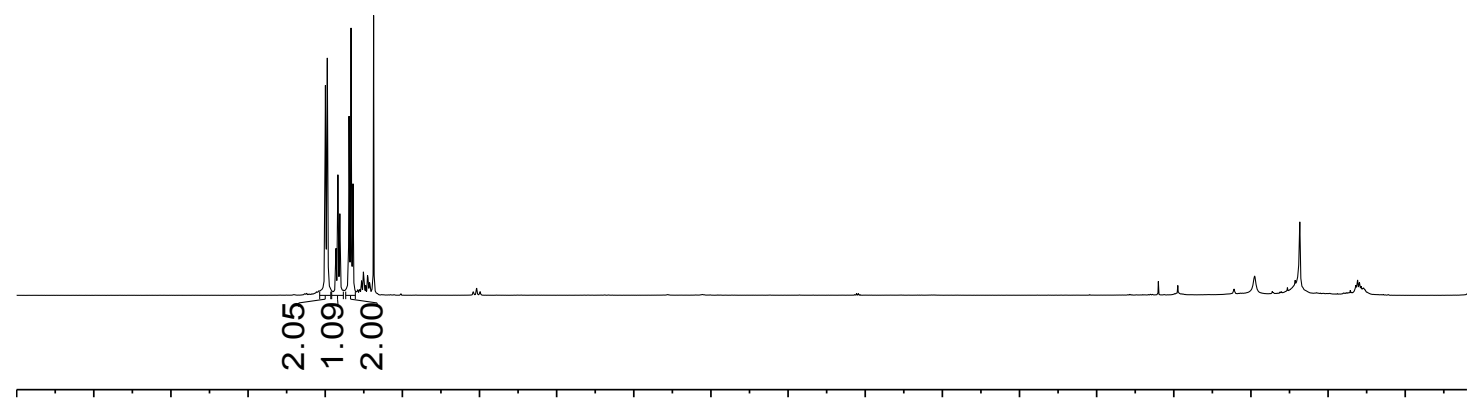

$\begin{array}{lllllllllllllllllll}.5 & 9.0 & 8.5 & 8.0 & 7.5 & 7.0 & 6.5 & 6.0 & 5.5 & 5.0 & 4.5 & 4.0 & 3.5 & 3.0 & 2.5 & 2.0 & 1.5 & 1.0 & 0.5\end{array}$

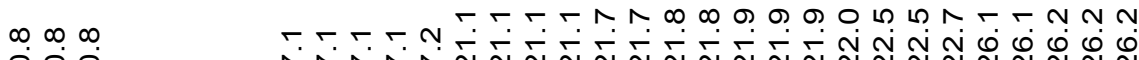

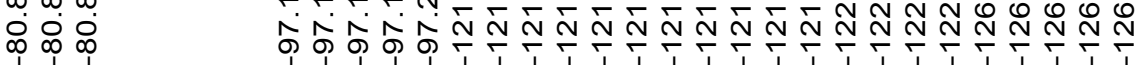

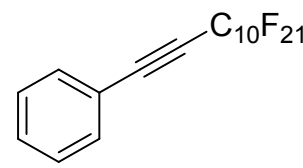

$8 \mathbf{a}$

${ }^{19} \mathrm{~F} \mathrm{NMR}\left(\mathrm{CDCl}_{3}, 565 \mathrm{MHz}\right)$

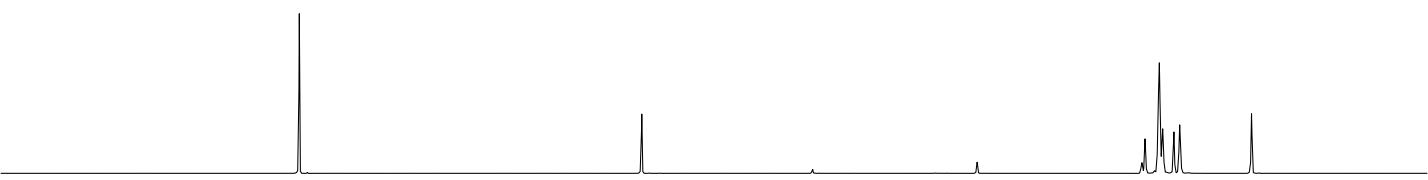

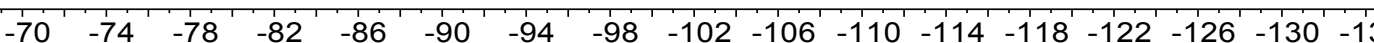




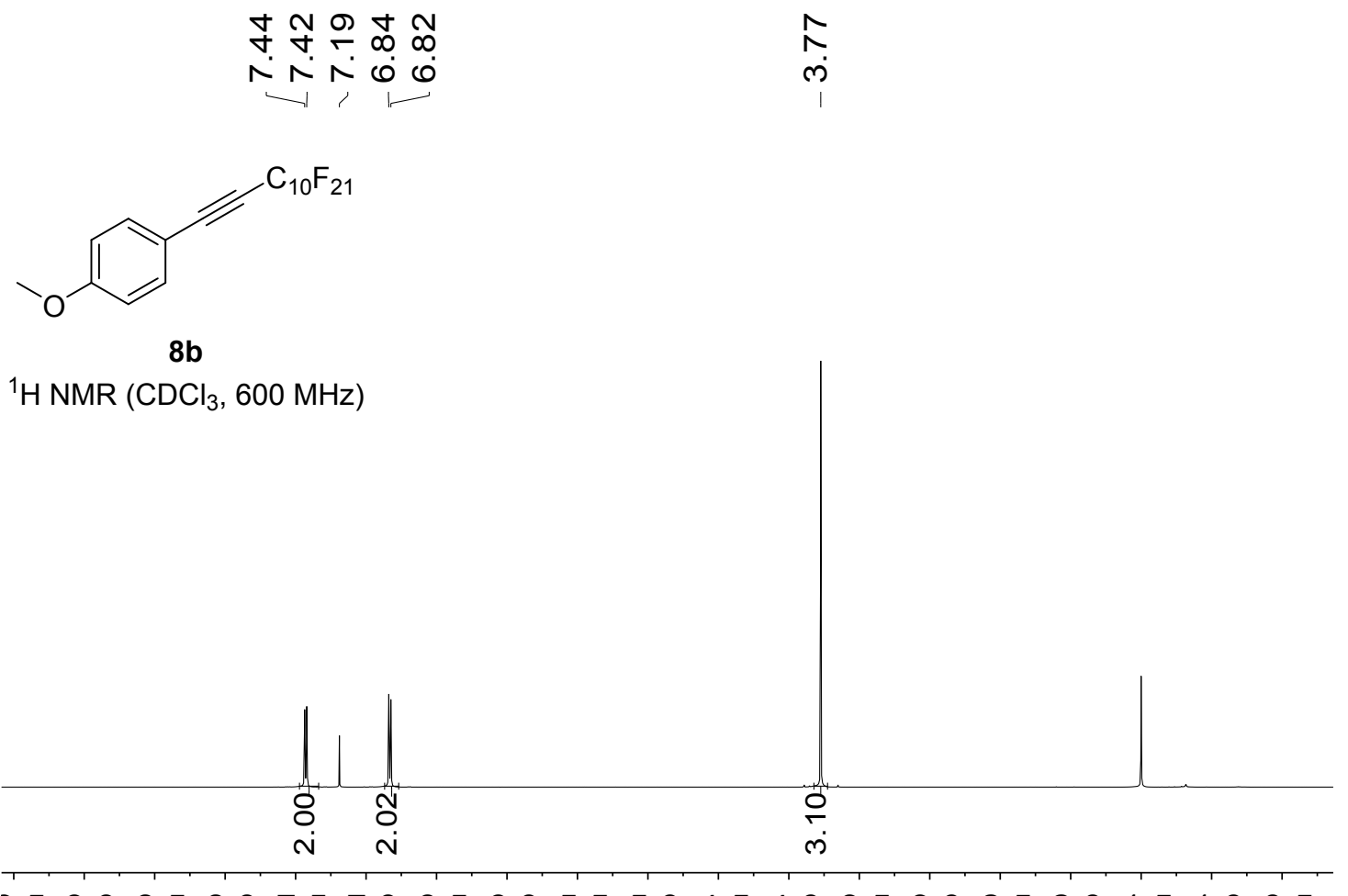

$\begin{array}{lllllllllllllllllll}3.5 & 9.0 & 8.5 & 8.0 & 7.5 & 7.0 & 6.5 & 6.0 & 5.5 & 5.0 & 4.5 & 4.0 & 3.5 & 3.0 & 2.5 & 2.0 & 1.5 & 1.0 & 0.5\end{array}$

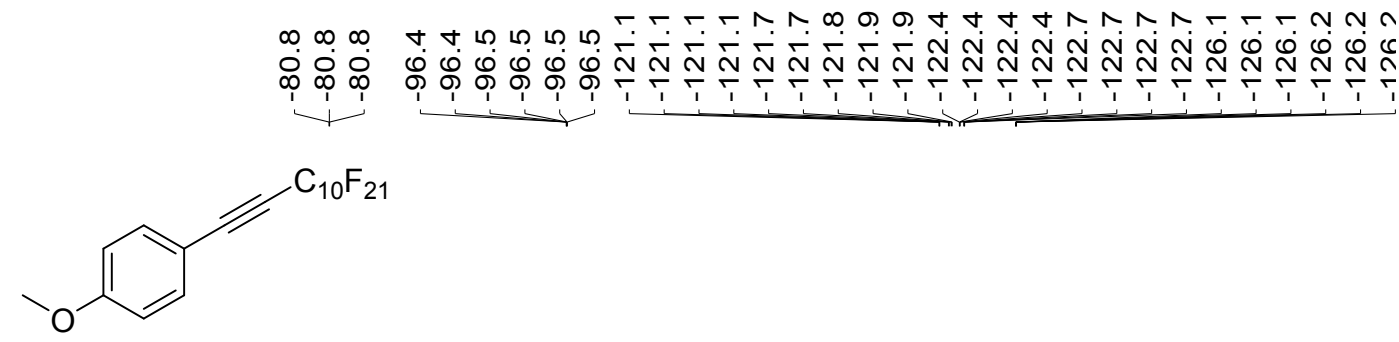

$8 b$

${ }^{19} \mathrm{~F} \mathrm{NMR}\left(\mathrm{CDCl}_{3}, 565 \mathrm{MHz}\right)$

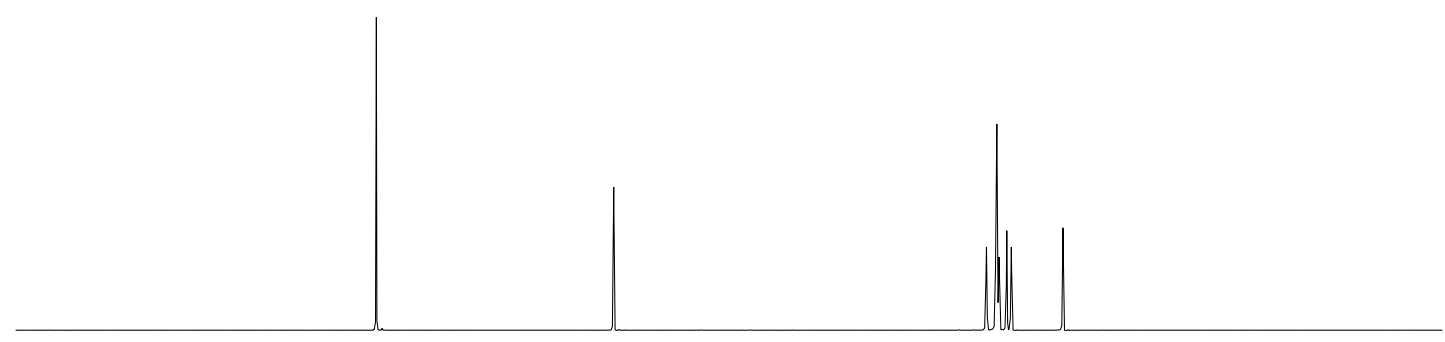

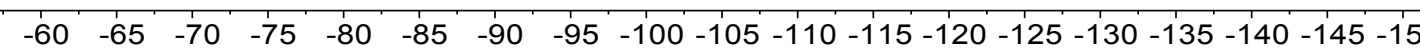




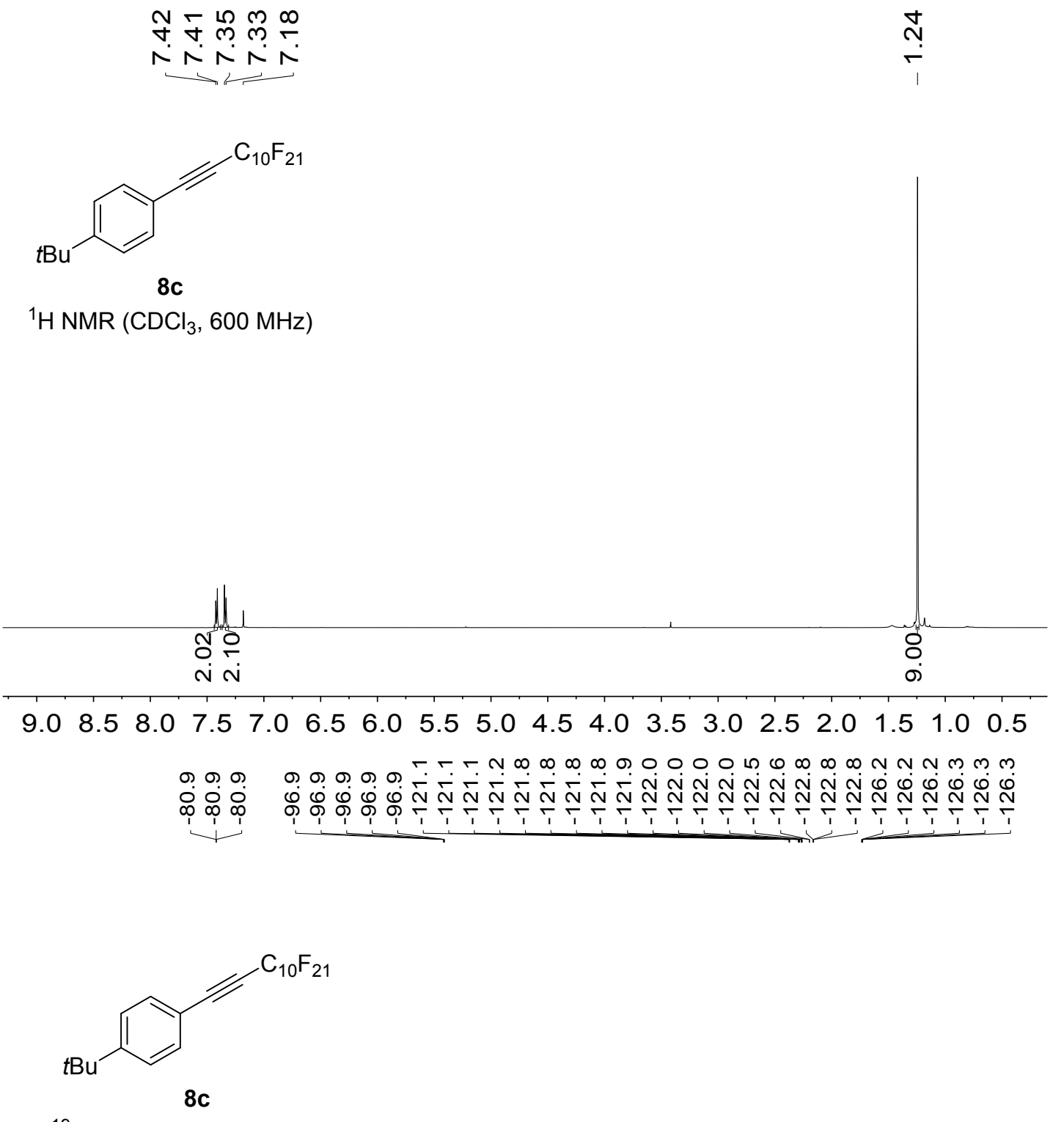

${ }^{19} \mathrm{~F} \mathrm{NMR}\left(\mathrm{CDCl}_{3}, 565 \mathrm{MHz}\right)$

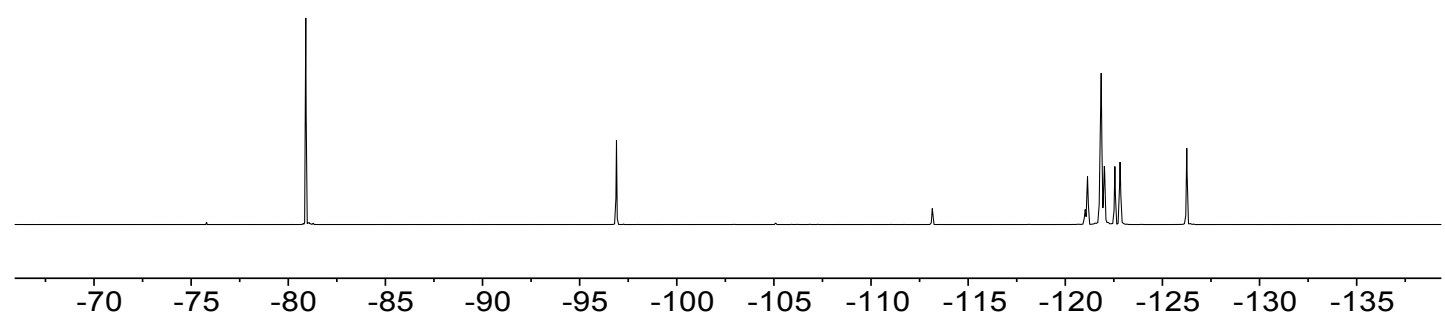


$\infty m m$ m $m$ m $m$ m

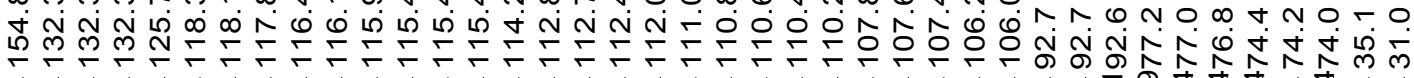

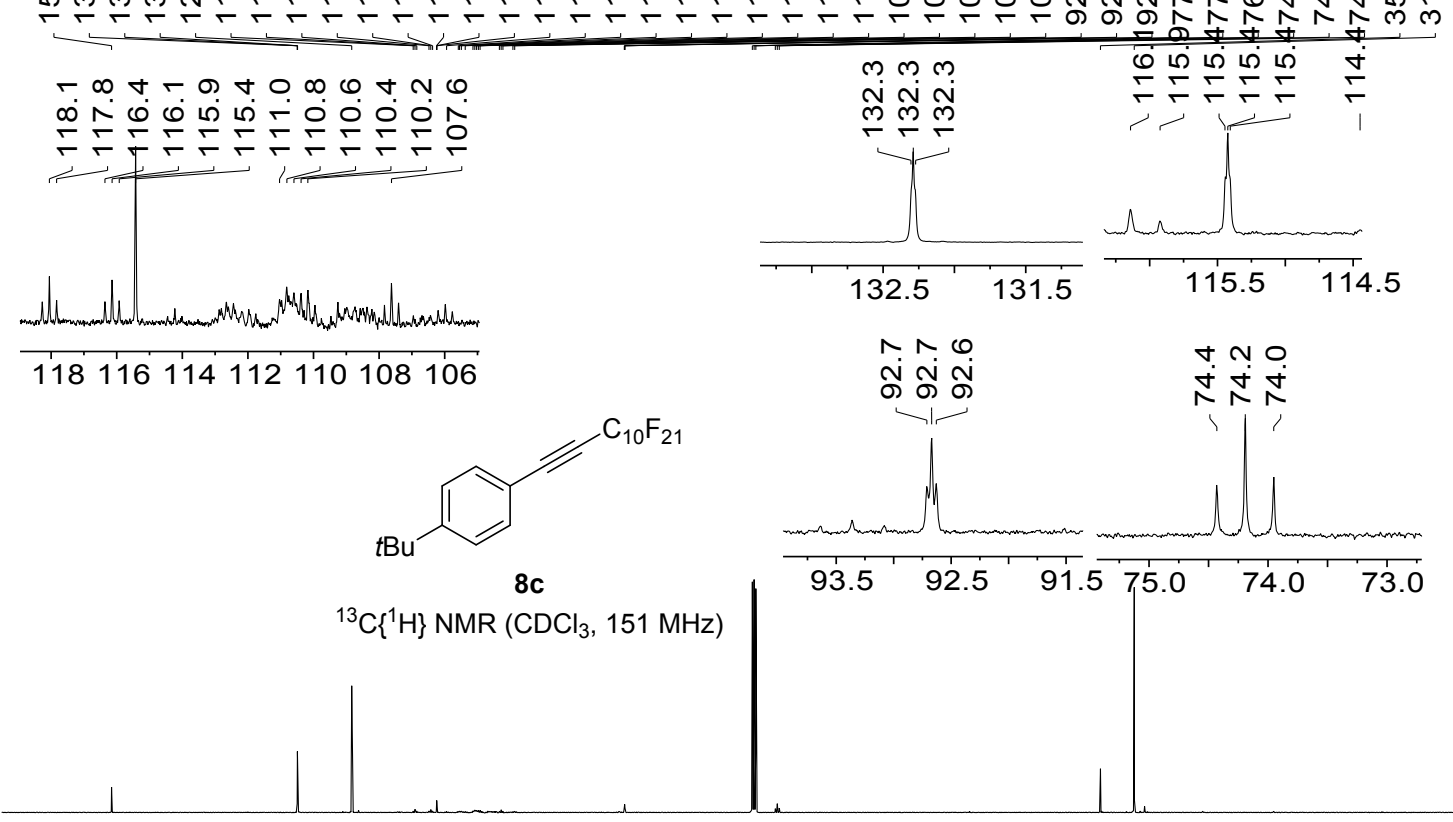

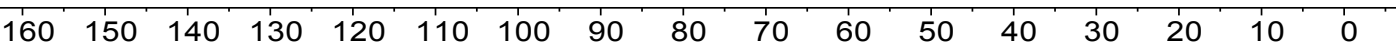

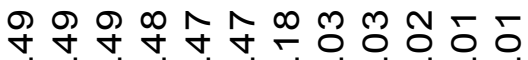

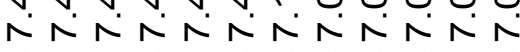

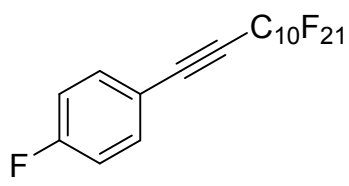

8d

${ }^{1} \mathrm{H} \mathrm{NMR}\left(\mathrm{CDCl}_{3}, 600 \mathrm{MHz}\right)$

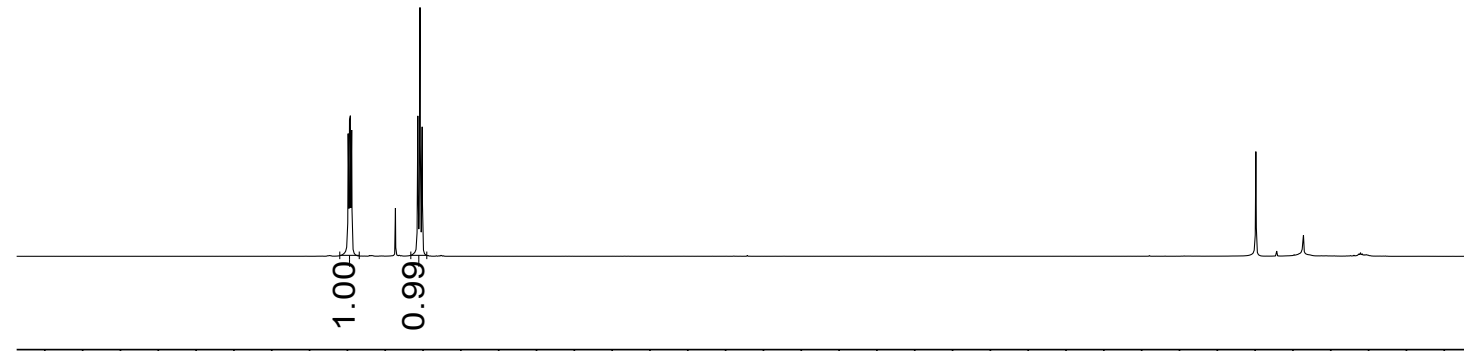

$\begin{array}{lllllllllllllllllll}9.5 & 9.0 & 8.5 & 8.0 & 7.5 & 7.0 & 6.5 & 6.0 & 5.5 & 5.0 & 4.5 & 4.0 & 3.5 & 3.0 & 2.5 & 2.0 & 1.5 & 1.0 & 0.5\end{array}$ 


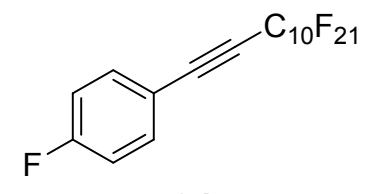

8d

${ }^{19} \mathrm{~F} \mathrm{NMR}\left(\mathrm{CDCl}_{3}, 565 \mathrm{MHz}\right)$

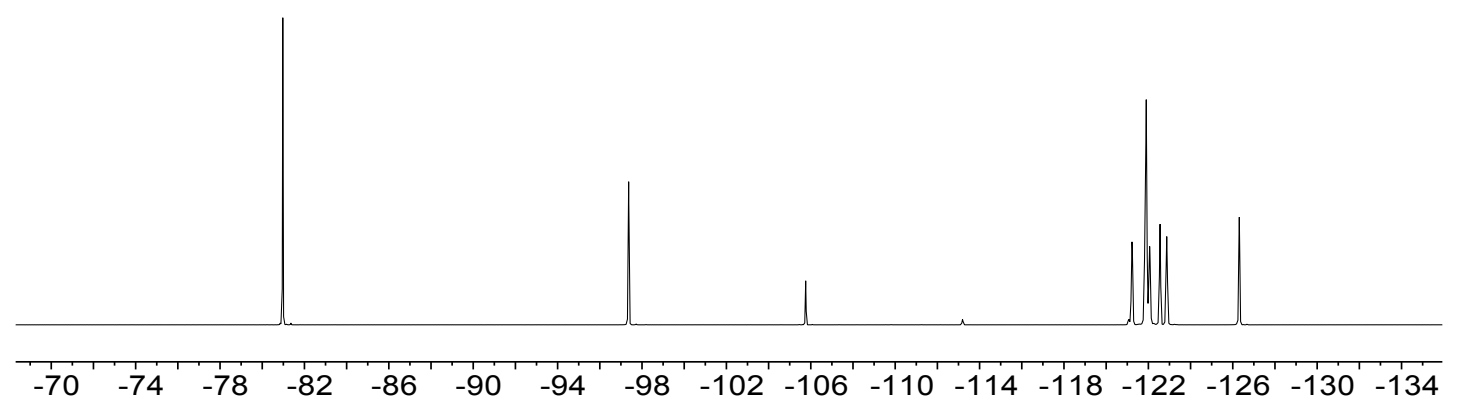

$\stackrel{\oplus}{\oplus}$

ז<smiles>[PH2+]CC#CC1CC1</smiles>

$8 e$

${ }^{1} \mathrm{H} \mathrm{NMR}\left(\mathrm{CDCl}_{3}, 600 \mathrm{MHz}\right)$

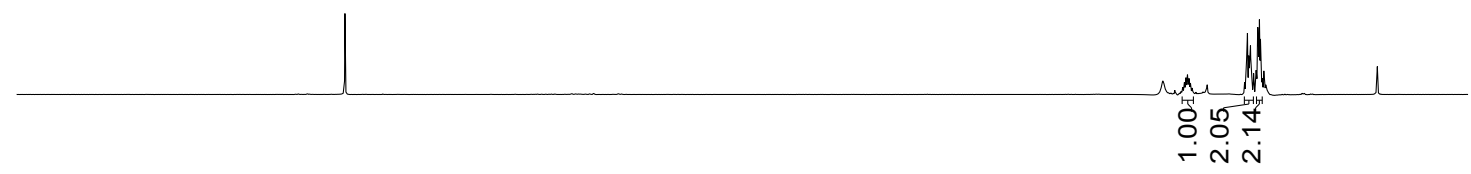

\begin{tabular}{lllllllllllllllllllllllll}
\hline .5 & 9.0 & 8.5 & 8.0 & 7.5 & 7.0 & 6.5 & 6.0 & 5.5 & 5.0 & 4.5 & 4.0 & 3.5 & 3.0 & 2.5 & 2.0 & 1.5 & 1.0 & 0.5 & 0.0 & -0
\end{tabular} 


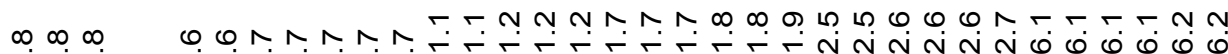

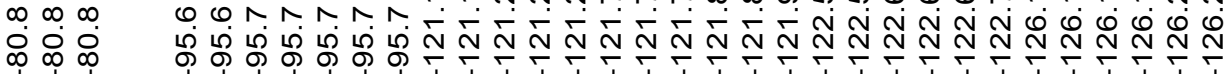

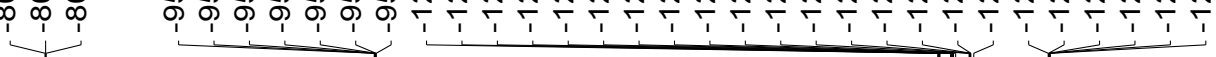<smiles>F[Te]CC#CC1CC1</smiles>

$8 \mathrm{e}$

${ }^{19} \mathrm{~F} \mathrm{NMR}\left(\mathrm{CDCl}_{3}, 565 \mathrm{MHz}\right)$

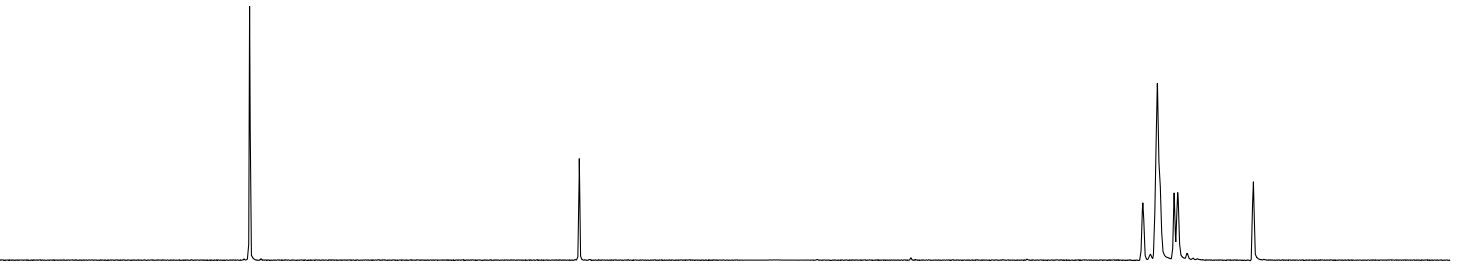

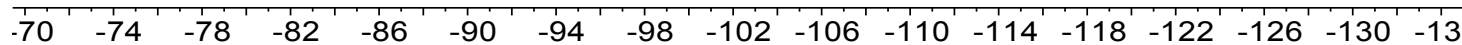

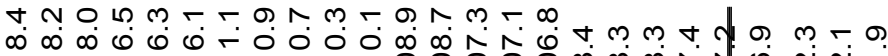

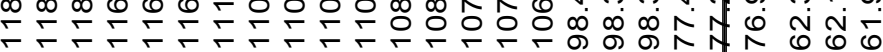

$\circ \infty \infty \infty$

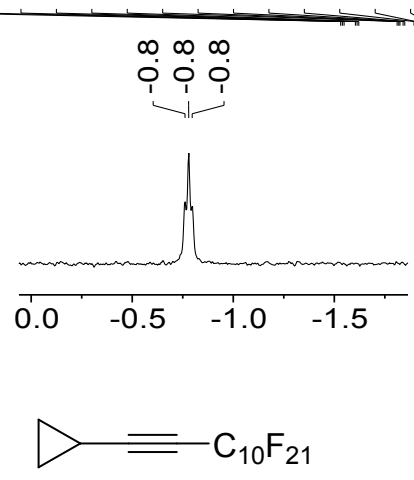

$8 e$

${ }^{13} \mathrm{C}\left\{{ }^{1} \mathrm{H}\right\} \operatorname{NMR}\left(\mathrm{CDCl}_{3}, 151 \mathrm{MHz}\right)$

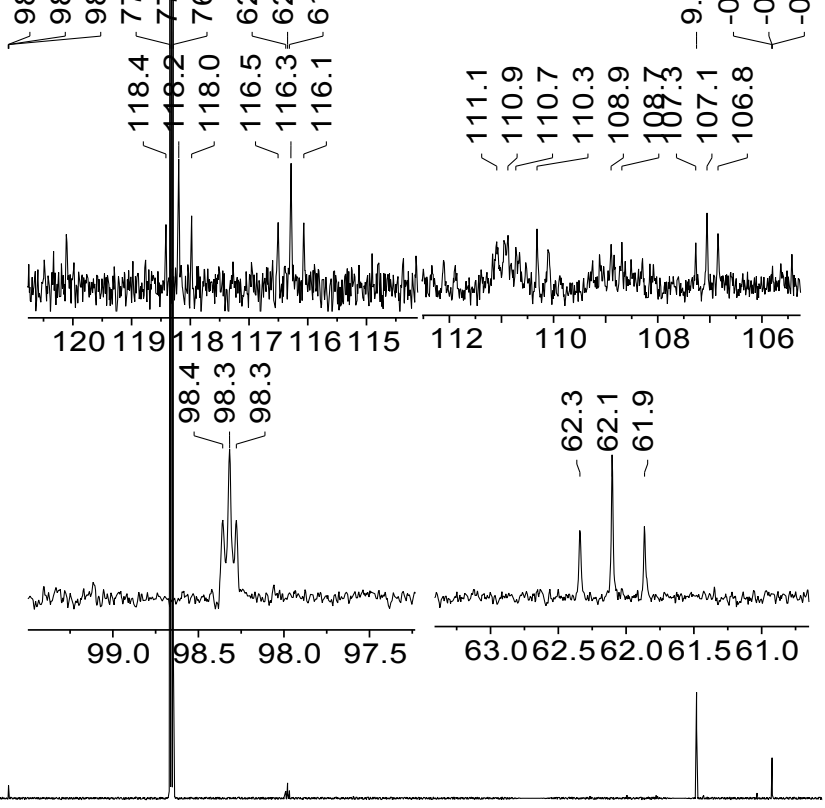

$\begin{array}{lllllllllllllllllll}80 & 170 & 160 & 150 & 140 & 130 & 120 & 110 & 100 & 90 & 80 & 70 & 60 & 50 & 40 & 30 & 20 & 10 & 0\end{array}$ 

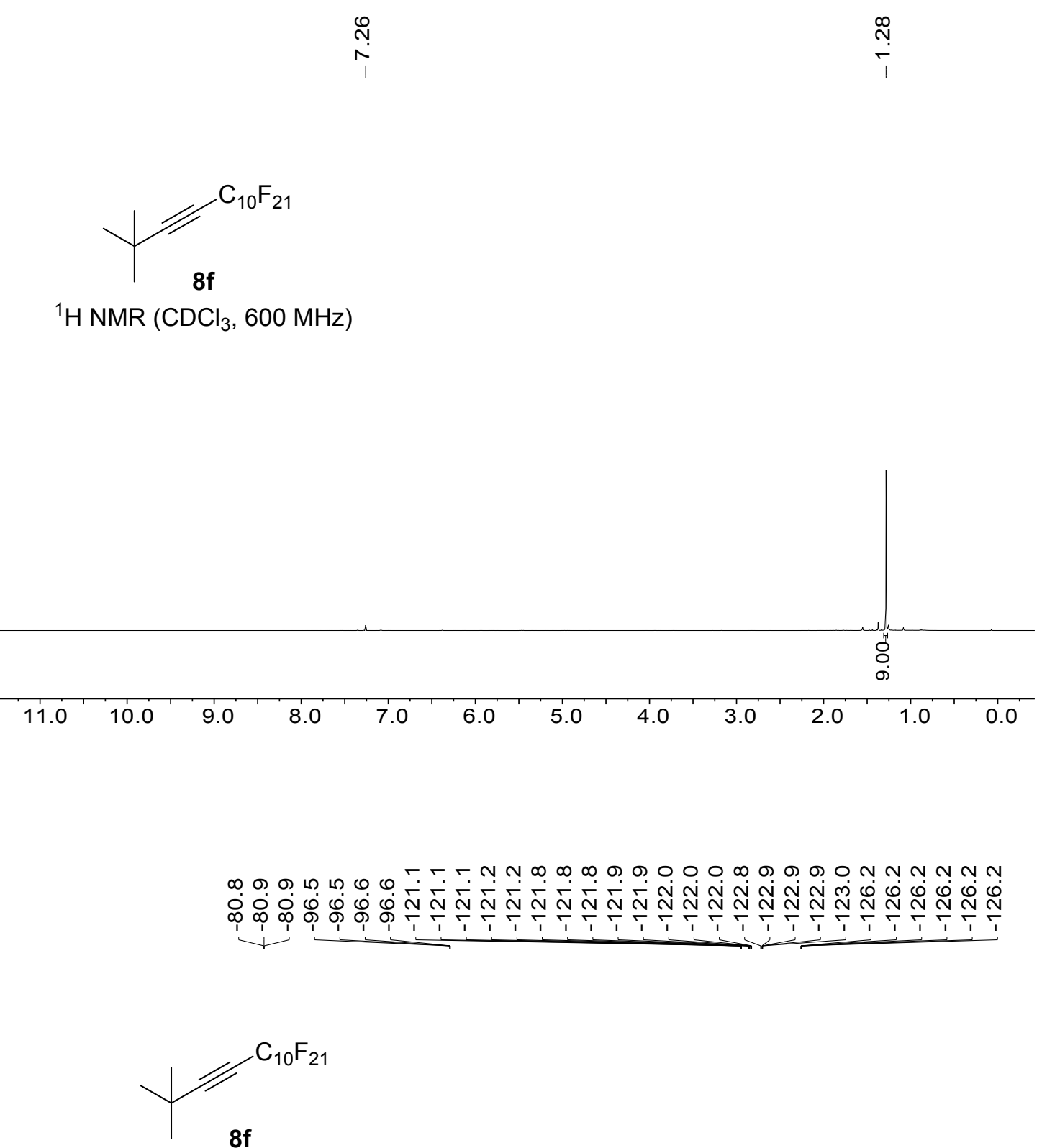

${ }^{19} \mathrm{~F} \mathrm{NMR}\left(\mathrm{CDCl}_{3}, 565 \mathrm{MHz}\right)$

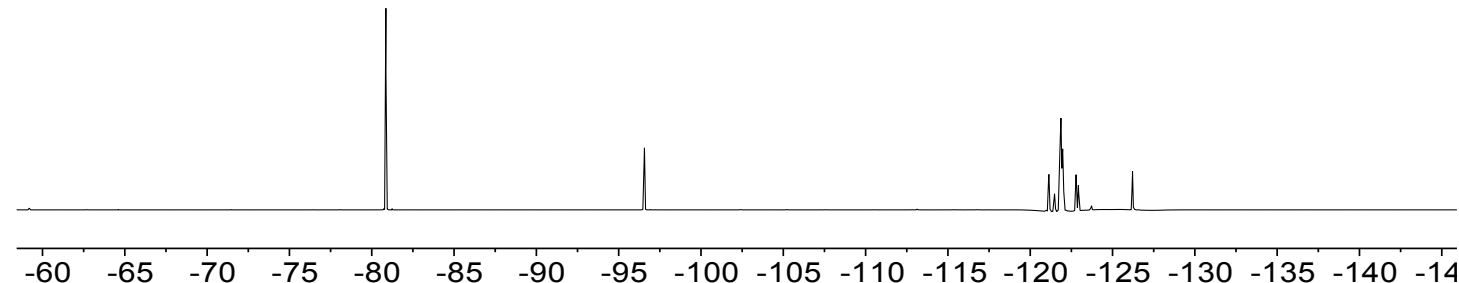


Nom

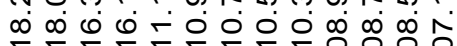

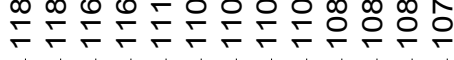

$\infty \wedge$

ํํำ

1 1

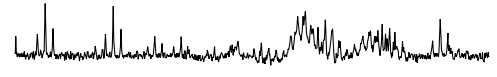
$\begin{array}{lllllll}118 & 116 & 114 & 112 & 110 & 108 & 106\end{array}$

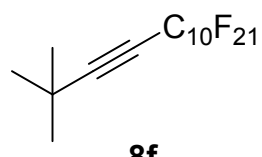

$8 f$

${ }^{13} \mathrm{C}\left\{{ }^{1} \mathrm{H}\right\} \mathrm{NMR}\left(\mathrm{CDCl}_{3}, 151 \mathrm{MHz}\right)$

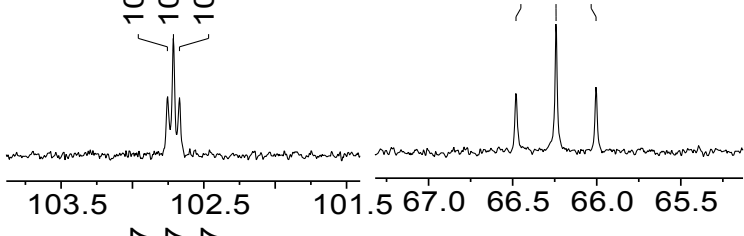

ก.

ลิ

T.T.

$\begin{array}{lll}0 & 0 & 0 \\ 0 & 0 & 0\end{array}$

$\rightarrow$

ลิง

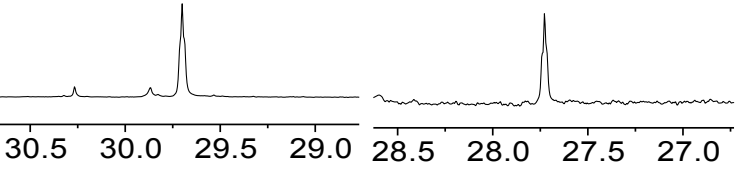

$\begin{array}{lllllllll}30.5 & 30.0 & 29.5 & 29.0 & 28.5 & 28.0 & 27.5 & 27.0\end{array}$

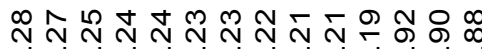

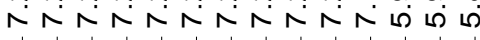

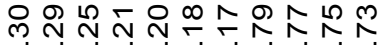

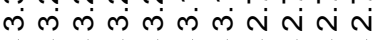<smiles>FC(F)(F)CC1=CCCc2ccccc21</smiles>

11

${ }^{1} \mathrm{H} \mathrm{NMR}\left(\mathrm{CDCl}_{3}, 400 \mathrm{MHz}\right)$

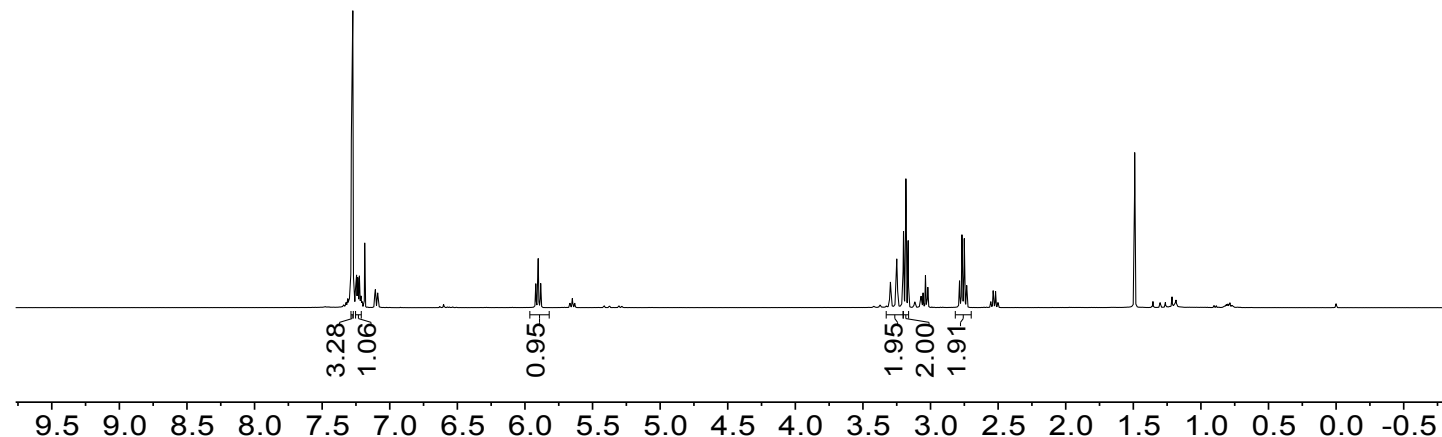



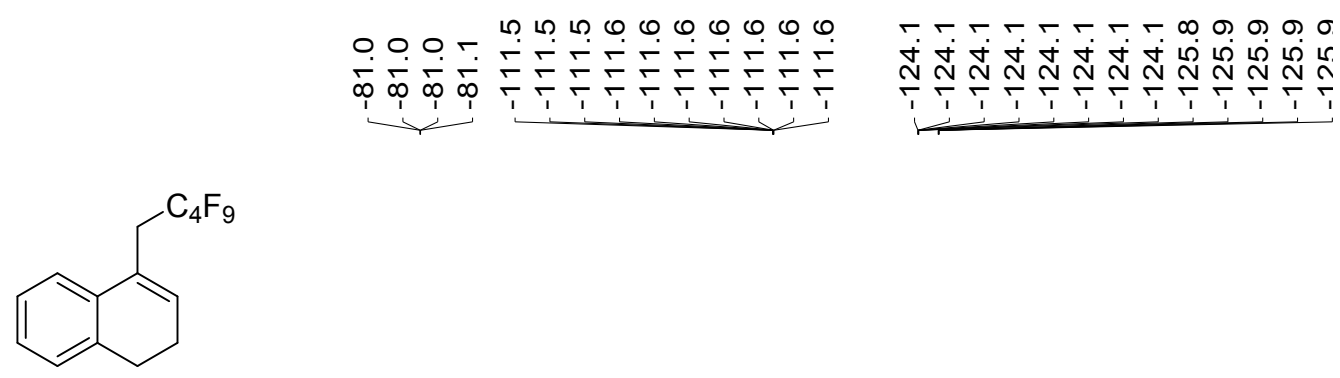

11

${ }^{19} \mathrm{~F} \operatorname{NMR}\left(\mathrm{CDCl}_{3}, 376 \mathrm{MHz}\right)$

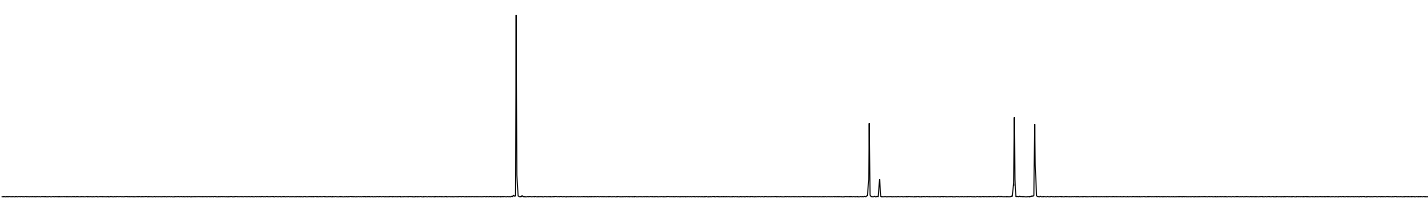

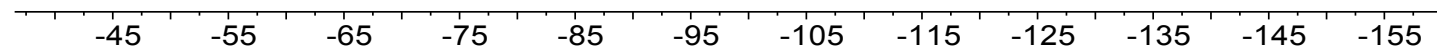




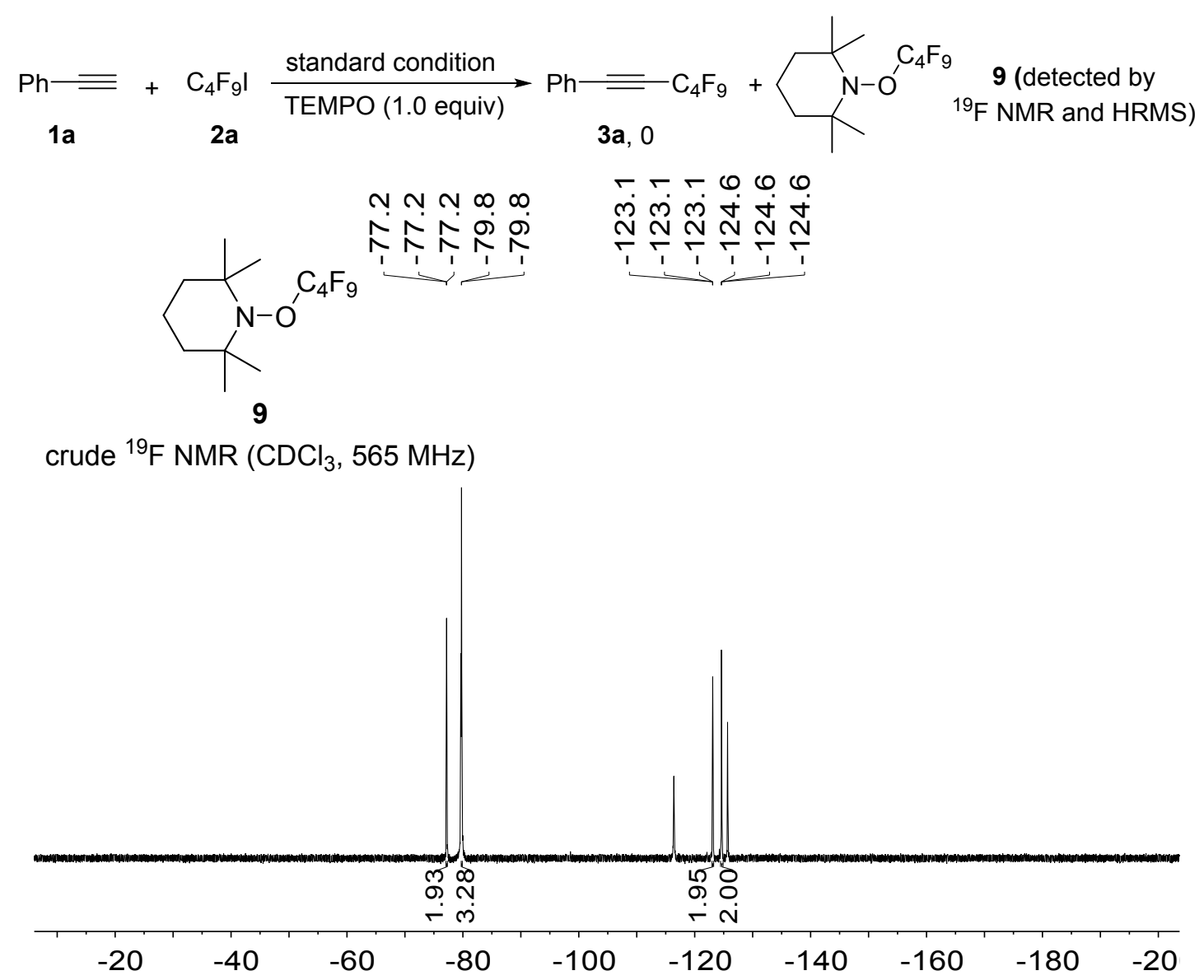

Figure S1. Crude ${ }^{19} \mathrm{~F}$ NMR of TEMPO- $\mathrm{C}_{4} \mathrm{~F}_{9}$ adduct. 

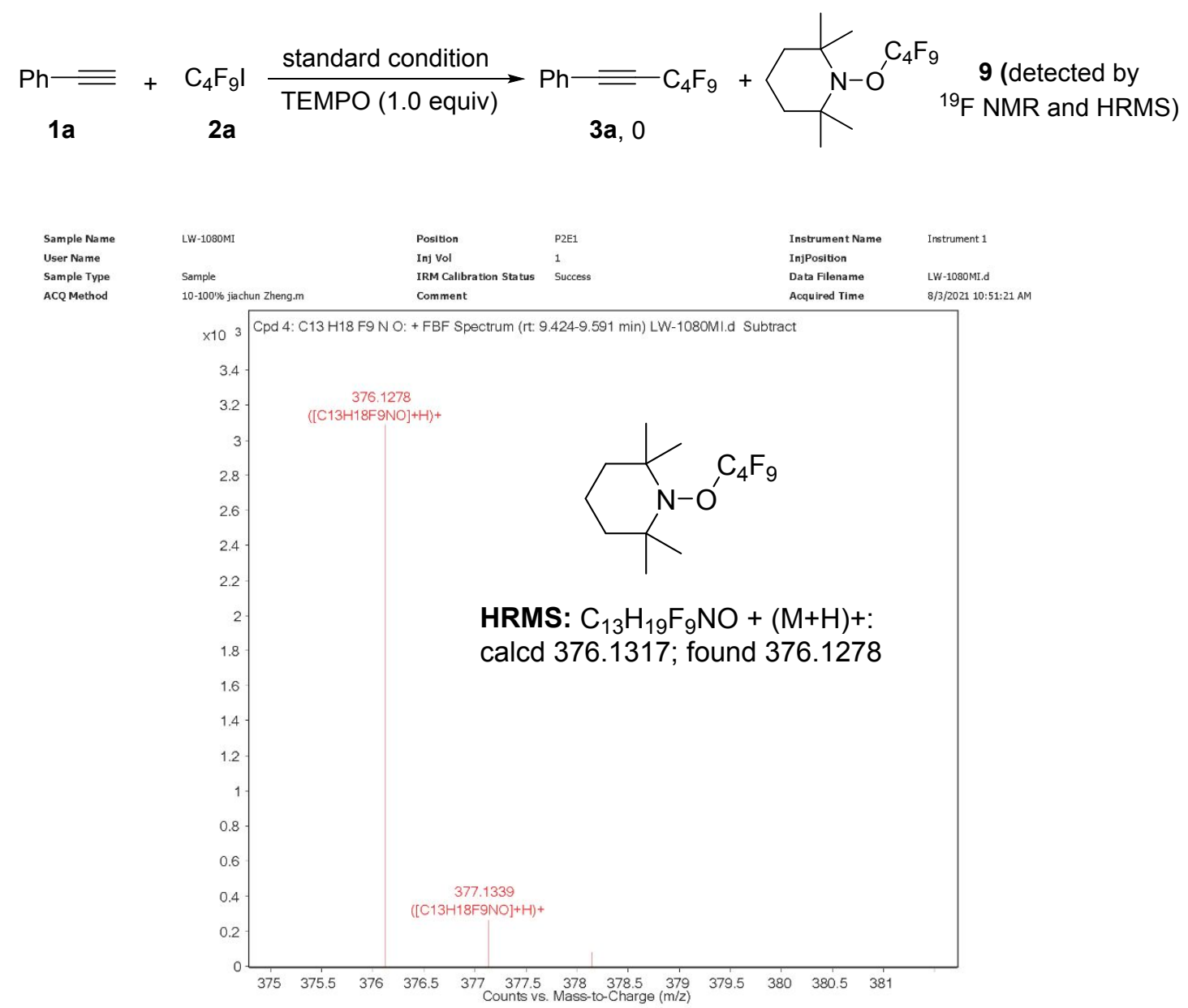

Figure S2. HRMS of TEMPO-C $\mathbf{C}_{4} \mathrm{~F}_{9} 9$ adduct. HRMS of the crude reaction of $1 \mathbf{a}, \mathbf{2 a}$ and TEMPO under optimal reaction conditions (top), showing a peak corresponding to $[9+\mathrm{H}]^{+}$. 